\title{
THE COHOMOLOGY RINGS OF REGULAR NILPOTENT HESSENBERG VARIETIES IN LIE TYPE A
}

\author{
HIRAKU ABE, MEGUMI HARADA, TATSUYA HORIGUCHI, AND MIKIYA MASUDA
}

\begin{abstract}
Let $n$ be a fixed positive integer and $h:\{1,2, \ldots, n\} \rightarrow\{1,2, \ldots, n\}$ a Hessenberg function. The main results of this paper are twofold. First, we give a systematic method, depending in a simple manner on the Hessenberg function $h$, for producing an explicit presentation by generators and relations of the cohomology ring $H^{*}(\operatorname{Hess}(\mathrm{N}, h))$ with $\mathbb{Q}$ coefficients of the corresponding regular nilpotent Hessenberg variety $\operatorname{Hess}(\mathrm{N}, h)$. Our result generalizes known results in special cases such as the Peterson variety and also allows us to answer a question posed by Mbirika and Tymoczko. Moreover, our list of generators in fact forms a regular sequence, allowing us to use techniques from commutative algebra in our arguments. Our second main result gives an isomorphism between the cohomology ring $H^{*}(\operatorname{Hess}(\mathrm{N}, h))$ of the regular nilpotent Hessenberg variety and the $\mathfrak{S}_{n}$-invariant subring $H^{*}(\operatorname{Hess}(\mathrm{S}, h))^{\mathfrak{S}_{n}}$ of the cohomology ring of the regular semisimple Hessenberg variety (with respect to the $\mathfrak{S}_{n}$-action on $H^{*}(\operatorname{Hess}(\mathrm{S}, h)$ ) defined by Tymoczko). Our second main result implies that $\operatorname{dim}_{\mathbb{Q}} H^{k}(\operatorname{Hess}(\mathrm{N}, h))=\operatorname{dim}_{\mathbb{Q}} H^{k}(\operatorname{Hess}(\mathrm{S}, h))^{\mathfrak{S}_{n}}$ for all $k$ and hence partially proves the Shareshian-Wachs conjecture in combinatorics, which is in turn related to the well-known Stanley-Stembridge conjecture. A proof of the full Shareshian-Wachs conjecture was recently given by Brosnan and Chow, but in our special case, our methods yield a stronger result (i.e. an isomorphism of rings) by more elementary considerations. This paper provides detailed proofs of results we recorded previously in a research announcement.
\end{abstract}

\section{Introduction and statement of main results (Theorem A and Theorem B)}

Hessenberg varieties in type A are subvarieties of the full flag variety Flag $\left(\mathbb{C}^{n}\right)$ of nested sequences of linear subspaces in $\mathbb{C}^{n}$. Their geometry and (equivariant) topology have been studied extensively since the late 1980s 10, 12, 11. This subject lies at the intersection of, and makes connections between, many research areas such as geometric representation theory (see for example [43, 18]), combinatorics (see e.g. [16, 33]), and algebraic geometry and topology (see e.g. 31, 7, 48, 28, 36, 37]). A special case of Hessenberg varieties called the Peterson variety $\mathrm{Pet}_{n}$ arises in the study of the quantum cohomology of the flag variety [31, 39, and more generally, geometric properties and invariants of many different types of Hessenberg varieties (including in Lie types other than A) have been widely studied. The (equivariant and ordinary) cohomology rings of Hessenberg varieties have received particular attention. To cite just two examples, the second author and Tymoczko gave an explicit set of generators for $H^{*}\left(P e t_{n}\right)$ and prove a Schubert-calculus-type "Monk formula", resulting in a presentation of $H^{*}\left(\right.$ Pet $\left._{n}\right)$ via generators and relations in 22, and in a different direction, Brion and Carrell showed an isomorphism between the equivariant cohomology ring of a regular nilpotent Hessenberg variety with the affine coordinate ring of a certain affine curve [7. Beyond the two manuscripts just mentioned, there has also been extensive work on the equivariant and ordinary cohomology rings of Springer varieties 9, 46, 13, 25, 2, and of some types of regular nilpotent Hessenberg varieties (including Peterson varieties in different Lie types) [5, 15, 21]. However, it has been an open question to give a general and systematic description of the equivariant cohomology rings of all regular nilpotent Hessenberg varieties [27, Introduction, page 2], to which our results provides an answer (in Lie type A).

In addition, very recent developments provide further evidence that Hessenberg varieties occupy a central place in the fruitful intersection of algebraic geometry, combinatorics, and geometric representation theory. We first recall some background. The well-known Stanley-Stembridge conjecture in combinatorics states that the chromatic symmetric function of the incomparability graph of a so-called $(3+1)$-free poset is $e$ positive. In related work, Stanley [4] also showed a relation between $q$-Eulerian polynomials and a certain

Date: June 19, 2018.

2000 Mathematics Subject Classification. Primary: 55N91, Secondary: 14 N15.

Key words and phrases. Hessenberg varieties, flag varieties, cohomology, regular sequences, Hilbert series, Shareshian-Wachs conjecture. 
$\mathfrak{S}_{n}$-representation on the cohomology of the toric variety associated with the Coxeter complex of type $A_{n-1}$ studied by Procesi [38. The above toric variety is a special case of a regular semisimple Hessenberg variety of type A [11, and Tymoczko [49] has defined $\mathfrak{S}_{n}$-representations on their cohomology rings which generalize the $\mathfrak{S}_{n}$-representation studied by Procesi. Motivated by the above, Shareshian and Wachs formulated in 2011 a conjecture 40 relating the chromatic quasisymmetric function of the incomparability graph of a natural unit interval order and Tymoczko's $\mathfrak{S}_{n}$-representation on the cohomology of the associated regular semisimple Hessenberg variety. While the Shareshian-Wachs conjecture does not imply the StanleyStembridge conjecture, it nevertheless represents a significant step towards its solution. In a 2015 preprint, Brosnan and Chow [8] prove the Shareshian-Wachs conjecture by showing a remarkable relationship between the Betti numbers of different Hessenberg varieties; a key ingredient in their approach is a certain family of Hessenberg varieties, the (cohomology of the) fibers of which are related via monodromy. Our second main result (Theorem B) also contributes to this discussion, as we explain below.

We now describe the two main results (Theorem A and Theorem B below) of this manuscript in more detail. Recall that the flag variety $\operatorname{Flag}\left(\mathbb{C}^{n}\right)$ consists of nested sequences of linear subspaces of $\mathbb{C}^{n}$,

$$
\operatorname{Flag}\left(\mathbb{C}^{n}\right):=\left\{V_{\bullet}=\left(\{0\} \subset V_{1} \subset V_{2} \subset \cdots V_{n-1} \subset V_{n}=\mathbb{C}^{n}\right) \mid \operatorname{dim}_{\mathbb{C}}\left(V_{i}\right)=i \text { for all } i=1, \ldots, n\right\} .
$$

Additionally, let $h:\{1,2, \ldots, n\} \rightarrow\{1,2, \ldots, n\}$ be a Hessenberg function, i.e. $h$ satisfies $h(i) \geq i$ for all $i$ and $h(i+1) \geq h(i)$ for all $i<n$. Also let $\mathbf{N}$ denote a regular nilpotent matrix in $\mathfrak{g l}(n, \mathbb{C})$, i.e. a matrix whose Jordan form consists of exactly one Jordan block with corresponding eigenvalue equal to 0 . Then we may define the regular nilpotent Hessenberg variety (associated to $h$ ) to be the subvariety of Flag $\left(\mathbb{C}^{n}\right.$ ) defined by

$$
\operatorname{Hess}(\mathrm{N}, h):=\left\{V_{\bullet} \in \operatorname{Flag}\left(\mathbb{C}^{n}\right) \mid \mathrm{N} V_{i} \subset V_{h(i)} \text { for all } i=1, \ldots, n\right\} \subset \operatorname{Flag}\left(\mathbb{C}^{n}\right) .
$$

Our first main theorem gives an explicit presentation via generators and relations of the cohomology 1 ring $H^{*}(\operatorname{Hess}(\mathrm{N}, h))$ of the regular nilpotent Hessenberg variety associated to any Hessenberg function $h$. For any pair $i, j$ with $i \geq j$, let $\breve{f}_{i, j}$ be the polynomial

$$
\check{f}_{i, j}:=\sum_{k=1}^{j}\left(x_{k} \prod_{\ell=j+1}^{i}\left(x_{k}-x_{\ell}\right)\right) \text { for } i \geq j
$$

with the convention $\prod_{\ell=j+1}^{j}\left(x_{k}-x_{\ell}\right)=1$.

Theorem A. Let $n$ be a positive integer and $h:\{1,2, \ldots, n\} \rightarrow\{1,2, \ldots, n\}$ a Hessenberg function. Let $\mathbf{N}$ denote a regular nilpotent matrix in $\mathfrak{g l}(n, \mathbb{C})$ and let $\operatorname{Hess}(\mathrm{N}, h) \subset$ Flag $\left(\mathbb{C}^{n}\right)$ be the associated regular nilpotent Hessenberg variety. Then the restriction map

$$
H^{*}\left(\operatorname{Flag}\left(\mathbb{C}^{n}\right)\right) \rightarrow H^{*}(\operatorname{Hess}(\mathrm{N}, h))
$$

is surjective, and there is an isomorphism of graded $\mathbb{Q}$-algebras

$$
H^{*}(\operatorname{Hess}(\mathrm{N}, h)) \cong \mathbb{Q}\left[x_{1}, \ldots, x_{n}\right] / \check{I_{h}}
$$

where $\check{I}_{h}$ is the ideal of $\mathbb{Q}\left[x_{1}, \ldots, x_{n}\right]$ defined by

$$
\check{I}_{h}:=\left(\check{f}_{h(j), j} \mid 1 \leq j \leq n\right) .
$$

The following points are worth noting immediately. Firstly, the equation (1.2) gives a simple closed formula for the polynomials $\breve{f}_{h(j), j}$ generating the ideal $\check{I}_{h}$ in (1.3); moreover, the ideal depends in a manifestly simple and systematic manner on the Hessenberg function $h$. Secondly, these generators $\left\{\check{f}_{h(j), j}\right\}_{j=1}^{n}$ have algebraic properties which make them particularly useful. Specifically, the $\check{f}_{h(j), j}$ (as well as their equivariant counterparts $f_{i, j}$ which we discuss below) in fact form a regular sequence (cf. Definition 6.1) in the sense of commutative algebra, and it is precisely this property which allows us to exploit techniques in e.g. the theory of Hilbert series and Poincaré duality algebras to prove both of our main results. Thirdly, we can answer a question posed by Mbirika and Tymoczko [34, Question 2]: they asked whether $H^{*}(\operatorname{Hess}(\mathrm{N}, h))$ is isomorphic to the quotient of $\mathbb{Q}\left[x_{1}, \ldots, x_{n}\right]$ by a certain ideal, described in detail in [34, which is generated by "truncated symmetric polynomials". Our Theorem A says that, in general, the answer is "No". For

${ }^{1}$ Throughout this document (unless explicitly stated otherwise) we work with cohomology with coefficients in $\mathbb{Q}$. 
instance, in the special case of the Peterson variety Pet $_{n}$ of complex dimension $n$ for $n \geq 3$, it is not difficult to see directly from Mbirika and Tymoczko's definitions in 34 that their ring contains a non-zero element of degree 2 whose square is equal to 0 , whereas one can see from our presentation (1.3) that $H^{*}\left(\right.$ Pet $\left._{n}\right)$ contains no such element. Finally, our Theorem A generalizes known results: in the special cases of the full flag variety Flag $\left(\mathbb{C}^{n}\right)$ and the Peterson variety $\mathrm{Pet}_{n}$, the presentation given in Theorem A recovers previously known presentations of the relevant cohomology rings (cf. Remarks 3.4).

Next we turn to Theorem B, for which we need additional terminology. Let $h$ be a Hessenberg function and this time let $\mathbf{S}$ denote a regular semisimple matrix in $\mathfrak{g l}(n, \mathbb{C})$, i.e. a matrix which is diagonalizable with distinct eigenvalues. Then the regular semisimple Hessenberg variety (associated to $h$ ) is defined to be

$$
\operatorname{Hess}(\mathrm{S}, h):=\left\{V_{\bullet} \in \operatorname{Flag}\left(\mathbb{C}^{n}\right) \mid \mathrm{S} V_{i} \subset V_{h(i)} \text { for all } i=1, \ldots, n\right\} \subset \operatorname{Flag}\left(\mathbb{C}^{n}\right) .
$$

The cohomology rings of these varieties admit an action of the symmetric group $\mathfrak{S}_{n}$, as Tymoczko pointed out many years ago 49. In Theorem B, we prove - for a fixed Hessenberg function $h$ - that there exists an isomorphism of graded rings between the cohomology ring of the corresponding regular nilpotent Hessenberg variety and the $\mathfrak{S}_{n}$-invariant subring of the cohomology ring of the corresponding regular semisimple Hessenberg variety. More precisely, we have the following.

Theorem B. Let $n$ be a positive integer and $h:\{1,2, \ldots, n\} \rightarrow\{1,2, \ldots, n\}$ a Hessenberg function. Let $\mathrm{N}$ denote a regular nilpotent matrix and $\mathrm{S}$ denote a regular semisimple matrix in $\mathfrak{g l}(n, \mathbb{C})$. Let $\operatorname{Hess}(\mathrm{N}, h)$ and $\operatorname{Hess}(\mathrm{S}, h)$ be the associated regular nilpotent and regular semisimple Hessenberg varieties respectively. Then there exists a unique graded $\mathbb{Q}$-algebra homomorphism $\mathcal{A}: H^{*}(\operatorname{Hess}(\mathrm{N}, h)) \rightarrow H^{*}(\operatorname{Hess}(\mathrm{S}, h))$ making the following diagram commute:

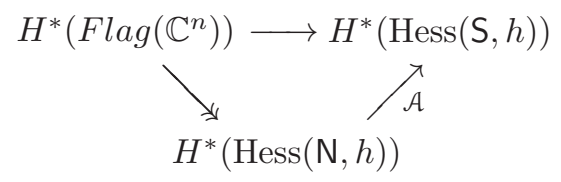

where the maps $H^{*}\left(F \operatorname{lag}\left(\mathbb{C}^{n}\right)\right) \rightarrow H^{*}(\operatorname{Hess}(\mathrm{S}, h))$ and $H^{*}\left(F \operatorname{lag}\left(\mathbb{C}^{n}\right)\right) \rightarrow H^{*}(\operatorname{Hess}(\mathrm{N}, h))$ are induced from the inclusions $\operatorname{Hess}(\mathrm{S}, h) \hookrightarrow$ Flag $\left(\mathbb{C}^{n}\right)$ and $\operatorname{Hess}(\mathrm{N}, h) \hookrightarrow$ Flag $\left(\mathbb{C}^{n}\right)$ respectively. Moreover, the image of $\mathcal{A}$ is precisely the ring $H^{*}(\operatorname{Hess}(\mathrm{S}, h))^{\mathfrak{S}_{n}}$ of $\mathfrak{S}_{n}$-invariants in $H^{*}(\operatorname{Hess}(\mathrm{S}, h))$, and when the target of $\mathcal{A}$ is restricted to this invariant subring, then

$$
\mathcal{A}: H^{*}(\operatorname{Hess}(\mathrm{N}, h)) \rightarrow H^{*}(\operatorname{Hess}(\mathrm{S}, h))^{\mathfrak{S}_{n}}
$$

is an isomorphism of graded $\mathbb{Q}$-algebras.

As a special case, we note that Theorem B implies that the cohomology $H^{*}\left(\right.$ Pet $\left._{n}\right)$ of the Peterson variety Pet $t_{n}$ is isomorphic to the $\mathfrak{S}_{n}$-invariant subring $H^{*}(X)^{\mathfrak{S}_{n}}$ of the cohomology ring of the toric variety $X$ associated with the Coxeter complex of type $\mathrm{A}_{n-1}$. This fact could be previously seen by comparing the description of $H^{*}\left(\right.$ Pet $\left._{n}\right)$ given explicitly in [15] to the description of $H^{*}(X)^{\mathfrak{S}_{n}}$ stated without proof in [29]. Indeed, the striking similarity of the rings in [29] and [15] was our original motivation to prove Theorem B.

Next we discuss the relationship between Theorem B and recent research in combinatorics. As briefly discussed above, Shareshian and Wachs conjectured a precise relationship between the (Frobenius characteristic of) Tymoczko's $\mathfrak{S}_{n}$-representation on the cohomology group of a regular semisimple Hessenberg variety Hess $(\mathrm{S}, h)$ and the chromatic quasisymmetric function $X_{G}(x, t)$ of a graph $G$ defined from the Hessenberg function $h$ [40, 41]. (Details are in Section 11]) When both sides of their conjectured equality are expanded in terms of Schur functions $s_{\lambda}(x)$ for $\lambda$ a partition of $n$, their conjecture can be interpreted as a set of equalities of the coefficients (which are polynomials in $t$ ) of $s_{\lambda}(x)$ on each side. In [41, Theorem 6.9] Shareshian and Wachs also obtain a closed formula for the coefficient of $s_{n}(x)$, i.e. the coefficient corresponding to the trivial representation, and it agrees with the Hilbert series (also a polynomial in $t$ ) of $H^{*}(\operatorname{Hess}(\mathrm{N}, h)$ ). Upon unraveling some definitions, it readily follows that our Theorem B proves the Shareshian-Wachs conjecture for the coefficient of the trivial representation. During the preparation of this manuscript we learned that Brosnan and Chow have independently proved the full Shareshian-Wachs conjecture, i.e. the equality of the coefficients for all Schur functions, not just $s_{n}(x)$. However, we note that while Brosnan and Chow obtain 
an equality of dimensions of vectors spaces $\operatorname{dim} H^{k}(\operatorname{Hess}(\mathrm{N}, h))=\operatorname{dim} H^{k}(\operatorname{Hess}(\mathrm{S}, h))^{\mathfrak{S}_{n}}$ for varying $k$, Theorem 76], their techniques do not appear to immediately yield further information about the product structure on the rings $H^{*}(\operatorname{Hess}(\mathrm{N}, h))$ and $H^{*}(\operatorname{Hess}(\mathrm{S}, h))^{\mathfrak{S}_{n}}$. Thus, for our special case, our Theorem B is stronger than the corresponding result in [8]. Moreover, while Brosnan and Chow's arguments utilize deep and powerful results in the theory of local systems and perverse sheaves (specifically, the local invariant cycle theorem of Beilinson-Bernstein-Deligne), our methods are more elementary, thus providing a useful alternative perspective on this circle of ideas.

We now briefly discuss the methods used in the proofs of Theorems A and B. Our basic strategy is to exploit the presence of torus actions on the varieties in question and to use well-known techniques in equivariant topology, e.g. localization and Goresky-Kottwitz-MacPherson (GKM) theory. More specifically, we state and prove an "equivariant" version of Theorem A using certain polynomials $f_{h(j), j}$ which lift in an appropriate sense the polynomials $\check{f}_{h(j), j}$ appearing in Theorem A. For Theorem B, we use GKM theory to combinatorially describe both the equivariant cohomology of $\operatorname{Hess}(\mathrm{S}, h)$ and the $\mathfrak{S}_{n}$-action on it, and also use some standard commutative algebra results on Poincaré duality algebras together with the fact that the polynomials $\left\{\check{f}_{h(j), j}\right\}$ form a regular sequence to obtain our result.

We take a moment to record an open question motivated by our work. It would be of interest to determine the ring structure of the full cohomology ring $H^{*}(\operatorname{Hess}(\mathrm{S}, h))$ (i.e. not just the $\mathfrak{S}_{n}$-invariant subring) of regular semisimple Hessenberg varieties for arbitrary Hessenberg functions $h$. We have preliminary results in this direction. For example, it turns out that, when $h=(h(1), n, \ldots, n)$ with an arbitrary value of $h(1)$, the classes $g_{1, i}$ and $\hat{\tau}_{i}$ (see Section 10 for the precise definitions) for $i=1, \ldots, n$ generate $H^{*}(\operatorname{Hess}(\mathrm{S}, h))$ as a ring, and it is possible to give an explicit presentation of $H^{*}(\operatorname{Hess}(\mathrm{S}, h))$ with respect to these generators. Similarly, the corresponding classes also can be shown to generate the ring $H^{*}(\operatorname{Hess}(\mathrm{S}, h))$ for the case $h=(m, \ldots, m, n, \ldots, n)$ for any $m$. Furthermore, we have found a finite list of generators of $H^{*}(\operatorname{Hess}(\mathrm{S}, h))$ for the case $h=(h(1), h(2), n, \ldots, n)$ with arbitrary values of $h(1)$ and $h(2)$. In an ongoing project, we are investigating the problem of finding ring generators of $H^{*}(\operatorname{Hess}(\mathrm{S}, h))$ for arbitrary Hessenberg functions $h$ which behave well with respect to Tymoczko's $\mathfrak{S}_{n}$-representation.

The paper is organized as follows. After briefly reviewing some background and terminology on regular nilpotent Hessenberg varieties in Section 2, we state the equivariant version of our Theorem A in Section 3 as Theorem 3.3 The key properties of the polynomials $f_{i, j}$, necessary for the proof of Theorem 3.3, are recorded in Section 4. The fact that the equivariant version of the homomorphism (1.3) is well-defined is shown in Section 5. To prove that it is in fact an isomorphism requires some preparatory arguments using Hilbert series, which are recorded in Section 6. In Section 7 using the results of the previous sections we are able to complete the proof of Theorem 3.3 and hence also of Theorem A. Next, turning our attention to Theorem $\mathrm{B}$, we quickly recount some background and terminology concerning regular semisimple Hessenberg varieties in Section 8. We recall and also prove some essential facts about Tymoczko's $\mathfrak{S}_{n}$-action on the (equivariant and ordinary) cohomology of regular semisimple Hessenberg varieties in Section 9. We prove Theorem B in Section 10 and discuss the connection between our results and the Shareshian-Wachs conjecture in Section 11.

Acknowledgements. We are grateful to Satoshi Murai for his invaluable help in the commutative algebra arguments. We also thank John Shareshian and Michelle Wachs for their kind support and their interest in this project. We are also grateful to Takashi Sato for improving the proof of Lemma 10.2. The first author was supported in part by the JSPS Program for Advancing Strategic International Networks to Accelerate the Circulation of Talented Researchers: "Mathematical Science of Symmetry, Topology and Moduli, Evolution of International Research Network based on OCAMI", and he is grateful to Yoshihiro Ohnita for enhancing his research projects. He was also supported by a JSPS Grant-in-Aid for Young Scientists (B): 15K17544. The second author was partially supported by an NSERC Discovery Grant, a Canada Research Chair (Tier 2) Award, an Association for Women in Mathematics Ruth Michler Award, and a Japan Society for the Promotion of Science Invitation Fellowship for Research in Japan (Fellowship ID L-13517). The third author was partially supported by a JSPS Grant-in-Aid for JSPS Fellows: 15J09343. The fourth author was partially supported by a JSPS Grant-in-Aid for Scientific Research 25400095. 


\section{BACKGROUND AND PRELIMINARIES}

In this section we recall some background and establish some terminology for the rest of the paper. Specifically, in Section 2.1 we recall the definitions of the regular nilpotent Hessenberg varieties, as well as the torus actions on them. We also quickly recount some techniques in torus-equivariant cohomology which will be used throughout. In Section 2.2 we analyze the torus-fixed point set of the regular nilpotent Hessenberg variety which plays a key role in our later arguments.

2.1. The setup. Hessenberg varieties in Lie type A are subvarieties of the (full) flag variety $F$ lag $\left(\mathbb{C}^{n}\right)$, which is the collection of sequences of nested linear subspaces of $\mathbb{C}^{n}$ :

$$
\operatorname{Flag}\left(\mathbb{C}^{n}\right):=\left\{V_{\bullet}=\left(\{0\} \subset V_{1} \subset V_{2} \subset \cdots V_{n-1} \subset V_{n}=\mathbb{C}^{n}\right) \mid \operatorname{dim}_{\mathbb{C}}\left(V_{i}\right)=i \text { for all } i=1, \ldots, n\right\} .
$$

It is well-known that $\operatorname{Flag}\left(\mathbb{C}^{n}\right)$ can also be realized as a homogeneous space $\operatorname{GL}(n, \mathbb{C}) / B$ where $B$ is the standard Borel subgroup of upper-triangular invertible matrices. Thus there is a natural action of $\mathrm{GL}(n, \mathbb{C})$ on Flag $\left(\mathbb{C}^{n}\right)$ given by left multiplication on cosets.

A Hessenberg variety in Flag $\left(\mathbb{C}^{n}\right)$ is specified by two pieces of data: a Hessenberg function and a choice of an element in the Lie algebra $\mathfrak{g l}(n, \mathbb{C})$ of $\mathrm{GL}(n, \mathbb{C})$. We begin by discussing the first of these parameters. Throughout this document we use the notation

$$
[n]:=\{1,2, \ldots, n\}
$$

Definition 2.1. A Hessenberg function is a function $h:[n] \rightarrow[n]$ satisfying the following two conditions

$$
\begin{array}{ll}
h(i) \geq i & \text { for } i \in[n], \\
h(i+1) \geq h(i) & \text { for } i \in[n-1] .
\end{array}
$$

We frequently write a Hessenberg function by listing its values in sequence, i.e. $h=(h(1), h(2), \ldots, h(n))$.

We also define $H_{n}$ to be the set of Hessenberg functions $h:[n] \rightarrow[n]$, i.e.

$$
H_{n}:=\{h:[n] \rightarrow[n] \mid h \text { is a Hessenberg function }\} \text {. }
$$

For the discussion to follow, it will be useful to introduce some terminology associated to a given Hessenberg function.

Definition 2.2. Let $h \in H_{n}$ be a Hessenberg function. Then we define the Hessenberg subspace $H(h)$ to be the linear subspace of $\mathfrak{g l}(n, \mathbb{C}) \cong \operatorname{Mat}(n \times n, \mathbb{C})$ specified as follows:

$$
H(h):=\left\{A=\left(a_{i j}\right)_{i, j \in[n]} \in \mathfrak{g l}(n, \mathbb{C}) \mid a_{i j}=0 \text { if } i>h(j)\right\} .
$$

It is important to note that the $H(h)$ is frequently not a Lie subalgebra of $\mathfrak{g l}(n, \mathbb{C})$. However, it is stable under the conjugation action of the usual maximal torus $T$ (of invertible diagonal matrices), and we may decompose $H(h)$ into eigenspaces with respect to this action as

$$
H(h) \cong \mathfrak{b} \oplus\left(\bigoplus_{\substack{i, j \in[n], j<i \leq h(j)}} \mathfrak{g l}(n, \mathbb{C})_{(i, j)}\right)
$$

where $\mathfrak{b}=\operatorname{Lie}(B)$ denotes the Lie algebra of the Borel subgroup of upper-triangular matrices, and $\mathfrak{g l}(n, \mathbb{C})_{(i, j)}$ denotes the 1 -dimensional $T$-weight space of $\mathfrak{g l}(n, \mathbb{C})$ spanned by the elementary matrix $E_{i, j}$ with a 1 in the $(i, j)$-th entry and 0's elsewhere. In Lie-theoretic language, the $(i, j)$ satisfying the condition in the RHS of (2.3) correspond to the negative roots of $\mathfrak{g l}(n, \mathbb{C})$ whose corresponding root spaces appear in $H(h)$. It will be useful later on to focus attention on these roots, so we introduce the notation

$$
\mathrm{NR}(h):=\{(i, j) \in[n] \times[n] \mid j<i \leq h(j)\} .
$$

It is conceptually useful to express $H(h)$ pictorially by drawing a configuration of boxes on a square grid of size $n \times n$ whose shaded boxes correspond to the roots appearing in (2.3), and from this perspective, the set $\mathrm{NR}(h)$ corresponds one-to-one with "the boxes in (the picture associated to) $H(h)$ which lie strictly below the main diagonal". See Figure 1 

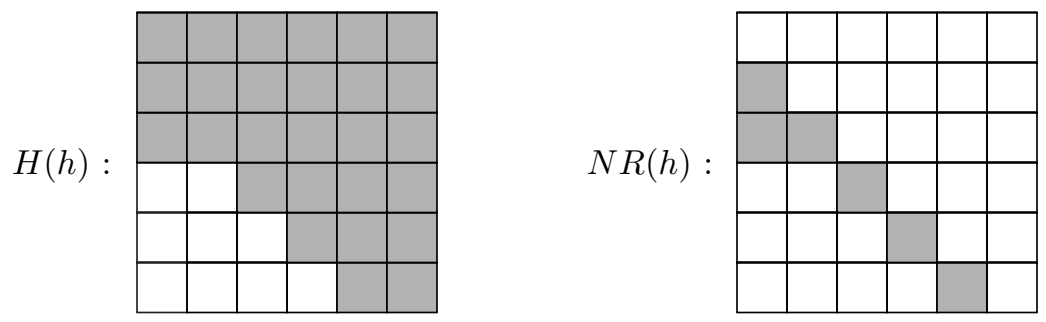

Figure 1. The pictures of $H(h)$ and $\mathrm{NR}(h)$ for $h=(3,3,4,5,6,6)$.

We now introduce the main geometric objects of interest in this manuscript. Let $h:[n] \rightarrow[n]$ be a Hessenberg function and let $A$ be an $n \times n$ matrix in $\mathfrak{g l}(n, \mathbb{C})$. Then the Hessenberg variety Hess $(A, h)$ associated to $h$ and $A$ is defined to be

$$
\operatorname{Hess}(A, h):=\left\{V_{\bullet} \in \operatorname{Flag}\left(\mathbb{C}^{n}\right) \mid A V_{i} \subset V_{h(i)} \text { for all } i \in[n]\right\} \subset \operatorname{Flag}\left(\mathbb{C}^{n}\right)
$$

In particular, by definition $\operatorname{Hess}(A, h)$ is a subvariety of $F \operatorname{lag}\left(\mathbb{C}^{n}\right)$, and if $h=(n, n, \ldots, n)$, then it is immediate from (2.5) that Hess $(A, h)=F \operatorname{lag}\left(\mathbb{C}^{n}\right)$ for any choice of $A$. Thus the full flag variety Flag $\left(\mathbb{C}^{n}\right)$ is itself a special case of a Hessenberg variety; this will be important later on. We also remark that if $g \in \operatorname{GL}(n, \mathbb{C})$, then $\operatorname{Hess}(A, h)$ and $\operatorname{Hess}\left(g A g^{-1}, h\right)$ can be identified via the action of $\operatorname{GL}(n, \mathbb{C})$ on $\operatorname{Flag}\left(\mathbb{C}^{n}\right)$. In particular, important geometric features of Hessenberg varieties are frequently dependent only on the conjugacy class of the element $A \in \mathfrak{g l}(n, \mathbb{C})$, and not on $A$ itself.

In this paper we focus on two special cases of Hessenberg varieties, as we now describe. Let $\mathrm{N}$ denote a regular nilpotent matrix in $\mathfrak{g l}(n, \mathbb{C})$, i.e. a matrix whose Jordan form consists of exactly one Jordan block with corresponding eigenvalue equal to 0 . Similarly let $\mathbf{S}$ denote a regular semisimple matrix in $\mathfrak{g l}(n, \mathbb{C})$, i.e. a matrix which is diagonalizable with distinct eigenvalues. Then, for any choice of Hessenberg function $h \in H_{n}$, we call $\operatorname{Hess}(\mathrm{N}, h)$ the regular nilpotent Hessenberg variety (associated to $h$ ) and call Hess $(\mathrm{S}, h)$ the regular semisimple Hessenberg variety (associated to $h$ ). Both of the above types of Hessenberg varieties have been much studied, and it is known, for example, that Hess $(\mathrm{N}, h)$ is irreducible [4] and possibly singular [31, 28, while $\operatorname{Hess}(\mathrm{S}, h)$ is smooth, and possibly non-connected [11. As already noted, the essential geometry of the regular semisimple Hessenberg variety Hess $(\mathrm{S}, h)$ depends only on the conjugacy class of S. In fact, even more is true: it can be seen, for instance, that the (ordinary or equivariant) cohomology of $\operatorname{Hess}(\mathrm{S}, h)$ is also independent of the choices of the (distinct) eigenvalues of $\mathrm{S}$ (see e.g. [49]). For concreteness, henceforth we will always assume that $\mathrm{N}$ and $\mathrm{S}$ are of the form

$$
\mathrm{N}=\left(\begin{array}{ccccc}
0 & 1 & & & \\
& 0 & 1 & & \\
& & \ddots & \ddots & \\
& & & 0 & 1 \\
& & & & 0
\end{array}\right) \quad \text { and } \quad \mathrm{S}=\left(\begin{array}{cccc}
\mu_{1} & & & \\
& \mu_{2} & & \\
& & \ddots & \\
& & & \\
& & & \mu_{n}
\end{array}\right)
$$

with respect to the standard basis of $\mathbb{C}^{n}$, where $\mu_{1}, \mu_{2}, \ldots, \mu_{n}$ are mutually distinct complex numbers. We also note that the dimensions of $\operatorname{Hess}(\mathrm{N}, h)$ and $\operatorname{Hess}(\mathrm{S}, h)$ have been computed explicitly in terms of the Hessenberg function [11, 42, and they coincide:

$$
\operatorname{dim}_{\mathbb{C}} \operatorname{Hess}(\mathrm{N}, h)=\operatorname{dim}_{\mathbb{C}} \operatorname{Hess}(\mathrm{S}, h)=\sum_{j=1}^{n}(h(j)-j) .
$$

Note that this number is also the number of boxes in the picture associated to $\mathrm{NR}(h)$. For example, if $h=(3,3,4,5,6,6)$ as in Figure 1, then $\operatorname{dim}_{\mathbb{C}} \operatorname{Hess}(\mathrm{N}, h)=\operatorname{dim}_{\mathbb{C}} \operatorname{Hess}(\mathrm{S}, h)=6$. 
An essential ingredient in our discussion is the presence of torus actions on both $\operatorname{Hess}(\mathrm{N}, h)$ and $\operatorname{Hess}(\mathrm{S}, h)$. In both cases, the actions are induced from one on the ambient variety $F l a g\left(\mathbb{C}^{n}\right)$. Let

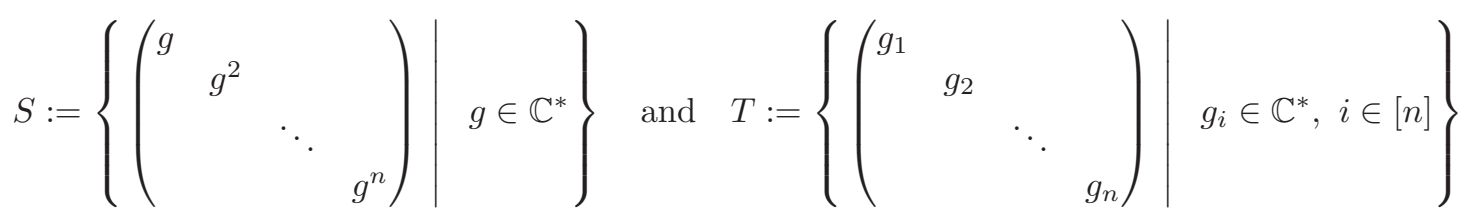

which are subgroup of $\mathrm{GL}(n, \mathbb{C})$ and hence naturally act on $F \operatorname{lag}\left(\mathbb{C}^{n}\right)$. We have $\operatorname{dim}_{\mathbb{C}} S=1$ and $\operatorname{dim}_{\mathbb{C}} T=n$. Notice that $S$ commutes with the matrix N (up to a scalar multiplication) and that $T$ commutes with $\mathrm{S}$. It is straightforward to see that the $S$-action on $\operatorname{Flag}\left(\mathbb{C}^{n}\right)$ preserves $\operatorname{Hess}(\mathrm{N}, h)$ ([23, Lemma 5.1]) and that the $T$-action on Flag $\left(\mathbb{C}^{n}\right)$ preserves Hess $(\mathrm{S}, h)$. However, note that the $T$-action does not preserve Hess $(\mathrm{N}, h)$ in general.

These torus actions lead us to a study of the equivariant cohomology of Hessenberg varieties, so we now quickly recall some basic background on equivariant topology. Suppose $X$ is a topological space which admits a continuous action by the torus $T$. The $T$-equivariant cohomology $H_{T}^{*}(X)$ is defined to be the ordinary cohomology $H^{*}\left(X \times_{T} E T\right)$ where $E T \rightarrow B T$ is the universal principal bundle of $T$. In particular, $H_{T}^{*}(\mathrm{pt})=H^{*}(B T)$ and $H_{T}^{*}(X)$ is an $H_{T}^{*}(\mathrm{pt})$-module. We have

$$
H^{*}(B T) \cong \operatorname{Sym}_{\mathbb{Q}}\left(\operatorname{Hom}\left(T, \mathbb{C}^{*}\right) \otimes_{\mathbb{Z}} \mathbb{Q}\right)
$$

so we may identify $H^{*}(B T)$ with the polynomial ring $\mathbb{Q}\left[t_{1}, \ldots, t_{n}\right]$ where the element $t_{i}$ is the first Chern class of the line bundle over $B T$ corresponding to the projection $T \rightarrow \mathbb{C}^{*}, \operatorname{diag}\left(g_{1}, \ldots, g_{n}\right) \mapsto g_{i}$.

Next we recall some standard constructions on the ambient space Flag $\left(\mathbb{C}^{n}\right)$ leading to a well-known ring presentation for the equivariant cohomology of Flag $\left(\mathbb{C}^{n}\right)$. Let $E_{i}$ denote the $i$-th tautological vector bundle over Flag $\left(\mathbb{C}^{n}\right)$; namely, $E_{i}$ is the sub-bundle of the trivial vector bundle Flag $\left(\mathbb{C}^{n}\right) \times \mathbb{C}^{n}$ over Flag $\left(\mathbb{C}^{n}\right)$ whose fiber over a point $V_{\bullet}=\left(V_{1} \subset \cdots \subset V_{n}\right) \in \operatorname{Flag}\left(\mathbb{C}^{n}\right)$ is exactly $V_{i}$. Let

$$
\tau_{i}^{T} \in H_{T}^{2}\left(\operatorname{Flag}\left(\mathbb{C}^{n}\right)\right)
$$

denote the $T$-equivariant first Chern class of the tautological line bundle $E_{i} / E_{i-1}$. It is known that $H_{T}^{*}\left(F \operatorname{Flag}\left(\mathbb{C}^{n}\right)\right)$ is generated as a ring by the elements $\tau_{1}^{T}, \ldots, \tau_{n}^{T}$ together with the $t_{1}, \ldots, t_{n}$ (the latter coming from the $H_{T}^{*}(\mathrm{pt})$-module structure). Indeed, there is a ring isomorphism

$$
H_{T}^{*}\left(\operatorname{Flag}\left(\mathbb{C}^{n}\right)\right) \cong \mathbb{Q}\left[x_{1}, \ldots, x_{n}, t_{1}, \ldots, t_{n}\right] /\left(e_{i}\left(x_{1}, \ldots, x_{n}\right)-e_{i}\left(t_{1}, \ldots, t_{n}\right) \mid i \in[n]\right)
$$

defined by sending the polynomial ring variables $x_{i}$ on the RHS to the Chern class $\tau_{i}^{T}$ of the $i$-th tautological line bundle and the variables $t_{i}$ to the Chern classes (which by slight abuse of notation we denote by the same) $t_{i}$, and the $e_{i}$ denotes the degree- $i$ elementary symmetric polynomial in the relevant variables. Here and below it should be noted that the degrees of the variables in question are 2, i.e.

$$
\operatorname{deg} x_{i}=\operatorname{deg} t_{i}=2 \text { for all } i \in[n] .
$$

By setting the variables $t_{i}$ equal to 0 , we can also describe the non-equivariant cohomology ring $H^{*}\left(F \operatorname{lag}\left(\mathbb{C}^{n}\right)\right)$ as follows. Let

$$
\tau_{i} \in H^{2}\left(\operatorname{Flag}\left(\mathbb{C}^{n}\right)\right)
$$

be the (non-equivariant) first Chern class of the tautological line bundle $E_{i} / E_{i-1}$. Then we have

$$
H^{*}\left(\operatorname{Flag}\left(\mathbb{C}^{n}\right)\right) \cong \mathbb{Q}\left[x_{1}, \ldots, x_{n}\right] /\left(e_{i}\left(x_{1}, \ldots, x_{n}\right) \mid i \in[n]\right)
$$

where each $x_{i}$ corresponds to the first Chern class $\tau_{i}$.

As mentioned above, we will also analyze the action of the 1-dimensional subgroup $S$ of $T$. Let $\mathbb{C}$ temporarily denote the 1-dimensional representation of $S$ defined by the group homomorphism $\operatorname{diag}\left(g, g^{2}, \ldots, g^{n}\right) \mapsto g$ and consider the associated line bundle $E S \times_{S} \mathbb{C} \rightarrow B S$. Let

$$
t \in H^{2}(B S)
$$

denote the first Chern class of this line bundle. As in the case of $T$ above, we identify $H^{*}(B S)$ with the polynomial ring $\mathbb{Q}[t]$. 
A useful and fundamental technique in torus-equivariant topology is the restriction to the fixed point set of the torus action. If the Serre spectral sequence of the fibration $E T \times_{T} X \rightarrow B T$ collapses at the $E_{2}$-stage, then the equivariant cohomology of $X$ (with $\mathbb{Q}$-coefficients) is a free $H_{T}^{*}(\mathrm{pt})$-module, i.e. as an $H_{T}^{*}(\mathrm{pt})$ module we have $H_{T}^{*}(X) \cong H_{T}^{*}(\mathrm{pt}) \otimes_{\mathbb{Q}} H^{*}(X)$. In addition, under some technical hypotheses on $X$ which are satisfied by the spaces considered in this paper 23 it follows from the localization theorem [26, p.40] that the inclusion $X^{T} \hookrightarrow X$ of the $T$-fixed point set induces an injection $H_{T}^{*}(X) \hookrightarrow H_{T}^{*}\left(X^{T}\right)$. Any Hessenberg variety (in Lie type A) admits a paving by complex affines [48, Theorem 7.1], so their cohomology rings are concentrated in even degrees. Hence the corresponding Serre spectral sequence of the fibration associated to a continuous group action collapses at the $E_{2}$-stage, and their equivariant cohomology rings are free $H_{T}^{*}(\mathrm{pt})$-modules [35, Ch 3, Theorem 4.2]. To summarize, we have

$$
\begin{aligned}
& H_{T}^{*}\left(F \operatorname{lag}\left(\mathbb{C}^{n}\right)\right) \cong H_{T}^{*}(\mathrm{pt}) \otimes_{\mathbb{Q}} H^{*}\left(F \operatorname{Fag}\left(\mathbb{C}^{n}\right)\right) \quad \text { as } H_{T}^{*}(\mathrm{pt}) \text {-modules, } \\
& H_{S}^{*}(\operatorname{Hess}(\mathrm{N}, h)) \cong H_{S}^{*}(\mathrm{pt}) \otimes_{\mathbb{Q}} H^{*}(\operatorname{Hess}(\mathrm{N}, h)) \quad \text { as } H_{S}^{*}(\mathrm{pt}) \text {-modules, }
\end{aligned}
$$

and we also have injections

$$
\begin{aligned}
& \iota_{1}: H_{T}^{*}\left(\operatorname{Flag}\left(\mathbb{C}^{n}\right)\right) \hookrightarrow H_{T}^{*}\left(\operatorname{Flag}\left(\mathbb{C}^{n}\right)^{T}\right), \\
& \iota_{2}: H_{S}^{*}(\operatorname{Hess}(\mathrm{N}, h)) \hookrightarrow H_{S}^{*}\left(\operatorname{Hess}(\mathrm{N}, h)^{S}\right)
\end{aligned}
$$

where all the maps are induced from the inclusions.

Thus, in order to analyze $H_{S}^{*}(\operatorname{Hess}(\mathrm{N}, h))$, it suffices to understand their restrictions to the $S$-fixed point set. This will be a fundamental strategy employed throughout this paper. As a consequence, it is important to explicitly describe the relevant fixed point sets, to which we now turn.

We begin with the most familiar special case, namely $\operatorname{Flag}\left(\mathbb{C}^{n}\right)$; the general case will be analyzed in Section 2.2, For the standard $T$-action on the ambient variety $F \operatorname{lag}\left(\mathbb{C}^{n}\right)$, it is well-known that the $T$-fixed point set Flag $\left(\mathbb{C}^{n}\right)^{T}$ can be identified with the permutation group $\mathfrak{S}_{n}$ on $n$ letters. Indeed, we now fix once and for all an identification

$$
\mathfrak{S}_{n} \stackrel{\cong}{\rightarrow} \operatorname{Flag}\left(\mathbb{C}^{n}\right)^{T}
$$

which takes a permutation $w \in \mathfrak{S}_{n}$ to the flag specified by $V_{i}:=\operatorname{span}_{\mathbb{C}}\left\{e_{w(1)}, \ldots, e_{w(i)}\right\}$, where $\left\{e_{1}, \ldots, e_{n}\right\}$ denotes the standard basis of $\mathbb{C}^{n}$. (Alternatively, given the usual identification of $F$ lag $\left(\mathbb{C}^{n}\right)$ with $\operatorname{GL}(n, \mathbb{C}) / B$, we take $w$ to the coset represented by the standard permutation matrix associated to $w$ whose $(w(j), j)$-th entry is required to be 1 for each $j$ and otherwise entries are 0.) Restricting our attention to the subtorus $S \subset T$, it is straightforward to check that the $S$-fixed point set Flag $\left(\mathbb{C}^{n}\right)^{S}$ of the flag variety Flag $\left(\mathbb{C}^{n}\right)$ are also given by the above set $\operatorname{Flag}\left(\mathbb{C}^{n}\right)^{T}$, i.e.

$$
\operatorname{Flag}\left(\mathbb{C}^{n}\right)^{S}=\operatorname{Flag}\left(\mathbb{C}^{n}\right)^{T} .
$$

From here it also quickly follows that

$$
\operatorname{Hess}(\mathrm{N}, h)^{S}=\operatorname{Hess}(\mathrm{N}, h) \cap\left(\operatorname{Flag}\left(\mathbb{C}^{n}\right)\right)^{T} .
$$

Thus the set of $S$-fixed point set $\operatorname{Hess}(\mathrm{N}, h)^{S}$ is a subset of Flag $\left(\mathbb{C}^{n}\right)^{T}$, and through our fixed identification $\operatorname{Flag}\left(\mathbb{C}^{n}\right)^{T} \cong \mathfrak{S}_{n}$ from (2.15) we henceforth view $\operatorname{Hess}(\mathrm{N}, h)^{S}$ as a subset of $\mathfrak{S}_{n}$.

Based on the above discussions, we may consider the commutative diagram

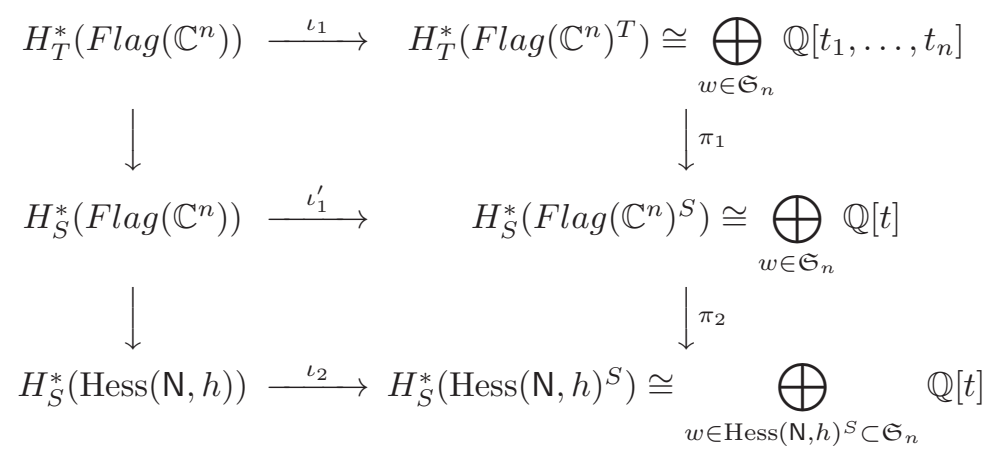

${ }^{2}$ For instance, it would certainly suffice if $X$ is locally contractible, compact, and Hausdorff. 
where all the maps are induced from the inclusion maps on underlying spaces. Note that all of $\iota_{1}, \iota_{1}^{\prime}$, and $\iota_{2}$ are injective as explained above since $\iota_{1}^{\prime}$ is a special case of $\iota_{2}$. The vertical map $\pi_{1}$ is given by $\pi_{1}=\bigoplus_{w \in \mathfrak{S}_{n}} \pi_{1}^{w}$, where $\pi_{1}^{w}: \mathbb{Q}\left[t_{1}, \ldots, t_{n}\right] \rightarrow \mathbb{Q}[t]$ is a ring homomorphism sending each $t_{i}$ to $i t$; this is because $\pi_{1}^{w}$ is induced by the projection map $\operatorname{Lie}(T)^{*} \rightarrow \operatorname{Lie}(S)^{*}$ and we use the identification (2.8). The vertical map $\pi_{2}$ is the obvious projection.

By slight abuse of notation, for $g \in H_{T}^{*}\left(F \operatorname{lag}\left(\mathbb{C}^{n}\right)\right)$ we denote its image $\iota_{1}(g)$ also by $g$. Also, for an element $g \in \bigoplus_{w \in \mathfrak{S}_{n}} \mathbb{Q}\left[t_{1}, \ldots, t_{n}\right]$ we will denote its $w$-th component by $g(w)$. Furthermore, we let $t_{i} \in \bigoplus_{w \in \mathfrak{S}_{n}} \mathbb{Q}\left[t_{1}, \ldots, t_{n}\right]$ denote the "constant polynomial" with value $t_{i}$ at each $w$, i.e. $t_{i}(w)=t_{i}$ for all $w \in \mathfrak{S}_{n}$. We apply the same convention for $H_{S}^{*}(\operatorname{Hess}(\mathrm{N}, h)) \stackrel{\iota_{2}}{\hookrightarrow} H_{S}^{*}\left(\operatorname{Hess}(\mathrm{N}, h)^{S}\right)$ and so we have the "constant" class

$$
t \in \bigoplus_{w \in \operatorname{Hess}(\mathrm{N}, h)^{S}} \mathbb{Q}[t] .
$$

This is the image of $t \in H^{2}(B S)$ (defined in (2.12) ) under the canonical homomorphism $H^{*}(B S) \rightarrow$ $H_{S}^{*}(\operatorname{Hess}(\mathrm{N}, h))$ composed with $\iota_{2}$. For the $T$-equivariant Chern class $\tau_{i}^{T} \in H_{T}^{2}\left(\operatorname{Flag}\left(\mathbb{C}^{n}\right)\right)$ introduced in (2.9), we have

$$
\tau_{i}^{T}(w)=t_{w(i)} \quad \text { for } w \in \mathfrak{S}_{n} .
$$

This can be seen by reading off the weight of the $T$-action on the fiber of the tautological line bundle $E_{i} / E_{i-1}$ over the permutation flag $V_{\bullet} \in F \operatorname{Flag}\left(\mathbb{C}^{n}\right)^{T}$ given by $V_{i}=\operatorname{span}_{\mathbb{C}}\left\{e_{w(1)}, \ldots, e_{w(i)}\right\}$.

2.2. The $S$-fixed point set of $\operatorname{Hess}(\mathrm{N}, h)$. The injectivity of $\iota_{2}$ in (2.14) shows that we can analyze $H_{S}^{*}(\operatorname{Hess}(\mathrm{N}, h))$ by viewing its elements as lists of polynomials with one coordinate for each (isolated) fixed point in $\operatorname{Hess}(\mathrm{N}, h)^{S}$. To successfully implement this strategy, we must understand the fixed point set Hess $(\mathbf{N}, h)^{S}$, viewed as a subset of $\mathfrak{S}_{n}$, in more detail. This is the goal of this section. In addition, we introduce some terminology associated to these fixed points, as well as a Hessenberg function $h_{w}$ associated to a permutation $w$ which will be useful for our later arguments. Indeed, it turns out that we can characterize $\operatorname{Hess}(\mathrm{N}, h)^{S}$ in terms of these functions $h_{w}$ (Proposition 2.9).

We will use the standard one-line notation $w=(w(1) w(2) \cdots w(n))$ for permutations in $\mathfrak{S}_{n}$. It will occasionally be convenient for us to think of permutations in $\mathfrak{S}_{n}$ as permutations on $\{0\} \cup[n]$, i.e. we use a convention

$$
w(0)=0 \quad \text { for all } w \in \mathfrak{S}_{n} .
$$

As a first step, we have the following.

Lemma 2.3. The $S$-fixed point set $\operatorname{Hess}(\mathrm{N}, h)^{S} \subset \mathfrak{S}_{n}$ of $\operatorname{Hess}(\mathrm{N}, h)$ is given by

$$
\operatorname{Hess}(\mathbf{N}, h)^{S}=\left\{w \in \mathfrak{S}_{n} \mid w^{-1}(w(j)-1) \leq h(j) \text { for all } j \in[n]\right\} .
$$

Proof. Since $\operatorname{Hess}(\mathrm{N}, h)^{S}=\operatorname{Hess}(\mathrm{N}, h) \cap\left(\operatorname{Flag}\left(\mathbb{C}^{n}\right)\right)^{T}$, it suffices to show that for any $w \in \mathfrak{S}_{n} \cong$ Flag $\left(\mathbb{C}^{n}\right)^{T}$, the condition $w^{-1}(w(j)-1) \leq h(j)(j=1,2, \ldots, n)$ is equivalent to the condition $w \in \operatorname{Hess}(\mathbf{N}, h)$. From (2.5) and (2.2) we see immediately that $w \in \operatorname{Hess}(\mathbf{N}, h)$ if and only if $w^{-1} \mathrm{~N} w \in H(h)$ where we regard $w$ as a permutation matrix, i.e. the matrix with $(w(j), j)$-th entry equal to 1 for each $j$ and all other entries equal to 0 . Since our $\mathbf{N}$ is the regular nilpotent matrix sending $e_{1} \mapsto 0$ and $e_{j} \mapsto e_{j-1}$ for $j>1$, we have that $\mathrm{N} w\left(e_{j}\right)=\mathbf{N}\left(e_{w(j)}\right)=0$ if $w(j)=1$ and $\mathbf{N} w\left(e_{j}\right)=e_{w(j)-1}$ if $w(j) \neq 1$. So $w^{-1} \mathbf{N} w\left(e_{j}\right)=0$ if $w(j)=1$ and $w^{-1} \mathrm{~N} w\left(e_{j}\right)=e_{w^{-1}(w(j)-1)}$ if $w(j) \neq 1$. Thus $w^{-1} \mathrm{~N} w \in H(h)$ precisely means that $w^{-1}(w(j)-1) \leq h(j)$ for all $j \in[n]$, where we follow the notational convention of (2.20).

In words, the condition in the above lemma can be stated as follows. Let $w \in \mathfrak{S}_{n}$ and let $w=$ $(w(1) w(2) \ldots w(n))$ be its one-line notation. Suppose that a consecutive pair of integers $k, k+1$ is inverted in the one-line notation of $w$, i.e. $k$ appears to the right of $k+1$, and suppose in this situation that $k+1$ appears in the $j$-th place (so $w(j)=k+1$ ) while $k$ appears in the $\ell$-th place (so $w(\ell)=k=w(j)-1$ ). Then the requirement of the condition is that $\ell \leq h(j)$. Informally, the Hessenberg function gives a restriction on "how far to the right" of $w(j)=k+1$ the value $w(j)-1=k$ is allowed to appear. Note that, for any $j$, if the pair $w(j)=k+1$ and $w(j)-1=k$ are not inverted in the one-line notation of $w$, i.e. $k$ appears 
to the left of $k+1$, then the condition is immediate, since by definition Hessenberg functions satisfy $h(j) \geq j$.

The inverted pairs $(k+1, k)$ play a special role in analyzing the $S$-fixed point set of $\operatorname{Hess}(\mathrm{N}, h)$. Motivated by this, we introduce some terminology.

Definition 2.4. Let $w \in \mathfrak{S}_{n}$ be a permutation and let $i, j \in[n]$. We say that $\mathcal{P}=(i, j)$ is an $\mathbf{N}$-inversion if $i<j$ and $w(i)=w(j)+1$. We refer to $i$ (respectively $j$ ) as the left (respectively right) position of the $\mathrm{N}$-inversion. Given an $\mathrm{N}$-inversion $\mathcal{P}=(i, j)$ we let $L P(\mathcal{P}):=i$ denote its left position and $R P(\mathcal{P}):=j$ its right position.

Given a permutation $w \in \mathfrak{S}_{n}$ we now define

$$
D_{w}:=\{\mathcal{P}=(i, j) \in[n] \times[n] \mid \mathcal{P} \text { is an N-inversion in } w\} .
$$

In the following it will be useful to focus on certain subsets of $D_{w}$. Let $j \in[n]$. We define

$$
D_{w}(j):=\left\{\mathcal{P} \in D_{w} \mid 1 \leq L P(\mathcal{P}) \leq j \text { and } j<R P(\mathcal{P}) \leq n\right\} .
$$

In words, the set $D_{w}(j)$ consists of the $\mathrm{N}$-inverted pairs whose left position is at or to the left of the $j$-th place, and whose right position is strictly to the right of the $j$-th place. The following is a quick consequence of the definition.

Lemma 2.5. Let $w \in \mathfrak{S}_{n}$ and $j \in[n]$. Then $D_{w}(j)=\emptyset$ if and only if $\{w(1), w(2), \ldots, w(j)\}=\{1,2, \ldots, j\}$.

Proof. If $\{w(1), w(2), \ldots, w(j)\}=\{1,2, \ldots, j\}$ then clearly $D_{w}(j)=\emptyset$ from the definition. Now suppose $D_{w}(j)=\emptyset$. Take an element $w(p) \in\{w(1), w(2), \ldots, w(j)\}(1 \leq p \leq j)$. Suppose $q$ is such that $w(p)-1=$ $w(q)$. Then from the assumption that $D_{w}(j)=\emptyset$ we must have $q \leq j$. That is, if $w(p) \neq 1$, then $w(p)-1(=w(q))$ is also contained in $\{w(1), \ldots, w(j)\}$. This means that $\{w(1), \ldots, w(j)\}$ is of the form $\left\{k \in[n] \mid k \leq k_{0}\right\}$ for some $k_{0} \in[n]$, but since the cardinality is $j$, it has to be $\{w(1), \ldots, w(j)\}=\{1, \ldots, j\}$ as desired.

Our next step is to define a map $w \mapsto h_{w}$ which associates to any permutation $w \in \mathfrak{S}_{n}$ a Hessenberg function $h_{w}$. The Hessenberg function $h_{w}$ is the minimal Hessenberg function $h$ such that $w \in \operatorname{Hess}(\mathrm{N}, h)$, in a sense to be made precise below. Specifically, given $w \in \mathfrak{S}_{n}$ we define

$$
h_{w}(j):= \begin{cases}j & \text { if } D_{w}(j)=\emptyset \\ \left.\max \left\{R P(\mathcal{P}) \mid \mathcal{P} \in D_{w}(j)\right)\right\} & \text { if } D_{w}(j) \neq \emptyset .\end{cases}
$$

We first prove that the function $h_{w}$ thus defined is in fact a Hessenberg function.

Lemma 2.6. Let $h_{w}$ be as above. Then $h_{w} \in H_{n}$.

Proof. We must show that $h_{w}(i) \geq i$ for all $i \in[n]$ and $h_{w}(i+1) \geq h_{w}(i)$ for all $i \in[n-1]$. First notice that if $D_{w}(j) \neq \emptyset$, then every element of $\left\{R P(\mathcal{P}) \mid \mathcal{P} \in D_{w}(j)\right\}$ is $>j$ by definition of $D_{w}(j)$. Thus the first claim follows from the definition of $h_{w}$. Next we check the second claim. Fix an $i$ in $[n-1]$. We take cases. First suppose $D_{w}(i)=\emptyset$. Then $h_{w}(i)=i$ by definition of $h_{w}$ and since we have already seen that $h_{w}(i+1) \geq i+1$, we obtain $h_{w}(i) \leq h_{w}(i+1)$ as desired. Next suppose $D_{w}(i) \neq \emptyset$ and $D_{w}(i+1)=\emptyset$. By Lemma 2.5 this means $\{w(1), \ldots, w(i+1)\}=\{1,2, \ldots, i+1\}$ but $\{w(1), \ldots, w(i)\} \neq\{1, \ldots, i\}$. It follows that $D_{w}(i)$ consists of a single $\mathrm{N}$-inverted pair $\mathcal{P}$, and that $R P(\mathcal{P})=i+1$. In particular $h_{w}(i)=i+1$. Hence $h_{w}(i+1) \geq i+1=h_{w}(i)$ and the claim holds in this case. Finally suppose both $D_{w}(i) \neq \emptyset$ and $D_{w}(i+1) \neq \emptyset$. If the $\mathrm{N}$-inverted pair achieving the maximum of the right position of $D_{w}(i)$ is also an element of $D_{w}(i+1)$, then clearly $h_{w}(i+1)=\max \left\{R P(\mathcal{P}) \mid \mathcal{P} \in D_{w}(i+1)\right\} \geq \max \left\{R P(\mathcal{P}) \mid \mathcal{P} \in D_{w}(i)\right\}=h_{w}(i)$ and the claim holds. Otherwise, the maximum of $\left\{R P(\mathcal{P}) \mid \mathcal{P} \in D_{w}(i)\right\}$ must be $i+1$, and $h_{w}(i)=i+1$. Since $h_{w}(i+1) \geq i+1$, the claim also holds in this case. We have checked all cases so this completes the proof.

The following reformulation of the definition of $h_{w}$ is sometimes useful. In the case when $D_{w}(j) \neq \emptyset$, it can be seen from the definitions that the value $h_{w}(j)$ may also be expressed as

$$
h_{w}(j)=\max \left\{w^{-1}(w(p)-1) \mid 1 \leq p \leq j\right\} \quad \text { if } D_{w}(j) \neq \emptyset .
$$


Note also that we have

$$
w^{-1}(w(j)-1) \leq h_{w}(j) \text { for all } j \in[n]
$$

by (2.22) together with the fact $w^{-1}(w(j)-1) \leq j$ if $D_{w}(j)=\emptyset$ by Lemma 2.5 (see also our convention $(2.201)$.

Before stating the next proposition we recall a natural partial ordering on Hessenberg functions.

Definition 2.7. Let $h^{\prime}, h \in H_{n}$. Then we say $h^{\prime} \subset h$ if $h^{\prime}(j) \leq h(j)$ for all $j \in[n]$.

The relation $h^{\prime} \subset h$ is evidently a partial order on $H_{n}$. Note that from the definition of $\operatorname{Hess}(\mathrm{N}, h)$ it is immediate that $h^{\prime} \subset h$ implies $\operatorname{Hess}\left(\mathrm{N}, h^{\prime}\right) \subset \operatorname{Hess}(\mathrm{N}, h)$ which explains our choice of notation.

Remark 2.8. Mbirika and Tymoczko [34] denote the above partial order with the symbol $\leq$ instead of the symbol $\subset$ which we use above. In later sections we additionally introduce a refinement of the above partial order to a total order $\preceq$.

With the terminology in place, we can give equivalent characterizations of the permutations $w \in \mathfrak{S}_{n}$ which lie in the $S$-fixed point set of $\operatorname{Hess}(\mathrm{N}, h)$.

Proposition 2.9. Let $w \in \mathfrak{S}_{n}$ and let $h \in H_{n}$. Then the following are equivalent:

(1) $w \in \operatorname{Hess}(\mathrm{N}, h)^{S}$,

(2) $w^{-1}(w(j)-1) \leq h(j)$ for all $j \in[n]$,

(3) $h_{w} \subset h$.

Proof. The equivalence of (1) and (2) is the content of Lemma 2.3 above. Also, it is easy to see that (3) implies (2) since we have (2.23) and by assumption we have $h_{w}(j) \leq h(j)$ for all $j \in[n]$. Hence it suffices to prove that (2) implies (3).

Suppose $w^{-1}(w(j)-1) \leq h(j)$ for all $j \in[n]$. We wish to prove that $h_{w}(j) \leq h(j)$ for all $j \in[n]$. We take cases. Suppose $D_{w}(j)=\emptyset$. Then $h_{w}(j)=j \leq h(j)$, where the inequality holds because $h \in H_{n}$. Hence the claim holds in this case. Now suppose $D_{w}(j) \neq \emptyset$. Then by (2.22) we have $h_{w}(j)=\max \left\{w^{-1}(w(p)-1) \mid 1 \leq\right.$ $p \leq j\}$ but the assumption shows $w^{-1}(w(p)-1) \leq h(p) \leq h(j)$ for all $p$ with $1 \leq p \leq j$. Hence $h_{w}(j) \leq h(j)$ also in this case.

For a fixed permutation $w \in \mathfrak{S}_{n}$, the above proposition implies that $h_{w}$ is the unique minimum with respect to the partial order $\subset$ in the set $\left\{h \in H_{n} \mid w \in \operatorname{Hess}(\mathrm{N}, h)^{S}\right\}$.

Finally, we record the following property of $h_{w}$ which we will use in Section 5 .

Lemma 2.10. Let $w \in \mathfrak{S}_{n}$ and $j \in[n-1]$. Suppose $D_{w}(j) \neq \emptyset\left(\right.$ i.e. $\left.h_{w}(j) \geq j+1\right)$. Then

$$
h_{w}(j)=w^{-1}(w(j)-1) \text { if and only if } h_{w}(j-1)<h_{w}(j) .
$$

Proof. First suppose $h_{w}(j-1)<h_{w}(j)$. We wish to show that $h_{w}(j)=w^{-1}(w(j)-1)$. We take cases. If $D_{w}(j-1) \neq \emptyset$, then by (2.22) we have

$$
h_{w}(j-1)=\max \left\{w^{-1}(w(p)-1) \mid 1 \leq p \leq j-1\right\}<\max \left\{w^{-1}(w(p)-1) \mid 1 \leq p \leq j\right\}=h_{w}(j)
$$

This implies that $h_{w}(j)=w^{-1}(w(j)-1)$. Next suppose that $D_{w}(j-1)=\emptyset$. Suppose in order to obtain a contradiction that $h_{w}(j) \neq w^{-1}(w(j)-1)$. By assumption, we have $D_{w}(j) \neq \emptyset$ so $h_{w}(j)=\max \left\{w^{-1}(w(p)-\right.$ 1) $\mid 1 \leq p \leq j\}$. If $h_{w}(j) \neq w^{-1}(w(j)-1)$ then the maximum must be achieved by a value $w^{-1}(w(p)-1)$ for $1 \leq p \leq j-1$, and since $h_{w}(j) \geq j$, this implies $D_{w}(j-1) \neq \emptyset$. This is a contradiction and we conclude $h_{w}(j)=w^{-1}(w(j)-1)$.

Now suppose $h_{w}(j)=w^{-1}(w(j)-1)$. We wish to show $h_{w}(j-1)<h_{w}(j)$. We again take cases. If $D_{w}(j-1)=\emptyset$, then by definition (2.21) of $h_{w}$, we have $h_{w}(j-1)=j-1<j \leq h_{w}(j)$, as desired. If $D_{w}(j-1) \neq \emptyset$, from (2.22) we have

$$
h_{w}(j-1)=\max \left\{w^{-1}(w(p)-1) \mid 1 \leq p \leq j-1\right\} .
$$

But from the assumption $D_{w}(j) \neq \emptyset$ and also from (2.22), we have

$$
h_{w}(j)=\max \left\{w^{-1}(w(p)-1) \mid 1 \leq p \leq j\right\}=w^{-1}(w(j)-1) .
$$

Hence, the maximum of the set is reached at $p=j$, implying that the values for $1 \leq p<j$ are strictly less than $w^{-1}(w(j)-1)$. Thus $h_{w}(j-1)<h_{w}(j)$ as desired. 


\section{Statement of Theorem 3.3. the equivariant version of Theorem A}

In this section we state the equivariant version of Theorem A. Consider the restriction homomorphism

$$
H_{T}^{*}\left(\operatorname{Flag}\left(\mathbb{C}^{n}\right)\right) \rightarrow H_{S}^{*}(\operatorname{Hess}(\mathrm{N}, h)) .
$$

For the tautological line bundle $E_{i} / E_{i-1}$ over $\operatorname{Flag}\left(\mathbb{C}^{n}\right)$ restricted to $\operatorname{Hess}(\mathrm{N}, h)$, let

$$
\bar{\tau}_{i}^{S} \in H_{S}^{2}(\operatorname{Hess}(\mathrm{N}, h)) \text { for } i \in[n]
$$

denote its $S$-equivariant first Chern class. That is, $\bar{\tau}_{i}^{S}$ is the image of $\tau_{i}^{T}$ (see (2.9)) under (3.1). We next analyze some algebraic relations satisfied by the $\bar{\tau}_{i}^{S}$. For this purpose, we now introduce some polynomials $f_{i, j}\left(x_{1}, \ldots, x_{n}, t\right) \in \mathbb{Q}\left[x_{1}, \ldots, x_{n}, t\right]$ for $n \geq i \geq j \geq 1$.

First we define

$$
p_{i}:=\sum_{k=1}^{i}\left(x_{k}-k t\right) \in \mathbb{Q}\left[x_{1}, \ldots, x_{n}, t\right] \quad \text { for } i \in[n] .
$$

For convenience we also set $p_{0}:=0$.

Definition 3.1. Let $(i, j)$ be a pair of natural numbers satisfying $n \geq i \geq j \geq 1$. We define polynomials $f_{i, j}$ inductively as follows. As the base case, when $i=j$, we define

$$
f_{j, j}:=p_{j} \quad \text { for } j \in[n] .
$$

Proceeding inductively, for $(i, j)$ with $n \geq i>j \geq 1$ we define

$$
f_{i, j}:=f_{i-1, j-1}+\left(x_{j}-x_{i}-t\right) f_{i-1, j}
$$

where we take the convention $f_{*, 0}:=0$ for any $*$.

Informally, we may visualize each $f_{i, j}$ as being associated to the lower-triangular $(i, j)$-th entry in an $n \times n$ matrix, as follows:

$$
\left(\begin{array}{ccccc}
f_{1,1} & 0 & \cdots & \cdots & 0 \\
f_{2,1} & f_{2,2} & 0 & \cdots & \\
f_{3,1} & f_{3,2} & f_{3,3} & \ddots & \\
\vdots & & & & \\
f_{n, 1} & f_{n, 2} & \cdots & & f_{n, n}
\end{array}\right) .
$$

Example 3.2. Suppose $n=4$. Then the $f_{i, j}$ have the following form.

$$
\begin{aligned}
& f_{i, i}=p_{i}(1 \leq i \leq 4) \\
& f_{2,1}=\left(x_{1}-x_{2}-t\right) p_{1} \\
& f_{3,2}=\left(x_{1}-x_{2}-t\right) p_{1}+\left(x_{2}-x_{3}-t\right) p_{2} \\
& f_{4,3}=\left(x_{1}-x_{2}-t\right) p_{1}+\left(x_{2}-x_{3}-t\right) p_{2}+\left(x_{3}-x_{4}-t\right) p_{3} \\
& f_{3,1}=\left(x_{1}-x_{3}-t\right)\left(x_{1}-x_{2}-t\right) p_{1} \\
& f_{4,2}=\left(x_{1}-x_{3}-t\right)\left(x_{1}-x_{2}-t\right) p_{1}+\left(x_{2}-x_{4}-t\right)\left\{\left(x_{1}-x_{2}-t\right) p_{1}+\left(x_{2}-x_{3}-t\right) p_{2}\right\} \\
& f_{4,1}=\left(x_{1}-x_{4}-t\right)\left(x_{1}-x_{3}-t\right)\left(x_{1}-x_{2}-t\right) p_{1}
\end{aligned}
$$

Now let $\mathbb{Q}\left[x_{1}, \ldots, x_{n}, t\right]$ denote the polynomial ring equipped with a grading defined by

$$
\operatorname{deg} x_{i}=2 \text { for all } i \in[n] \text { and } \operatorname{deg} t=2 .
$$

Note that $\mathbb{Q}\left[x_{1}, \ldots, x_{n}, t\right]$ is evidently a $\mathbb{Q}[t]$-algebra. We define a graded $\mathbb{Q}[t]$-algebra homomorphism $\tilde{\varphi}_{h}$ by

$$
\tilde{\varphi}_{h}: \mathbb{Q}\left[x_{1}, \ldots, x_{n}, t\right] \rightarrow H_{S}^{*}(\operatorname{Hess}(\mathrm{N}, h)) \quad ; \quad x_{i} \mapsto \bar{\tau}_{i}^{S}, t \mapsto t
$$

where $\bar{\tau}_{i}^{S}$ (defined in (3.2)) is the $S$-equivariant first Chern class of the tautological line bundle $E_{i} / E_{i-1}$ restricted to $\operatorname{Hess}(\mathrm{N}, h)$ and the class $t \in H_{S}^{*}(\operatorname{Hess}(\mathrm{N}, h))$ is the Chern class in (2.12). We are now ready to state the main technical result of this manuscript, the content of which is that the map $\tilde{\varphi}_{h}$ induces an isomorphism of graded $\mathbb{Q}[t]$-algebras between $H_{S}^{*}(\operatorname{Hess}(\mathrm{N}, h))$ and the quotient of $\mathbb{Q}\left[x_{1}, \ldots, x_{n}, t\right]$ by the ideal 
$I_{h}$ generated by a certain subset of the polynomials $f_{i, j}$ defined above. The proof of Theorem 3.3 occupies Sections 4 through 7.

Theorem 3.3. Let $n$ be a positive integer and $h:[n] \rightarrow[n]$ a Hessenberg function. Let Hess $(\mathrm{N}, h) \subset$ Flag $\left(\mathbb{C}^{n}\right)$ denote the corresponding regular nilpotent Hessenberg variety equipped with the action of the 1 dimensional subgroup $S$ described in Section 2.1. Then the restriction map

$$
H_{T}^{*}\left(\operatorname{Flag}\left(\mathbb{C}^{n}\right)\right) \rightarrow H_{S}^{*}(\operatorname{Hess}(\mathrm{N}, h))
$$

is surjective, and there is an isomorphism of graded $\mathbb{Q}[t]$-algebras

$$
H_{S}^{*}(\operatorname{Hess}(\mathrm{N}, h)) \cong \mathbb{Q}\left[x_{1}, \ldots, x_{n}, t\right] / I_{h}
$$

sending $x_{i}$ to $\bar{\tau}_{i}^{S}$ and t to $t$, where $\bar{\tau}_{i}^{S}$ (defined in (3.2)) is the $S$-equivariant first Chern class of the tautological line bundle restricted to $\operatorname{Hess}(\mathrm{N}, h)$ and we identify $H^{*}(B S) \cong \mathbb{Q}[t]$. Here the ideal $I_{h}$ is defined by

$$
I_{h}:=\left(f_{h(j), j} \mid 1 \leq j \leq n\right) .
$$

Using the association of the polynomials $f_{i, j}$ with the $(i, j)$-th entry of the matrix (3.5), the ideal $I_{h}$ can visually be described as being generated by the $f_{i, j}$ in the boxes at the bottom of each column in the the picture associated to the Hessenberg subspace $H(h)$ defined in (2.2) (see Figure 1). For instance, when $h=(3,3,4,5,6,6)$, the generators are $f_{3,1}, f_{3,2}, f_{4,3}, f_{5,4}, f_{6,5}, f_{6,6}$.

Remark 3.4. The above generalizes known results. Specifically, the special case when $h(j)=j+1$ for $j \in[n-1]$ has been well-studied and the corresponding variety $\operatorname{Hess}(\mathrm{N}, h)$ is called the Peterson variety Pet $_{n}$ (in Lie type A). Our result above generalizes a known presentation of $H_{S}^{*}\left(\right.$ Pet $\left._{n}\right)$ [15]. Indeed, for $1 \leq j \leq n-1$, we obtain from (3.4) and (3.3) that

$$
f_{j+1, j}=f_{j, j-1}+\left(-p_{j-1}+2 p_{j}-p_{j+1}-2 t\right) p_{j}
$$

and since $f_{n, n}=p_{n}$ we can inductively see that

$$
H_{S}^{*}\left(\text { Pet }_{n}\right) \cong \mathbb{Q}\left[p_{1}, \ldots, p_{n-1}, t\right] /\left(\left(-p_{j-1}+2 p_{j}-p_{j+1}-2 t\right) p_{j} \mid 1 \leq j \leq n-1\right) .
$$

where we take the convention $p_{0}=p_{n}=0$. This agrees with 15 .

Also, when $h(i)=n$ for $i \in[n]$, the associated variety is the full flag variety Flag $\left(\mathbb{C}^{n}\right)$. In this case, we will see later (in (6.8) and (6.9) ) an explicit relationship between the generators $\check{f}_{n, j}$ of our ideal $\check{I}_{h}=\check{I}_{(n, n, \ldots, n)}$ and the power sums $\mathrm{p}_{j}(x)=\mathrm{p}_{j}\left(x_{1}, \ldots, x_{n}\right):=\sum_{k=1}^{n} x_{k}^{j}$, thus relating our presentation with the usual Borel presentation of the cohomology of Flag $\left(\mathbb{C}^{n}\right)$ as in (2.11).

\section{Properties of the $f_{i, j}$}

In this section, in preparation for the proof of Theorem 3.3. we further analyze the polynomials $f_{i, j}$ defined in Definition 3.1. The results in this section, particularly Corollary 4.4 set the stage for the proof in Section 5 that the map $\tilde{\varphi}_{h}$ of (3.6) induces a well-defined map

$$
\varphi_{h}: \mathbb{Q}\left[x_{1}, \ldots, x_{n}, t\right] / I_{h} \rightarrow H_{S}^{*}(\operatorname{Hess}(\mathrm{N}, h)) .
$$

We begin with the following.

Lemma 4.1. The ideal $I_{h}$ defined in (3.7) contains $f_{i, j}$ for all $i \geq h(j)$. In particular, if $h \subset h^{\prime}$ in the sense of Definition 2.7, then $I_{h^{\prime}} \subset I_{h}$.

Proof. We prove that $I_{h}$ contains $f_{i, j}$ for all $i \geq h(j)$ by induction on $j$. When $j=1$, by the recursive relation (3.4) we have

$$
f_{i+1,1}=\left(x_{1}-x_{i+1}-t\right) f_{i, 1},
$$

and hence the assumption $f_{h(1), 1} \in I_{h}$ implies that $f_{i, 1} \in I_{h}$ for $i \geq h(1)$.

Now, assume that the claim holds for $j-1$, that is, $f_{i, j-1} \in I_{h}$ for all $i \geq h(j-1)$. We show that $f_{i, j} \in I_{h}$ for all $i \geq h(j)$. Since $f_{h(j), j} \in I_{h}$ by definition, again by induction on $i$, we may suppose $f_{i, j} \in I_{h}$ for some $i \geq h(j)$ and then we must prove that $f_{i+1, j} \in I_{h}$. By the recursive relation (3.4), we have

$$
f_{i+1, j}=f_{i, j-1}+\left(x_{j}-x_{i+1}-t\right) f_{i, j} .
$$


Since we have $i \geq h(j) \geq h(j-1)$, the inductive hypothesis implies that the RHS of this identity is contained in $I_{h}$, and hence we obtain $f_{i+1, j} \in I_{h}$ as desired.

The definition of $\tilde{\varphi}_{h}$ in (3.6) sends the variables $x_{i}$ to $\bar{\tau}_{i}^{S}$. In the next section we will prove that this map sends each $f_{h(j), j}$ to zero. In preparation for this, we investigate below some general properties of $f_{i, j}(w)$, i.e. the $w$-th component of $f_{i, j}$, at each fixed point $w \in F \operatorname{lag}\left(\mathbb{C}^{n}\right)^{S}=\mathfrak{S}_{n}$. More precisely, letting

$$
\tau_{i}^{S} \in H_{S}^{*}\left(\operatorname{Flag}\left(\mathbb{C}^{n}\right)\right)
$$

be the $S$-equivariant first Chern class of the tautological line bundle $E_{i} / E_{i-1}$ over $\operatorname{Flag}\left(\mathbb{C}^{n}\right)$ described in Section 2 we study the image of $f_{i, j}\left(\tau_{1}^{S}, \ldots, \tau_{n}^{S}, t\right)$ under the localization map

$$
\iota_{1}^{\prime}: H_{S}^{*}\left(\operatorname{Flag}\left(\mathbb{C}^{n}\right)\right) \rightarrow \bigoplus_{w \in \mathfrak{S}_{n}} \mathbb{Q}[t] .
$$

For the rest of this section, by slight abuse of notation we write

$$
f_{i, j}=f_{i, j}\left(\tau_{1}^{S}, \cdots, \tau_{n}^{S}, t\right)
$$

i.e. the elements of $H_{S}^{*}\left(F \operatorname{lag}\left(\mathbb{C}^{n}\right)\right)$ obtained by "evaluating at the $\tau_{i}^{S}$ ". Fix $w \in \mathfrak{S}_{n}$ and denote by $f_{i, j}(w) \in$ $\mathbb{Q}[t]$ the $w$-th component of the image of $f_{i, j}$ under the localization map $\iota_{1}^{\prime}$ above. Recall from Section 2 that the $w$-th component of the map $\pi_{1}=\bigoplus_{w \in \mathfrak{S}_{n}} \pi_{1}^{w}$ in the commutative diagram (2.17) is a ring homomorphism sending each $t_{i}$ to $i t$. Combined with (2.19), this implies that

$$
\tau_{i}^{S}(w)=w(i) t \quad \text { for } i \in[n] .
$$

It then follows from the definition of the $f_{i, j}$ (Definition 3.1) that

$$
\begin{aligned}
f_{j, j}(w) & =\sum_{k=1}^{j}(w(k)-k) t \quad(1 \leq j \leq n), \\
f_{i, j}(w) & =f_{i-1, j-1}(w)+(w(j)-w(i)-1) t \cdot f_{i-1, j}(w) \quad(n \geq i>j \geq 1) .
\end{aligned}
$$

The inductive nature of the $f_{i, j}$ allows us to conclude the following.

Lemma 4.2. Let $h \in H_{n}$. Let $f_{i, j}=f_{i, j}\left(\tau_{1}^{S}, \ldots, \tau_{n}^{S}, t\right)$ be defined as above. If $f_{h(j), j}(w)=0$ for all $j \in[n]$, then $f_{i, j}(w)=0$ for all $j \in[n]$ and $i \geq h(j)$.

Proof. Recall that $I_{h}$ is by definition the ideal of $\mathbb{Q}\left[x_{1}, \ldots, x_{n}, t\right]$ generated by the $f_{h(j), j}$ for $j \in[n]$. From Lemma 4.1 we know that if $i \geq h(j)$ then $f_{i, j} \in I_{h}$. By assumption, each $f_{h(j), j}$ lies in the kernel of the ring homomorphism

$$
\mathbb{Q}\left[x_{1}, \ldots, x_{n}, t\right] \rightarrow H_{S}^{*}\left(\operatorname{Flag}\left(\mathbb{C}^{n}\right)\right) \rightarrow \mathbb{Q}[t]
$$

where the first arrow sends $x_{i}$ to $\tau_{i}^{S}$ and the second map is the $w$-th coordinate of the localization map in (4.1). Thus the ideal $I_{h}$ also lies in the kernel as well, and hence also $f_{i, j}$ for $i \geq h(j)$.

To motivate the following discussion, it is useful to observe some properties of the $f_{i, j}$ for $j=1$. For simplicity, we use the notation

$$
u_{1}:=(w(1)-1) t
$$

Since $f_{*, 0}=0$ for any $*$, for the case $j=1$ the inductive description in (4.3) simplifies, and it is easy to see that for $i \geq 2$ we have

$$
f_{i, 1}(w)=u_{1} \prod_{k=2}^{i}\left(u_{1}-w(k) t\right)=\sum_{k=1}^{i}(-1)^{i-k} e_{i-k}(w(2), \ldots, w(i)) t^{i-k} u_{1}^{k}
$$

where $e_{\ell}$ denotes the $\ell$-th elementary symmetric polynomial in the given variables, and we take $e_{0}:=1$ by convention. Note that $f_{1,1}(w)=u_{1}$ by definition, so by the above convention on $e_{0}$, the equation (4.4) also holds for $i=1$.

The above computation turns out to be a special case of a general phenomenon, recorded in Lemma 4.3 In order to state and prove the result, we need to first introduce and study some properties of a new set of polynomials. 
Let $\mathbb{Q}\left[u_{1}, \ldots, u_{n}, t\right]$ be a graded polynomial ring of indeterminates $u_{1}, \ldots, u_{n}, t$ with $\operatorname{deg} u_{i}=2$ for $i \in[n]$ and $\operatorname{deg} t=2$. We inductively define a collection of polynomials $b_{k, j} \in \mathbb{Q}\left[u_{1}, \ldots, u_{n}, t\right]$ for $n \geq k \geq j \geq 1$ as follows. First define

$$
b_{j, j}:=\sum_{k=1}^{j}\left(u_{k}-(k-1) t\right) \quad \text { for all } j \in[n] .
$$

Then we define $b_{k, j}$ for $k \geq j$ by the equation

$$
b_{k+1, j}:=b_{k, j-1}+u_{j} b_{k, j}-\left(u_{j}+t\right) b_{k-1, j-1}
$$

where by convention we take $b_{*, 0}:=0$ for any $*$. Note that $b_{k, j}=b_{k, j}\left(u_{1}, \ldots, u_{j}, t\right)$ depends only on $u_{1}, \ldots, u_{j}$ and $t$, and $\operatorname{deg} b_{k, j}=2(k-j+1)=\operatorname{deg} f_{k, j}$ for $k \geq j$ since $\operatorname{deg} t=2$.

The above observation on $f_{i, 1}$ generalizes to the following property of $f_{i, j}$.

Lemma 4.3. Let $b_{k, j}$ be defined as above. Then for any pair $k, j \in[n]$ with $k \geq j$ the function $b_{k, j}$ is a symmetric polynomial in the variables $u_{1}, \ldots, u_{j}$. Moreover, we have the following equality in $\mathbb{Q}[t]$;

$$
f_{i, j}(w)=\sum_{k=j}^{i}(-1)^{i-k} e_{i-k}(w(j+1), \ldots, w(i)) t^{i-k} b_{k, j}((w(1)-1) t, \ldots,(w(j)-1) t, t) .
$$

We will use this property and the following Corollary in the next section to prove that $\tilde{\varphi}_{h}$ of (3.6) sends each $f_{h(j), j}$ to zero, but we postpone the highly technical proof to the Appendix.

Corollary 4.4. Let $m \in[n-1]$ and $w \in \mathfrak{S}_{n}$. If $w^{\prime}$ is the permutation obtained by interchanging $w(m)$ and $w(m+1)$, then we have $f_{i, j}\left(w^{\prime}\right)=f_{i, j}(w)$ for $i, j \in[n]$ with $i \geq j$ and $i \neq m, j \neq m$.

Proof. From (4.7) it follows that $f_{i, j}(w)$ depends only on $\{w(1), \ldots, w(i)\}$. Thus, if $i<m$ then since $f_{i, j}$ is independent of both $w(m)$ and $w(m+1)$, the claim follows trivially. If $m<j$ then $w(m), w(m+1) \in$ $\{w(1), \ldots, w(j)\}$, and since the $b_{k, j}$ are symmetric by Lemma 4.3 the claim follows. If $j<m<i$ then $w(m), w(m+1) \in\{w(j+1), \ldots, w(i)\}$, and since the $e_{i-k}$ are also symmetric, the result follows.

\section{First part of Proof of Theorem 3.3. Well-Definedness}

In order to prove that the homomorphism $\tilde{\varphi}_{h}$ defined in (3.6) induces a well-defined homomorphism

$$
\varphi_{h}: \mathbb{Q}\left[x_{1}, \ldots, x_{n}, t\right] / I_{h} \rightarrow H_{S}^{*}(\operatorname{Hess}(\mathrm{N}, h)) \quad ; \quad x_{i} \mapsto \bar{\tau}_{i}^{S}, t \mapsto t,
$$

it suffices to show that the polynomials $f_{h(j), j}$ generating the ideal $I_{h}$ lie in the kernel of the map $\tilde{\varphi}_{h}$ : $\mathbb{Q}\left[x_{1}, \ldots, x_{n}, t\right] \rightarrow H_{S}^{*}(\operatorname{Hess}(\mathrm{N}, h))$ given in (3.6). By the commutative diagram (2.17) and in particular by the injectivity of the bottom horizontal map $\iota_{2}$, it in turn suffices to show that $f_{h(j), j}(w)=0$ for any fixed point $w \in \operatorname{Hess}(\mathrm{N}, h)^{S}$. This is the content of Proposition 5.3 below, whose proof occupies the bulk of this section.

For the purposes of the argument below it is useful to introduce the following terminology. Consider the pairs $(i, j)$ for $i, j \in[n]$ in bijective correspondence with the entries in an $n \times n$ matrix. For a fixed integer $\ell \geq 0$, we refer to the pairs $\{(i, j) \mid i>j, i-j=\ell\}$ as the $\ell$-th lower diagonal. We say that the $\ell$-th lower diagonal is lower than the $k$-th lower diagonal if $\ell>k$. For example, the dots in Figure 2 indicate the $\ell$-th lower diagonals for $\ell=1$ and $\ell=2$.

Given a Hessenberg function $h$, we have already defined a corresponding Hessenberg subspace $H(h)$ (Definition 2.2). We can then ask which is the lowest lower diagonal which the Hessenberg subspace meets. More precisely, we say that a Hessenberg function meets the $\ell$-th lower diagonal if there exists some $j \in[n]$ such that $h(j)-j \geq \ell$. For example, for $h=(2,3,4,5,5)$ a Peterson Hessenberg function, the Hessenberg subspace meets the 0 -th and 1st lower diagonals, whereas for $h=(3,4,4,5,5)$, the Hessenberg subspace also meets the 2nd lower diagonal. The lowest lower diagonal which $h$ meets is evidently $\max _{j \in[n]}\{h(j)-j\}$. Finally, we shall say that $m$ is the last time that $h$ meets its lowest lower diagonal if $\ell=\max _{j \in[n]}\{h(j)-j\}$ and $m=\max _{j \in[n]}\{j \mid h(j)-j=\ell\}$. See Figure 2,

The following lemma proven by Drellich will be useful to prove Proposition 5.3 below. Recall from (2.1) that $H_{n}$ is the set of Hessenberg functions on $[n]$. 

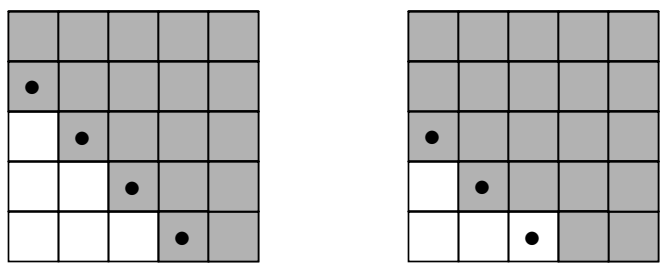

Figure 2. $h=(2,3,4,5,5)$ meets the 1st lower diagonal and $m=4$ (on the left), and $h=(3,4,4,5,5)$ meets the 2 nd lower diagonal and $m=2$ (on the right).

Lemma 5.1. (14, Theorem 4.5]) Let $h \in H_{n}$. Suppose $h(r)=r$ for some $r$ and let $h_{1}=(h(1), \ldots, h(r))$ and $h_{2}=(h(r+1)-r, \ldots, h(n)-r)$. Then $h_{1} \in H_{r}$ and $h_{2} \in H_{n-r}$. Moreover, for any $V_{\bullet} \in \operatorname{Hess}(\mathrm{N}, h)$ we have $V_{r}=\mathbb{C}^{r}=\mathbb{C} e_{1} \oplus \cdots \oplus \mathbb{C} e_{r}$ where $e_{1}, \ldots, e_{n}$ denote the standard basis of $\mathbb{C}^{n}$. In particular $\operatorname{Hess}(\mathrm{N}, h) \cong \operatorname{Hess}\left(\mathrm{N}_{1}, h_{1}\right) \times \operatorname{Hess}\left(\mathrm{N}_{2}, h_{2}\right)$ where $\mathrm{N}_{1}$ and $\mathrm{N}_{2}$ are the regular nilpotent matrices in Jordan canonical form of size $r$ and $n-r$, respectively.

The following is straightforward and will be used later.

Corollary 5.2. Let $h \in H_{n}$. Suppose $h(r)=r$ for some $r$ and let $h_{1}, h_{2}$ be as above. Let $S \subset T$ be the subtorus defined in (2.7). The $S$-action on $\operatorname{Hess}(\mathrm{N}, h)$ preserves each factor in the decomposition $\operatorname{Hess}(\mathrm{N}, h) \cong$ $\operatorname{Hess}\left(\mathrm{N}_{1}, h_{1}\right) \times \operatorname{Hess}\left(\mathrm{N}_{2}, h_{2}\right)$. In particular, $\operatorname{Hess}(\mathrm{N}, h)^{S} \cong \operatorname{Hess}\left(\mathrm{N}_{1}, h_{1}\right)^{S_{1}} \times \operatorname{Hess}\left(\mathrm{N}_{2}, h_{2}\right)^{S_{2}}$ where $S_{1}, S_{2}$ are the subgroups of $\mathrm{GL}(r, \mathbb{C})$ and $\mathrm{GL}(n-r, \mathbb{C})$ respectively defined in the same manner as in (2.7).

We are ready for the main assertion of this section.

Proposition 5.3. Let $h \in H_{n}$ and $j \in[n]$. If $w \in \operatorname{Hess}(\mathrm{N}, h)^{S}$, then $f_{h(j), j}(w)=0$.

Proof. We will first reduce the argument to the case when $n \geq 2$ and $h(j) \geq j+1$ for all $j \in[n-1]$. To see this, suppose that $n=1$. Then $\operatorname{Hess}(\mathrm{N}, h)=\operatorname{Flag}\left(\mathbb{C}^{1}\right)=\{\mathrm{id}\}$ where id $\in \mathfrak{S}_{1}$ is the identity permutation. Hence, in this case the claim is obvious by the recursive description (4.3) of $f_{j, j}$ (id). Now suppose that $n>1$ and that the claim holds for all $n^{\prime}<n$. Suppose also there exists $r, 1 \leq r<n$, such that $h(r)=r$ and without loss of generality let $r$ be the smallest such. From Corollary 5.2 we have that in (writing permutations in one-line notation)

(5.2) $\operatorname{Hess}(\mathrm{N}, h)^{S}=\left\{(u(1) \ldots u(r) v(1)+r \ldots v(n-r)+r) \in \mathfrak{S}_{n} \mid u \in \operatorname{Hess}\left(\mathrm{N}_{1}, h_{1}\right)^{S_{1}}, v \in \operatorname{Hess}\left(\mathrm{N}_{2}, h_{2}\right)^{S_{2}}\right\}$

where $S_{1} \subset \mathrm{GL}(r, \mathbb{C})$ and $S_{2} \subset \mathrm{GL}(n-r, \mathbb{C})$ are as in Corollary 5.2 By assumption on $r$ and the definition of Hessenberg functions we have that if $1 \leq j \leq r$ then $1 \leq h(j) \leq r$, and if $r+1 \leq j \leq n$ then $r+1 \leq h(j) \leq n$. Now let $w \in \operatorname{Hess}(\mathrm{N}, h)^{S}$. We wish to show that $f_{h(j), j}(w)=0$ for all $j \in[n]$. First consider the case when $1 \leq j \leq r$. By Lemma 4.3 we know $f_{h(j), j}(w)$ depends only on the values $\{w(1), \ldots, w(h(j))\}$. By assumption on $h$ and $j$ we know $h(j) \leq r$, so $f_{h(j), j}(w)$ depends only on $\{w(1), \ldots, w(r)\}$. Since $w \in \operatorname{Hess}(\mathrm{N}, h)^{S}$, from (5.2) we know $\{w(1), \ldots, w(r)\}=\{u(1), \ldots, u(r)\}$ for some $u \in \operatorname{Hess}\left(\mathrm{N}_{1}, h_{1}\right)^{S_{1}} \subseteq \mathfrak{S}_{r}$. Now the inductive hypothesis applied to $n^{\prime}=r<n$ implies $f_{h(j), j}(w)=f_{h_{1}(j), j}(u)=0$ as desired. Second, consider the case when $r+1 \leq j \leq n$. For this case, note first that since $h(j) \leq r$ for $j \leq r$, the above argument together with Lemma 4.2 implies that $f_{i, j}(w)=0$ for all $j \leq r$ and $i \geq r$. From the inductive definition of the $f_{i, j}$, it follows that for $j$ with $r+1 \leq j \leq n$, the value $f_{h(j), j}(w)$ agrees with the value of $f_{h_{2}(j-r), j-r}(v)$ where $v$ appears in (5.2). Since $n-r<n$, the inductive hypothesis again implies $f_{h(j), j}(w)=f_{h_{2}(j-r), j-r}(v)=0$ as desired.

An induction on $n$ and the argument above shows that it now suffices to prove the claim for the case when $n \geq 2$ and

$$
h(j) \geq j+1 \text { for all } j \in[n-1] .
$$

Fix such an $n$. The set of Hessenberg functions associated to $n$ which we must analyze is exactly

$$
H_{n}^{\prime}:=\left\{h \in H_{n} \mid h(j) \geq j+1 \text { for all } j \in[n-1]\right\} .
$$

The remainder of our argument is by induction using the total order on $H_{n}^{\prime}$, denoted by $\prec$, defined by

$$
h^{\prime} \prec h \Leftrightarrow \exists m \in[n-1] \text { such that for all } j \in[n] \text { with } j>m \text { we have } h^{\prime}(j)=h(j) \text {, and } h^{\prime}(m)<h(m) \text {. }
$$


The order $\prec$ is the usual reverse lexicographic order on $\mathbb{Z}_{\geq 0}^{n}$ if we view a Hessenberg function $h$ as a sequence $(h(1), h(2), \ldots, h(n))$ of positive integers. We also note that the above total order is a refinement of the partial order $h^{\prime} \subset h$ of Definition 2.7. Moreover, the unique minimal element in $H_{n}^{\prime}$ with respect to $\preceq$ is the Hessenberg function satisfying $h(j)=j+1$ for all $j \in[n-1]$. The base case of our induction therefore exactly corresponds to the Peterson variety, and as discussed in Remark 3.4 the results of [15 imply that the claim of the proposition holds in this case. Thus we may now assume that the claim is true for all $h^{\prime} \prec h$ and we must now prove the claim for $h$.

Suppose $w \in \operatorname{Hess}(\mathbf{N}, h)^{S}$. Then by Proposition 2.9 we know $h_{w} \subset h$, from which it follows that $h_{w} \preceq h$. If $h_{w} \neq h$, by the inductive hypothesis we may conclude that $f_{h_{w}(j), j}(w)=0$ for all $j$. From Lemma 4.2 and the definition of the partial order $h_{w} \subset h$ it then follows that $f_{h(j), j}(w)=0$ for all $j$, as desired. Thus it remains to check the claim for those $w$ with the property that $h=h_{w}$.

Since we may assume that $h=h_{w}$ is strictly larger than the Hessenberg function associated to the base case of the Peterson variety, there exists a $j$ such that $h(j) \geq j+2$. Note that such a $j$ must satisfy $j \leq n-2$ due to the definition of Hessenberg functions. Now let $m$ be the last time that $h=h_{w}$ meets its lowest lower diagonal, in the sense discussed above. By the above, such an $m$ must satisfy $h(m) \geq m+2$ and $m \leq n-2$. We also have that $h(m-1)<h(m)$, since otherwise $h$ meets a lower diagonal which is lower than that containing $(h(m), m)$, contradicting the definition of $m$. From Lemma 2.10 it then follows that $h(m)=h_{w}(m)=w^{-1}(w(m)-1)$, or in other words

$$
w(h(m))=w(m)-1 .
$$

Define a permutation $w^{\prime} \in \mathfrak{S}_{n}$ obtained from $w$ by interchanging the values in the $m$-th and $(m+1)$-st positions of the one-line notation of $w$, i.e. $w^{\prime}(m)=w(m+1), w^{\prime}(m+1)=w(m)$, and $w^{\prime}(j)=w(j)$ for all $j \neq m, m+1$. Let $h^{\prime}:=h_{w^{\prime}}$ denote the corresponding Hessenberg function.

We claim that

$$
h^{\prime}(j)=h(j) \text { for all } j \geq m+1 \text { and } h^{\prime}(m)<h(m) .
$$

To see this, first consider the case $j \geq m+1$. Recall that the definition of $h(j)=h_{w}(j)$ and $h^{\prime}(j)=h_{w^{\prime}}(j)$ is in terms of the sets $D_{w}(j), D_{w^{\prime}}(j)$ which are in turn constructed from the sets $D_{w}$ and $D_{w^{\prime}}$ by looking at $\mathrm{N}$-inverted pairs $\mathcal{P}$ with $L P(\mathcal{P}) \leq j$. Since $w$ and $w^{\prime}$ only differ in the $m$-th and $(m+1)$-st entries, if $j \geq m+1$ then from the definition it follows that $D_{w}(j)$ is obtained from $D_{w^{\prime}}(j)$ by replacing any $m$ which appears in a left position with an $m+1$, and hence $h(j)=h_{w}(j)=h_{w^{\prime}}(j)=h^{\prime}(j)$ as desired. Next, we wish to prove that $h^{\prime}(m)<h(m)$. To see this, first consider the case that $D_{w^{\prime}}(m)=\emptyset$. In this case, from the definition of $h^{\prime}=h_{w^{\prime}}$ we have $h^{\prime}(m)=m$, but since $h(m) \geq m+2$ as we observed above, we conclude $h^{\prime}(m)<h(m)$ as desired. Second, we consider the case $D_{w^{\prime}}(m) \neq \emptyset$. From (5.3) we know $(m, h(m)) \in D_{w}(m)$ and since $h(m)$ achieves the maximum of the set $\left\{R P(\mathcal{P}) \mid \mathcal{P} \in D_{w}(m)\right\}$ by definition (2.21), there are no N-inverted pairs $\mathcal{P} \in D_{w}(m)$ with $R P(\mathcal{P})>h(m)$. Now recall that we wish to show $h^{\prime}(m)<h(m)$ and we assume that $D_{w^{\prime}}(m) \neq \emptyset$. Since $w$ and $w^{\prime}$ swapped their $m$-th and $(m+1)$-st places, the pair $(m, h(m))$ is no longer an $\mathrm{N}$-inverted pair in $D_{w^{\prime}}(m)$. Let $p:=w^{-1}(w(m)+1)$ and $q:=w^{-1}(w(m+1)-1) \geq 0$ (see our convention (2.20) $)$. Then it follows that

$$
D_{w^{\prime}}(m) \subset\left(D_{w}(m) \backslash\{(m, h(m))\}\right) \cup\{(p, m+1)\} \cup\{(m, q)\} .
$$

We have

$$
q=w^{-1}(w(m+1)-1) \leq h_{w}(m+1)=h_{w}(m)=w^{-1}(w(m)-1)
$$

where the first inequality follows from (2.23) and the middle equality is because $m$ is the last time $h$ meets its lowest lower-diagonal. Since $w(m) \neq w(m+1)$ it cannot happen that $q=w^{-1}(w(m+1)-1)=w^{-1}(w(m)-1)$, so we conclude $q<h_{w}(m)=h(m)$. Since $(m, h(m)) \in D_{w}(m)$ and $D_{w^{\prime}}(m) \neq \emptyset$, by (2.21) we have $h_{w}(m)=\max R P\left(D_{w}(m)\right)$ and $h_{w^{\prime}}(m)=\max R P\left(D_{w^{\prime}}(m)\right)$. It then follows from (5.5) and $h(m) \geq m+2$ that $h^{\prime}(m)=h_{w^{\prime}}(m)<h_{w}(m)=h(m)$, as desired.

We have just seen that $h$ and $h^{\prime}$ agree in all coordinates to the right of $m$, and that $h^{\prime}(m)<h(m)$. Thus $h^{\prime} \prec h$, and by the inductive hypothesis, the claim of the proposition holds for $h^{\prime}$, so $f_{h^{\prime}(j), j}\left(w^{\prime}\right)=0$ for all $j$. Moreover, since $w^{\prime}$ is obtained from $w$ by interchanging the entries $w(m)$ and $w(m+1)$, from Corollary 4.4 we may conclude that $f_{h^{\prime}(j), j}(w)=0$ for all $j \geq m+1$. Since we have also seen above that $h(j)=h^{\prime}(j)$ for $j \geq m+1$, this implies $f_{h(j), j}(w)=0$ for all $j \geq m+1$. 
Next we compute for $j=m$. First, we have from the recursive relations (4.3) that

$$
\begin{aligned}
f_{h(m), m}(w) & =f_{h(m)-1, m-1}(w)+(w(m)-w(h(m))-1) t \cdot f_{h(m)-1, m}(w) \\
& =f_{h(m)-1, m-1}(w) \\
& =f_{h(m)-1, m-1}\left(w^{\prime}\right)
\end{aligned}
$$

where the second equality holds because we have shown that $w(m)-1=w(h(m))$ in (5.3) and the third equality follows by Corollary 4.4 since $h(m) \geq m+2$ so $h(m)-1 \neq m$. Recall that $h_{w^{\prime}}=h^{\prime} \prec h$, so by the inductive hypothesis we have that $f_{h^{\prime}(j), j}\left(w^{\prime}\right)=0$ for all $j$. But then by Lemma 4.2 we know that $f_{i, j}\left(w^{\prime}\right)=0$ for any $j \in[n]$ and $i \geq h^{\prime}(j)$. In particular, since $h^{\prime}(m-1) \leq h^{\prime}(m)$ by definition of Hessenberg functions and since $h^{\prime}(m) \leq h(m)-1$ as shown in (5.4), we have $h^{\prime}(m-1) \leq h(m)-1$ and hence $f_{h(m)-1, m-1}\left(w^{\prime}\right)=0$. From (5.6) this implies $f_{h(m), m}(w)=0$, as desired.

It remains to check that $f_{h(j), j}(w)=0$ for $j \leq m-1$. We will again argue by comparing the computations for $h^{\prime}$ with those for $h$. Note that in general it may not be the case that $h^{\prime} \subset h$. Recalling that $h^{\prime}=h_{w^{\prime}}$ is defined in terms of the permutation $w^{\prime}$ which differs from $w$ only in the $m$-th and $(m+1)$-st spots, it is useful to define

$$
r:=\min \{j \mid m \leq h(j)\}, \quad s:=\min \{j \mid m+1 \leq h(j)\} .
$$

We also define $r_{0}$ (respectively $s_{0}$ ) as the position of $w(m)+1$ (respectively $\left.w(m+1)+1\right)$ :

$$
w\left(r_{0}\right)-1=w(m)=w^{\prime}(m+1), \quad w\left(s_{0}\right)-1=w(m+1)=w^{\prime}(m) .
$$

It is clear from the definitions that $r_{0} \neq m$ and $s_{0} \neq m+1$. If $r_{0}<m$, then $\left(r_{0}, m\right)$ is an $\mathrm{N}$-inverted pair in $D_{w}\left(r_{0}\right)$, so $m \leq h_{w}\left(r_{0}\right)=h\left(r_{0}\right)$ by the definition (2.21) of $h_{w}$, and if $r_{0}>m$ then since $h\left(r_{0}\right)=h_{w}\left(r_{0}\right) \geq r_{0}$, we also have $m \leq h\left(r_{0}\right)$. Thus, from the definition (5.7) of $r$ we see that $r \leq r_{0}$. Similarly $s \leq s_{0}$. Moreover, since $h(m) \geq m+2$ and $m \leq n-2$ by the definition of $m$, we also have $r \leq s \leq m \leq n-2$. In summary, we have

$$
r \leq r_{0}, \quad s \leq s_{0}, \quad r \leq s \leq m \leq n-2 .
$$

Note also that from the definition of $r$ it follows that $h(r-1)<h(r)$. Furthermore, from (5.8) we know $r<n$ and hence from the original assumption on the Hessenberg function $h$ we know $h(r) \geq r+1$. Thus we may apply Lemma 2.10 to conclude that

$$
h(r)=w^{-1}(w(r)-1) \text { and hence } w(h(r))=w(r)-1 .
$$

That is, we have $(r, h(r)) \in D_{w}$.

The next observation will be useful in what follows. By assumption on the Hessenberg function $h$ we have $h(j) \geq j+1$ for all $j \leq n-1$ and hence from the definition (2.21) of $h=h_{w}$ we see that $D_{w}(j) \neq \emptyset$ for all $j \leq n-1$. Since $w^{\prime}$ and $w$ only differ in the $m$-th and $(m+1)$-st spots we also have $D_{w^{\prime}}(j) \neq \emptyset$ for $j \leq m-1$. Hence, the description (2.22) for $h_{w}$ and $h_{w^{\prime}}$ shows that for $j \leq m-1$ we can express $h(j)$ and $h^{\prime}(j)$ by

$$
\begin{aligned}
& h(j)=h_{w}(j)=\max \left\{w^{-1}(w(p)-1) \mid 1 \leq p \leq j\right\}, \\
& h^{\prime}(j)=h_{w^{\prime}}(j)=\max \left\{w^{\prime-1}\left(w^{\prime}(p)-1\right) \mid 1 \leq p \leq j\right\} .
\end{aligned}
$$

Recall that we wish to show $f_{h(j), j}(w)=0$ for $j \leq m-1$. We will argue on a case-by-case basis according to the value of $h(r)$, where $r$ is the value defined in (5.7).

Case 1. Suppose $h(r) \geq m+2$. Then from the definitions of $r$ and $s$ in (5.7) it immediately follows that $r=s$. We already know that $r \leq r_{0}$ from (5.8), but in this case from (5.9) we in fact have

$$
w^{-1}(w(r)-1)=h(r) \geq m+2>m=w^{-1}\left(w\left(r_{0}\right)-1\right)
$$

so $r \neq r_{0}$, from which it follows $r<r_{0}$. It similarly follows that $s=r<s_{0}$. From this we claim that $h(j)=h^{\prime}(j)$ for $j \leq m-1$. Indeed, recall from (5.10) that $h(j)$ (respectively $h^{\prime}(j)$ ) can be described as the maximum of the right positions $R P(\mathcal{P})$ of $\mathrm{N}$-inverted pairs $\mathcal{P}$ whose left positions go from 1 up to $j$. Our assumption that $h(r) \geq m+2$ implies that the one-line notation of $w$ is of the form

$$
(\ldots, w(r), \ldots, w(m), w(m+1), \ldots, w(h(r))=w(r)-1, \ldots)
$$

where the position $h(r)$ of $w(r)-1$ is, by assumption, to the right of both $w(m)$ and $w(m+1)$. We have just argued that $r<r_{0}$ and $r<s_{0}$, which is to say that if $w(m)$ and $w(m)+1$ (respectively $w(m+1)$ 
and $w(m+1)+1)$ appear in inverted order in $w$, then the larger value $w(m)+1=w\left(r_{0}\right)$ (respectively $\left.w(m+1)+1=w\left(s_{0}\right)\right)$ must appear to the right of $w(r)$. (If they do not appear in inverted order, then they cannot be an inverted pair and hence never contribute to the computation of $h=h_{w}$.) But since $h(j)$ is computed by looking for the maximum of the $R P(\mathcal{P})$ for such $\mathcal{P}$ whose left position is up to $j$, and since the $\mathrm{N}$-inverted pair $(r, h(r))$ occurs before $r_{0}$ and $s_{0}$ (i.e. $r<r_{0}$ and $\left.r<s_{0}\right)$ but has a larger $R P(\mathcal{P})$ (i.e. $w(h(r))=w(r)-1$ occurs to the right of $w(m)$ and $w(m+1)$ ), this implies that the inverted pairs (if any) with right positions $m$ and $m+1$ never achieve the maximum in the computation of $h(j)$. Since $w^{\prime}$ differs from $w$ only by interchanging the $w(m)$ and $w(m+1)$, this assertion remains true for $w^{\prime}$. Hence the computation for $h(j)$ and $h^{\prime}(j)$ remains unchanged, and we conclude

$$
h(j)=h^{\prime}(j) \text { for } j \leq m-1 .
$$

Now from the inductive hypothesis we know $f_{h^{\prime}(j), j}\left(w^{\prime}\right)=0$ for all $j \in[n]$. Since we just saw $h(j)=h^{\prime}(j)$ for $j \leq m-1$ in this case, we then obtain that $f_{h(j), j}\left(w^{\prime}\right)=0$ for $j \leq m-1$. Finally, observe that $h(j)<m$ for any $j<r$ by definition of $r$, and for $j \geq r$ the assumption that $h(r) \geq m+2$ implies that $h(j) \neq m$. Hence we may apply Corollary 4.4 and conclude that $f_{h(j), j}(w)=0$ for $j \leq m-1$, as desired.

Case 2. Next we consider the case $h(r)=m+1$. We immediately see that $r=s$ in this case as well. Recall from (5.9) that $h(r)=w^{-1}(w(r)-1)$. Since $h(r)=m+1$ by assumption we have $w(m+1)=w(r)-1$. Recall that the definition of $m$ guarantees that $h(m) \geq m+2$, so $r<m$. Based on this discussion we conclude that the one-line notation for $w$ looks like

$$
(\ldots, w(r)=w(m+1)+1, \ldots, w(m), w(m+1), \ldots)
$$

so we can see that $r=s=s_{0}$ in this case. Also, arguing as in the case above, we know that $r<r_{0}$. We claim that

$$
h^{\prime}(j) \leq h(j) \text { for all } j \leq m-1 \text {. }
$$

We take cases. Recall that $r_{0} \neq m$ since $r_{0}$ is defined by $w\left(r_{0}\right)=w(m)+1$. First suppose $r_{0} \geq m+1$, i.e. the value $w(m)+1$ occurs to the right of $w(m)$ in the one-line notation for $w$. In this case, the integers $w(m)$ and $w(m)+1$ are not inverted in $w$ and hence never contributes to the computation of any $h(j)$. Hence we may conclude (5.11) in this case. Next consider the case $r_{0} \leq m-1$, so the one-line notation for $w$ looks like

$$
\left(\ldots, w(r)=w(m+1)+1, \ldots, w\left(r_{0}\right)=w(m)+1, \ldots, w(m), w(m+1), \ldots\right)
$$

and the one-line notation for $w^{\prime}$ then looks like

$$
\left(\ldots, w^{\prime}(r)=w(m+1)+1, \ldots, w^{\prime}\left(r_{0}\right)=w(m)+1, \ldots, w(m+1), w(m), \ldots\right) .
$$

In what follows we prove (5.11) by looking at the one-line notations. Recall that the only difference between $w$ and $w^{\prime}$ is that $w(m)$ and $w(m+1)$ have been interchanged, and that the computation of $h(j)$ involves looking at $\mathrm{N}$-inverted pairs in $D_{w}$ with left position up to $j$ (and similarly for $h^{\prime}(j)$ ). For the cases $1 \leq j<r$ or $r_{0} \leq j \leq m-1$ (i.e. the cases in which $j$ is outside of the range between $r$ and $r_{0}-1$ ), we see from this observation together with the above one-line notations that $h^{\prime}(j)=h(j)$. For the case $r \leq j<r_{0}$, we have $h^{\prime}(j) \leq h(j)$ by the same reasoning. Thus we conclude (5.11), as desired.

Now from the inductive hypothesis we know that $f_{h^{\prime}(j), j}\left(w^{\prime}\right)=0$ for all $j$, so from Lemma 4.2 and (5.11) we may conclude that $f_{h(j), j}\left(w^{\prime}\right)=0$ for all $j \leq m-1$. From the assumption that $h(r)=m+1$ it follows as in the argument for Case 1 that there does not exist any $j$ with $h(j)=m$, and since $j \leq m-1$ we have $j \neq m$. Hence we may apply Corollary 4.4 to conclude that $f_{h(j), j}(w)=0$ for $j \leq m-1$, as desired. This completes the argument for Case 2.

Case 3. It remains to consider the case when $h(r)=m$. This means that $m$ is actually achieved as a value of $h$, so by the definition of $s$ it follows that $r \neq s, r<s$, and $h(s) \geq m+1$. From (5.9) we also know $w(r)-1=w(h(r))$, and since $h(r)=m$ we have $w(r)-1=w(m)$. Hence $r=r_{0}$. The one-line notation for $w$ therefore looks like

$$
\left(\ldots, w(r)=w\left(r_{0}\right)=w(m)+1, \ldots, w(s), \ldots, w(m), w(m+1), \ldots\right)
$$

and the one-line notation for $w^{\prime}$ looks like

$$
\left(\ldots, w(r)=w\left(r_{0}\right)=w(m)+1, \ldots, w(s), \ldots, w(m+1), w(m), \ldots\right)
$$


where the only difference is the interchanging of $w(m)$ and $w(m+1)$.

We now concretely analyze the difference between $h$ and $h^{\prime}$. Recalling $s \leq s_{0}$ from (5.8) where we recall that $s_{0}$ is the position of $w(m+1)+1$ in the one-line notation for $w$, it follows from arguments similar to those in the previous cases that

$$
\begin{aligned}
& h^{\prime}(j)=h(j)<m \quad \text { for } 1 \leq j \leq r-1, \\
& h^{\prime}(j)=m+1=h(j)+1 \quad \text { for } r \leq j \leq s-1, \text { and } \\
& \left.h^{\prime}(j)=h(j)>m \quad \text { for } s \leq j \leq m-1 \text { (note that if } s=m \text { then there are no such } j\right) .
\end{aligned}
$$

Now, consider $j$ with $1 \leq j \leq r-1$ or $s \leq j \leq m-1$. By (5.12) we have in these cases that $h^{\prime}(j)=h(j)$. Hence by the inductive hypothesis $f_{h(j), j}\left(w^{\prime}\right)=f_{h^{\prime}(j), j}\left(w^{\prime}\right)=0$. Moreover, since $j \neq m$ and $h(j) \neq m$ by (5.12), we may again apply Corollary 4.4 to conclude that $f_{h(j), j}(w)=0$, as desired.

It remains to consider the case of $j$ with $r \leq j \leq s-1$. For such $j$ we have $h(j)=m$, so we wish to prove that $f_{m, j}(w)=0$ for $r \leq j \leq s-1$. We argue by induction, with the base case being $j=r$. For what follows we introduce the temporary notation

$$
A_{j}:=f_{m-1, j}(w)=f_{m-1, j}\left(w^{\prime}\right) \quad(j \leq m-1) .
$$

where the second equality holds because $j \neq m$ by assumption and by Corollary 4.4. Also, from (5.12) we have that $h^{\prime}(r-1)=h(r-1) \leq m-1$, so from our inductive hypothesis on $h^{\prime}$ and Lemma 4.2 we may conclude $A_{r-1}=f_{m-1, r-1}\left(w^{\prime}\right)=0$. Since $m>r$, using the recursive equation (4.3) of the $f_{i, j}(w)$ we may now compute

$$
f_{m, r}(w)=f_{m-1, r-1}(w)+(w(r)-w(m)-1) f_{m-1, r}(w) t=A_{r-1}+(w(r)-w(m)-1) A_{r} t=0
$$

where we have used the fact that $r=r_{0}$ and thus $w(r)=w(m)+1$. This proves the claim for the base case $j=r$.

Now suppose by induction that $f_{m, k}(w)=0$ for some $k$ with $r \leq k \leq s-2$, and we wish to prove the statement for $k+1$. We know from (5.12) that $h^{\prime}(k+1)=m+1$, so from our inductive hypothesis on $h^{\prime}$ we have $f_{m+1, k+1}\left(w^{\prime}\right)=0$. Since $m>k+1$, using (repeatedly) the recursive equation (4.3) of the $f_{i, j}(w)$ and (5.13) we have

$$
\begin{aligned}
0= & f_{m+1, k+1}\left(w^{\prime}\right) \\
= & f_{m, k}\left(w^{\prime}\right)+\left(w^{\prime}(k+1)-w^{\prime}(m+1)-1\right) f_{m, k+1}\left(w^{\prime}\right) t \\
= & A_{k-1}+\left(w^{\prime}(k)-w^{\prime}(m)-1\right) A_{k} t \\
& \quad+\left(w^{\prime}(k+1)-w^{\prime}(m+1)-1\right)\left(A_{k}+\left(w^{\prime}(k+1)-w^{\prime}(m)-1\right) A_{k+1} t\right) t \\
= & A_{k-1}+(w(k)-w(m+1)+w(k+1)-w(m)-2) A_{k} t \\
& \quad+(w(k+1)-w(m)-1)(w(k+1)-w(m+1)-1) A_{k+1} t^{2}
\end{aligned}
$$

where the last equality also uses the definition of $w^{\prime}$ in terms of $w$. By our assumption on $k$ we have

$$
f_{m, k}(w)=A_{k-1}+(w(k)-w(m)-1) A_{k} t=0,
$$

and hence we can further simplify the last expression in (5.14) as

$$
0=(w(k+1)-w(m+1)-1)\left(A_{k}+(w(k+1)-w(m)-1) A_{k+1} t\right) t .
$$

Now remember that by assumption $k+1 \leq s-1$ and also $s \leq s_{0}$ from (5.8), which means $w(k+1) \neq$ $w\left(s_{0}\right)=w(m+1)+1$. Thus from (5.15) we finally obtain

$$
f_{m, k+1}(w)=A_{k}+(w(k+1)-w(m)-1) A_{k+1} t=0,
$$

as desired. This proves the result that $f_{h(j), j}(w)=0$ for all $r \leq j \leq s-1$, so we have checked all cases and the result is proved.

We now prove that the ring homomorphism (5.1) is well-defined. Recall that $I_{h}$ is the ideal of $\mathbb{Q}\left[x_{1}, \ldots, x_{n}, t\right]$ generated by $f_{h(j), j}$ for $j=1, \ldots, n$. 
Corollary 5.4. The graded $\mathbb{Q}[t]$-algebra homomorphism

$$
\varphi_{h}: \mathbb{Q}\left[x_{1}, \ldots, x_{n}, t\right] / I_{h} \rightarrow H_{S}^{*}(\operatorname{Hess}(\mathrm{N}, h))
$$

which sends each $x_{i}$ to the first Chern class $\bar{\tau}_{i}^{S}$ and $t$ to $t$ is well-defined, where we identify $H^{*}(B S) \cong \mathbb{Q}[t]$.

Proof. For $w \in \operatorname{Hess}(\mathrm{N}, h)^{S}$, the $w$-th component of the image of $f_{h(j), j}\left(\bar{\tau}_{1}^{S}, \ldots, \bar{\tau}_{n}^{S}, t\right)$ under the localization map $\iota_{2}: H_{S}^{*}(\operatorname{Hess}(\mathrm{N}, h)) \rightarrow \bigoplus_{w \in \operatorname{Hess}(\mathrm{N}, h)^{S}} \mathbb{Q}[t]$ is precisely the $f_{h(j), j}(w)$ considered in Proposition 5.3 Thus, Proposition 5.3 together with the injectivity of $\iota_{2}$ implies that

$$
f_{h(j), j}\left(\bar{\tau}_{1}^{S}, \ldots, \bar{\tau}_{n}^{S}, t\right)=0 \in H_{S}^{*}(\operatorname{Hess}(\mathrm{N}, h))
$$

for all $j \in[n]$. Hence, the ring homomorphism $\tilde{\varphi}_{h}$ defined in (3.6) factors through the quotient by $I_{h}$, inducing the map $\varphi_{h}$ as desired.

\section{HilberT SERIES}

The main result of this section, Proposition 6.12, takes a further step in the proof of Theorem 3.3 by proving that the two rings are additively isomorphic as graded $\mathbb{Q}$-vector spaces, i.e. that their Hilbert series (to be defined below) are equal. This will be useful in our arguments in Section 7 because, if a map between two graded vector spaces is injective and we know the dimensions of the graded pieces are equal, then the map must be an isomorphism.

We outline the content of this section. We first record some preliminary definitions and recall some properties of regular sequences. To prove Proposition 6.12 it will turn out to be useful to first compute the Hilbert series for the ordinary cohomology. As a first step, by using results of Mbirika-Tymoczko [34 and a small trick involving the Hessenberg space's negative roots, we rewrite the Hilbert series of the ordinary cohomology $H^{*}(\operatorname{Hess}(\mathrm{N}, h))$ in terms of $h$. Next, we show in Lemma 6.5 that the polynomials $\check{f}_{i, j}$ defined in (1.2) can be obtained from the $f_{i, j}$ by setting the variable $t$ equal to 0 , and then prove that the homogeneous polynomials $\check{f}_{h(1), 1}, \ldots, \check{f}_{h(n), n}$ described in (6.3) form a regular sequence in $\mathbb{Q}\left[x_{1}, \ldots, x_{n}\right]$. Since the degrees of the $\check{f}_{h(i), i}$ are known, this allows us to conclude that the Hilbert series of Hess $(\mathrm{N}, h)$ and $\mathbb{Q}\left[x_{1}, \ldots, x_{n}\right] / \check{I}_{h}$ are equal. Here recall that $\check{I}_{h}$, defined in (1.4), is the ideal

$$
\check{I}_{h}=\left(\check{f}_{h(1), 1}, \ldots, \check{f}_{h(n), n}\right)
$$

generated by the $f_{h(j), j}$ for $j \in[n]$. Now some straightforward arguments, involving on the one hand some elementary considerations using module bases and the $S$-equivariant formality of $\operatorname{Hess}(\mathrm{N}, h)$ on the other, yield the fact that the Hilbert series of the $S$-equivariant cohomology $H_{S}^{*}(\operatorname{Hess}(\mathrm{N}, h))$ and $\mathbb{Q}\left[x_{1}, \ldots, x_{n}, t\right] / I_{h}$ are equal.

As before, we equip the polynomial rings $\mathbb{Q}\left[x_{1}, \ldots, x_{n}\right]$ and $\mathbb{Q}\left[x_{1}, \ldots, x_{n}, t\right]$ with the gradings defined by

$$
\operatorname{deg} x_{i}=2 \text { for all } i \in[n] \quad \text { and } \quad \operatorname{deg} t=2 .
$$

We begin by recalling the definition of Hilbert series. Let $R=\bigoplus_{i=0}^{\infty} R_{i}$ be a graded $\mathbb{Q}$-vector space where each $R_{i}$ is finite-dimensional. Then we define its Hilbert series to be

$$
F(R, s):=\sum_{i=0}^{\infty}\left(\operatorname{dim}_{\mathbb{Q}} R_{i}\right) s^{i} \in \mathbb{Z}[[s]]
$$

where $s$ is a formal parameter. We also take a moment to recall the definition and some properties of regular sequences, which we use extensively for the remainder of this section.

Definition 6.1. ([32, Section 16]) For a ring $S$, a sequence $\theta_{1}, \ldots, \theta_{r} \in S$ is called a regular sequence if:

(i) $\theta_{i}$ is non-zero, and not a zero-divisor, in $S /\left(\theta_{1}, \ldots, \theta_{i-1}\right)$ for $i=1, \ldots, r$,

(ii) $S /\left(\theta_{1}, \ldots, \theta_{r}\right) \neq 0$.

Remark 6.2. There are other useful characterizations of regular sequences which we shall employ in our arguments below. 
(1) If $S$ is a graded $\mathbb{Q}$-algebra and $\theta_{1}, \ldots, \theta_{r}$ are positive-degree homogeneous elements, then it is wellknown that $\left\{\theta_{1}, \ldots, \theta_{r}\right\}$ is a regular sequence if and only if $\left\{\theta_{1}, \ldots, \theta_{r}\right\}$ is algebraically independent over $\mathbb{Q}$ and $S$ is a free $\mathbb{Q}\left[\theta_{1}, \ldots, \theta_{r}\right]$-module (e.g. [45. Chapter 1 , Section 5.6]).

(2) Let the polynomial ring $S=\mathbb{Q}\left[x_{1}, \ldots, x_{n}\right]$ be graded with $\operatorname{deg} x_{i}=2$ for $1 \leq i \leq n$. It is also well-known (see for instance [45, p.35]) that a sequence $\theta_{1}, \ldots, \theta_{r} \in \mathbb{Q}\left[x_{1}, \ldots, x_{n}\right]$ of positive-degree homogeneous polynomials is a regular sequence if and only if

$$
F\left(\mathbb{Q}\left[x_{1}, \ldots, x_{n}\right] /\left(\theta_{1}, \ldots, \theta_{r}\right), s\right)=\frac{1}{\left(1-s^{2}\right)^{n}} \prod_{k=1}^{r}\left(1-s^{\operatorname{deg} \theta_{k}}\right) .
$$

(3) Finally, continuing in the special setting of the previous item, if $r=n$ in addition, it is known that a sequence of positive-degree homogeneous elements $\theta_{1}, \ldots, \theta_{n}$ in $\mathbb{Q}\left[x_{1}, \ldots, x_{n}\right]$ is a regular sequence if and only if the solution set of the equations $\theta_{1}=0, \ldots, \theta_{n}=0$ in $\mathbb{C}^{n}$ consists only of the origin $\{0\}$. (This is because the above characterization (2) is valid for any coefficient field and hence [15, Proposition 5.1] gives the claim.)

The following simple fact, which follows from [45, Chapter 1, Theorem 5.9], is also useful.

Lemma 6.3. Let $g_{1}, \ldots, g_{n}$ be positive-degree homogeneous polynomials in $\mathbb{Q}\left[x_{1}, \ldots, x_{n}\right]$. Suppose $\left\{g_{1}, \ldots, g_{n}\right\}$ is a regular sequence in $\mathbb{Q}\left[x_{1}, \ldots, x_{n}\right]$. Then $\mathbb{Q}\left[x_{1}, \ldots, x_{n}\right]$ is finitely generated as a $\mathbb{Q}\left[g_{1}, \ldots, g_{n}\right]$-module.

With these preliminaries in place, we begin our computation of the Hilbert series $F\left(H_{S}^{*}(\operatorname{Hess}(N, h)), s\right)$ of the equivariant cohomology ring $H_{S}^{*}(\operatorname{Hess}(\mathrm{N}, h))$. Our first step towards this goal is to compute the Hilbert series of the ordinary cohomology ring $H^{*}(\operatorname{Hess}(\mathrm{N}, h))$ using results of Mbirika 33] (we also took inspiration from related work of Peterson and Brion-Carrell as in [7]).

Lemma 6.4. Let $n$ be a positive integer and $h:[n] \rightarrow[n]$ a Hessenberg function. Let Hess $(\mathrm{N}, h)$ denote the corresponding regular nilpotent Hessenberg variety. Then the Hilbert series of the ordinary cohomology ring $H^{*}(\operatorname{Hess}(\mathrm{N}, h))$ (equipped with the usual grading) is

$$
F\left(H^{*}(\operatorname{Hess}(\mathrm{N}, h)), s\right)=\prod_{j=1}^{n} \frac{1-s^{2(h(j)-j+1)}}{1-s^{2}} .
$$

The following proof of Lemma 6.4 uses a trick which re-writes certain expressions as a product over negative roots $\mathrm{NR}(h)$ contained in the Hessenberg space as in (2.4).

Proof of Lemma 6.4. Following [33], we define integers $\beta_{i}$ for $i \in[n]$ by

$$
\beta_{i}:=i-|\{k \in[n] \mid h(k)<i\}| .
$$

It is straightforward to see that $\beta_{i}-1$ is the number of elements in $\operatorname{NR}(h)$ which are contained in the $i$-th row, i.e. pairs in $\operatorname{NR}(h)$ whose first coordinates equal to $i$. In particular, $\beta_{i}>0$ for all $i \in[n]$. For a positive integer $\beta$, denote by $h_{\beta}\left(x_{1}, \ldots, x_{k}\right)$ the degree- $\beta$ complete symmetric polynomial in the listed variables. Following [33] we define $J_{h}$ to be the ideal in $\mathbb{Q}\left[x_{1}, \ldots, x_{n}\right]$ generated by the polynomials $h_{\beta_{n}}\left(x_{n}\right), h_{\beta_{n-1}}\left(x_{n-1}, x_{n}\right), \ldots, h_{\beta_{1}}\left(x_{1}, \ldots, x_{n}\right)$. It turns out [33, Theorem 3.4.3] that the Hilbert series of $H^{*}(\operatorname{Hess}(\mathrm{N}, h))$ and $\mathbb{Q}\left[x_{1}, \ldots, x_{n}\right] / J_{h}$ coincide:

$$
F\left(H^{*}(\operatorname{Hess}(\mathrm{N}, h)), s\right)=F\left(\mathbb{Q}\left[x_{1}, \ldots, x_{n}\right] / J_{h}, s\right) .
$$

We next claim that this sequence $h_{\beta_{n}}\left(x_{n}\right), h_{\beta_{n-1}}\left(x_{n-1}, x_{n}\right), \ldots, h_{\beta_{1}}\left(x_{1}, \ldots, x_{n}\right)$ forms a regular sequence. Since there are precisely $n$ elements in the sequence, which is equal to the number of variables in the ambient polynomial ring, we may use the characterization in Remark 6.2 (3) above; in particular, it suffices to see that their common zero locus in $\mathbb{C}^{n}$ is just the origin $0 \in \mathbb{C}^{n}$. Noting as above that each $\beta_{i}$ is positive, we see first that $h_{\beta_{n}}\left(x_{n}\right)=x_{n}^{\beta_{n}}=0$ implies $x_{n}=0$. But if $x_{n}=0$ then $h_{\beta_{n-1}}\left(x_{n}, x_{n-1}\right)=h_{\beta_{n-1}}\left(0, x_{n-1}\right)=$ $h_{\beta_{n-1}}\left(x_{n-1}\right)=x_{n-1}^{\beta_{n-1}}=0$ and we may conclude $x_{n-1}=0$. Continuing in this manner we see that all $x_{i}=0$, i.e. the common zero locus is $\{0\}$ as desired. Using the characterization of regular sequences in Remark 6.2(2) we then have

$$
F\left(\mathbb{Q}\left[x_{1}, \ldots, x_{n}\right] / J_{h}, s\right)=F\left(\mathbb{Q}\left[x_{1}, \ldots, x_{n}\right], s\right) \prod_{i=1}^{n}\left(1-s^{2 \beta_{i}}\right)=\prod_{i=1}^{n} \prod_{k=1}^{\beta_{i}-1} \frac{1-s^{2(k+1)}}{1-s^{2 k}} .
$$


As we already observed, $\beta_{i}-1$ counts the number of pairs $(i, j)$ in $\mathrm{NR}(h)$ in the $i$-th row. Put another way, the set of pairs in $\operatorname{NR}(h)$ with first coordinate equal to $i$ can also be expressed as $\left\{(i, i-k) \mid 1 \leq k \leq \beta_{i}-1\right\}$ and in particular we see that the differences $i-(i-k)=k$ of the coordinates range precisely between 1 and $\beta_{i}-1$. Using the same reasoning for each $i \in[n]$, the last expression in (6.1) can be re-written as

$$
\prod_{(i, j) \in \mathrm{NR}(h)} \frac{1-s^{2(\mathrm{ht}(i, j)+1)}}{1-s^{2 \cdot \mathrm{ht}(i, j)}}
$$

where $\operatorname{ht}(i, j):=i-j$ is called the height $3^{3}$. But now we may decompose the terms in (6.2) according to columns instead of rows. In this case, from the definition of $\mathrm{NR}(h)$ it is straightforward to rewrite (6.2) as

$$
\prod_{j=1}^{n} \prod_{k=1}^{h(j)-j} \frac{1-s^{2(k+1)}}{1-s^{2 k}}=\prod_{j=1}^{n} \frac{1-s^{2(h(j)-j+1)}}{1-s^{2}} .
$$

This proves the claim.

We now wish to relate the Hilbert series of $H^{*}(\operatorname{Hess}(\mathrm{N}, h))$ to the Hilbert series of the quotient ring $\mathbb{Q}\left[x_{1}, \ldots, x_{n}\right] / \check{I}_{h}$. This will in turn allow us to compute and compare the Hilbert series of $H_{S}^{*}(\operatorname{Hess}(\mathrm{N}, h))$ and $\mathbb{Q}\left[x_{1}, \ldots, x_{n}, t\right] / I_{h}$. However, in order to accomplish this, we must first analyze the relationship between the series $f_{i, j}$ defined in Section 3 and the series $\check{f}_{i, j} \in \mathbb{Q}\left[x_{1}, \ldots, x_{n}\right]$ defined in (1.2). It turns out that $\check{f}_{i, j}$ is obtained from $f_{i, j}$ by setting the variable $t$ equal to 0 .

Lemma 6.5. For all $n \geq i \geq j \geq 1$, we have

$$
f_{i, j}\left(x_{1}, \ldots, x_{n}, t=0\right)=\check{f}_{i, j}=\sum_{k=1}^{j}\left(x_{k} \prod_{\ell=j+1}^{i}\left(x_{k}-x_{\ell}\right)\right) .
$$

Proof. The second equality is just the definition (1.2), so we only need to prove the first equality. Let $f_{i, j}^{\prime}:=f_{i, j}\left(x_{1}, \ldots, x_{n}, 0\right) \in \mathbb{Q}\left[x_{1}, \ldots, x_{n}\right]$. We wish to show $f_{i, j}^{\prime}=\check{f}_{i, j}$. From Definition 3.1 we immediately see that these polynomials satisfy the following recursion relations:

$$
\begin{aligned}
& f_{j, j}^{\prime}=\sum_{k=1}^{j} x_{k} \quad \text { for } j \in[n], \\
& f_{i, j}^{\prime}=f_{i-1, j-1}^{\prime}+\left(x_{j}-x_{i}\right) f_{i-1, j}^{\prime} \quad \text { for } n \geq i>j \geq 1 .
\end{aligned}
$$

We introduce a total order on the set $\{(i, j) \in[n] \times[n] \mid i \geq j\}$ by the condition

$$
\left(i^{\prime}, j^{\prime}\right) \leq(i, j) \quad \text { if and only if } \quad\left(i^{\prime}-j^{\prime}<i-j\right) \text { or }\left(i^{\prime}-j^{\prime}=i-j \text { and } i^{\prime} \leq i\right)
$$

and we prove the claim by induction on this total order. When $i=j$, it is clear by (6.4) that the claim holds. Let $i>j$ and assume the claim holds for $\left(i^{\prime}, j^{\prime}\right)$ less than $(i, j)$. Since we have $(i-1, j-1)<(i, j)$ and $(i-1, j)<(i, j)$ by definition of our total order, the inductive hypothesis and (6.4) show that

$$
\begin{aligned}
f_{i, j}^{\prime} & =f_{i-1, j-1}^{\prime}+\left(x_{j}-x_{i}\right) f_{i-1, j}^{\prime} \\
& =\sum_{k=1}^{j-1}\left(x_{k} \prod_{\ell=j}^{i-1}\left(x_{k}-x_{\ell}\right)\right)+\left(x_{j}-x_{i}\right) \sum_{k=1}^{j}\left(x_{k} \prod_{\ell=j+1}^{i-1}\left(x_{k}-x_{\ell}\right)\right) \\
& =\sum_{k=1}^{j-1}\left(x_{k} \prod_{\ell=j+1}^{i-1}\left(x_{k}-x_{\ell}\right)\right)\left(\left(x_{k}-x_{j}\right)+\left(x_{j}-x_{i}\right)\right)+\left(x_{j}-x_{i}\right) x_{j} \prod_{\ell=j+1}^{i-1}\left(x_{j}-x_{\ell}\right) \\
& =\sum_{k=1}^{j-1}\left(x_{k} \prod_{\ell=j+1}^{i}\left(x_{k}-x_{\ell}\right)\right)+x_{j} \prod_{\ell=j+1}^{i}\left(x_{j}-x_{\ell}\right)=\sum_{k=1}^{j}\left(x_{k} \prod_{\ell=j+1}^{i}\left(x_{k}-x_{\ell}\right)\right)=\check{f}_{i, j}
\end{aligned}
$$

as desired.

\footnotetext{
${ }^{3}$ Here, contrary to customary usage, we require that the height of a negative root is a positive integer.
} 
For future reference, we also record the degrees of the polynomials $f_{i, j}$ and $\check{f}_{i, j}$, both of which are immediate from their definitions.

Lemma 6.6. The degree of $f_{i, j}$ and $\check{f}_{i, j}$ in the variables $x_{i}$ and $t$ is $i-j+1$. With respect to the grading in $\mathbb{Q}\left[x_{1}, \ldots, x_{n}, t\right]$ and $\mathbb{Q}\left[x_{1}, \ldots, x_{n}\right]$ respectively, we have

$$
\operatorname{deg}\left(f_{i, j}\right)=\operatorname{deg}\left(\check{f}_{i, j}\right)=2(i-j+1) .
$$

We denote by

$$
\check{I_{h}}:=\left(\check{f}_{h(j), j} \mid 1 \leq j \leq n\right) \subset \mathbb{Q}\left[x_{1}, \ldots, x_{n}\right]
$$

the ideal of $\mathbb{Q}\left[x_{1}, \ldots, x_{n}\right]$ generated by the polynomials $\check{f}_{h(j), j}$. Our next goal is to relate the polynomials $\check{f}_{h(j), j}$ (and the ideal $\check{I}_{h}$ they generate) with the Hilbert series $F\left(H^{*}(\operatorname{Hess}(\mathrm{N}, h)), s\right)$. Armed with Lemma 6.4 and the results summarized in Remark [6.2, we can accomplish this goal once we show that the $\check{f}_{h(j), j}$ form a regular sequence, which we do in the next two lemmas.

Lemma 6.7. ([17, Exercise 1, page 74]) Let $m$ be an arbitrary positive integer and $\mathrm{y}_{1}, \ldots, \mathrm{y}_{m}$ be indeterminates. For $i$ a positive integer let $e_{i}(\mathrm{y}):=\sum_{1 \leq k_{1}<\cdots<k_{i} \leq m} \mathrm{y}_{k_{1}} \cdots \mathrm{y}_{k_{i}}$ and $\mathrm{p}_{i}(\mathrm{y}):=\sum_{k=1}^{m} \mathrm{y}_{k}^{i}$ be the $i$-th elementary symmetric polynomial and the $i$-th power sum respectively. Then we have

$$
-\sum_{r=1}^{q-1}(-1)^{r} e_{r}(\mathrm{y}) \mathrm{p}_{q-r}(\mathrm{y})=(-1)^{q} q e_{q}(\mathrm{y})+\mathrm{p}_{q}(\mathrm{y}) \quad \text { for any } q \geq 1 .
$$

Lemma 6.8. The polynomials $\check{f}_{h(1), 1}, \check{f}_{h(2), 2}, \cdots, \check{f}_{h(n), n}$ form a regular sequence in $\mathbb{Q}\left[x_{1}, \ldots, x_{n}\right]$.

Proof. We use Remark 6.2(3) to prove this claim, that is, we show that the solution set in $\mathbb{C}^{n}$ of the equations $\check{f}_{h(j), j}=0$ for all $j \in[n]$ consists of only the origin $\{0\}$. Observe that if $\breve{f}_{h(j), j}=0$ for all $j \in[n]$ then from Lemma 4.1 we also have $\check{f}_{n, j}=0$ for all $j \in[n]$ since we have (6.3) and the substitution $t=0$ is a ring homomorphism from $\mathbb{Q}\left[x_{1}, \ldots, x_{n}, t\right]$ to $\mathbb{Q}\left[x_{1}, \ldots, x_{n}\right]$. Hence it suffices to prove the statement of the lemma in the special case when $h(j)=n$ for all $j \in[n]$, i.e. that if $\check{f}_{n, j}=0$ for all $j \in[n]$ then $x_{j}=0$ for all $j \in[n]$.

To prove this, we first claim that for $j \in[n]$ we have

$$
\check{f}_{n, j}=\sum_{i=0}^{j-1}(-1)^{i} e_{i}\left(x_{n+2-j}, \ldots, x_{n}\right) \mathrm{p}_{j-i}(x)
$$

where we denote $\mathrm{p}_{j-i}(x)=\mathrm{p}_{j-i}\left(x_{1}, \ldots, x_{n}\right)$. This equality holds since from (6.3) and (6.7) the LHS is

$$
\begin{aligned}
\check{f}_{n, j} & =\sum_{k=1}^{n+1-j}\left(x_{k} \prod_{\ell=n+2-j}^{n}\left(x_{k}-x_{\ell}\right)\right) \\
& =\sum_{k=1}^{n+1-j} x_{k}\left(\sum_{i=0}^{j-1}(-1)^{i} e_{i}\left(x_{n+2-j}, \ldots, x_{n}\right) x_{k}^{j-1-i}\right) \\
& =\sum_{i=0}^{j-1}(-1)^{i} e_{i}\left(x_{n+2-j}, \ldots, x_{n}\right) \mathrm{p}_{j-i}\left(x_{1}, \ldots, x_{n+1-j}\right) \\
& =\mathrm{p}_{j}\left(x_{1}, \ldots, x_{n+1-j}\right)+\sum_{i=1}^{j-1}(-1)^{i} e_{i}\left(x_{n+2-j}, \ldots, x_{n}\right)\left(\mathrm{p}_{j-i}(x)-\mathrm{p}_{j-i}\left(x_{n+2-j}, \ldots, x_{n}\right)\right) \\
& =\mathrm{p}_{j}\left(x_{1}, \ldots, x_{n+1-j}\right)+\mathrm{p}_{j}\left(x_{n+2-j}, \ldots, x_{n}\right)+\sum_{i=1}^{j-1}(-1)^{i} e_{i}\left(x_{n+2-j}, \ldots, x_{n}\right) \mathrm{p}_{j-i}(x)
\end{aligned}
$$

by (6.7) and $e_{j}\left(x_{n+2-j}, \ldots, x_{n}\right)=0$

$=\sum_{i=0}^{j-1}(-1)^{i} e_{i}\left(x_{n+2-j}, \ldots, x_{n}\right) \mathbf{p}_{j-i}(x)$. 
Now (6.8) shows that the transition matrix from $\mathrm{p}_{1}(x), \ldots, \mathrm{p}_{n}(x)$ to $\check{f}_{n, 1}, \ldots, \check{f}_{n, n}$ is lower-triangular with diagonal entries all equal to 1 , and hence the ideal of $\mathbb{Q}\left[x_{1}, \ldots, x_{n}\right]$ generated by $\check{f}_{n, 1}, \ldots, \check{f}_{n, n}$ and the ideal of $\mathbb{Q}\left[x_{1}, \ldots, x_{n}\right]$ generated by power sums $\mathrm{p}_{1}(x), \ldots, \mathrm{p}_{n}(x)$ are the same ;

$$
\left(\check{f}_{n, 1}, \ldots, \check{f}_{n, n}\right)=\left(\mathrm{p}_{1}(x), \ldots, \mathrm{p}_{n}(x)\right) \subset \mathbb{Q}\left[x_{1}, \ldots, x_{n}\right] .
$$

Recall that we assume that $\check{f}_{n, j}=0$ for all $j$ with $1 \leq j \leq n$. In particular, we obtain $\mathrm{p}_{j}(x)=0$ for all $j$ with $1 \leq j \leq n$. It is well-known and easy to prove that this implies that $x_{1}=\cdots=x_{n}=0$. Now the claim follows from the characterization of regular sequences in Remark 6.2(3).

A computation of the Hilbert series is now straightforward.

Corollary 6.9. The Hilbert series of the graded $\mathbb{Q}$-algebras $H^{*}(\operatorname{Hess}(\mathrm{N}, h))$ and $\mathbb{Q}\left[x_{1}, \ldots, x_{n}\right] / \check{I}_{h}$ are equal, i.e.

$$
F\left(H^{*}(\operatorname{Hess}(\mathrm{N}, h)), s\right)=F\left(\mathbb{Q}\left[x_{1}, \ldots, x_{n}\right] / \check{I}_{h}, s\right)=\prod_{j=1}^{n} \frac{1-s^{2(h(j)-j+1)}}{1-s^{2}} .
$$

Proof. Recalling that $\operatorname{deg} \check{f}_{h(j), j}=2(h(j)-j+1)$ from (6.5), Lemma 6.8 and Remark 6.2 (2) show that

$$
F\left(\mathbb{Q}\left[x_{1}, \ldots, x_{n}\right] / \check{I_{h}}, s\right)=\prod_{j=1}^{n} \frac{1-s^{2(h(j)-j+1)}}{1-s^{2}} .
$$

Thus, together with Lemma 6.4 we obtain the claim.

We now turn our attention to the main goal of this section, which is the computation of the Hilbert series $F\left(H_{S}^{*}(\operatorname{Hess}(\mathrm{N}, h)), s\right)$ in terms of the ideal $I_{h}$ generated by the polynomials $f_{h(j), j}$. We continue to use the technique of regular sequences. Indeed, our first step, Lemma 6.10 below, states that the $n+1$ homogeneous polynomials $\left\{f_{h(1), 1}, \ldots, f_{h(n), n}, t\right\}$ form a regular sequence in $\mathbb{Q}\left[x_{1}, \ldots, x_{n}, t\right]$; this in fact follows easily from the above arguments.

Lemma 6.10. The polynomials $f_{h(1), 1}, \ldots, f_{h(n), n}, t$ form a regular sequence in $\mathbb{Q}\left[x_{1}, \ldots, x_{n}, t\right]$. Moreover, $\mathbb{Q}\left[x_{1}, \ldots, x_{n}, t\right]$ is a finitely generated and free $\mathbb{Q}\left[f_{h(1), 1}, \ldots, f_{h(n), n}, t\right]$-module.

Proof. By Remark 6.2(3), the first claim follows from Lemma 6.5 and Lemma 6.8 The second claim then follows from the characterization in Remark 6.2(1) and Lemma 6.3.

As we have just seen, $\mathbb{Q}\left[x_{1}, \ldots, x_{n}, t\right]$ is a free and finitely generated $\mathbb{Q}\left[f_{h(1), 1}, \ldots, f_{h(n), n}, t\right]$-module. A straightforward argument (using, for instance, a choice of basis together with the fact that $f_{h(1), 1}, \ldots, f_{h(n), n}, t$ are algebraically independent over $\mathbb{Q})$ then shows that the quotient $\mathbb{Q}\left[x_{1}, \ldots, x_{n}, t\right] /\left(f_{h(1), 1}, \ldots, f_{h(n), n}\right)=$ $\mathbb{Q}\left[x_{1}, \ldots, x_{n}, t\right] / I_{h}$ is a free and finitely generated module over $\mathbb{Q}[t]$. We record the following.

Corollary 6.11. As $\mathbb{Q}[t]$-modules and hence as graded $\mathbb{Q}$-vector spaces, we have

$$
\mathbb{Q}\left[x_{1}, \ldots, x_{n}, t\right] / I_{h} \cong \mathbb{Q}[t] \otimes_{\mathbb{Q}}\left(\mathbb{Q}\left[x_{1}, \ldots, x_{n}\right] / \check{I}_{h}\right) .
$$

In particular,

$$
F\left(\mathbb{Q}\left[x_{1}, \ldots, x_{n}, t\right] / I_{h}, s\right)=\frac{F\left(\mathbb{Q}\left[x_{1}, \ldots, x_{n}\right] / \check{I_{h}}, s\right)}{1-s^{2}} .
$$

Proof. Since $\mathbb{Q}\left[x_{1}, \ldots, x_{n}, t\right] / I_{h}$ is a finitely generated free $\mathbb{Q}[t]$-module as observed above, we have

$$
\mathbb{Q}\left[x_{1}, \ldots, x_{n}, t\right] / I_{h} \cong \mathbb{Q}[t] \otimes \mathbb{Q}\left(\mathbb{Q}\left[x_{1}, \ldots, x_{n}, t\right] /\left(f_{h(1), 1}, \ldots, f_{h(n), n}, t\right)\right)
$$

as $\mathbb{Q}[t]$-modules. The module in the right hand side is naturally isomorphic to $\mathbb{Q}[t] \otimes_{\mathbb{Q}}\left(\mathbb{Q}\left[x_{1}, \ldots, x_{n}\right] / \check{I}_{h}\right)$ by the definition (6.6) of $\check{I}_{h}$. Hence, we obtain the first claim. In particular this means

$$
F\left(\mathbb{Q}\left[x_{1}, \ldots, x_{n}, t\right] / I_{h}, s\right)=F(\mathbb{Q}[t], s) F\left(\mathbb{Q}\left[x_{1}, \ldots, x_{n}\right] / \check{I}_{h}, s\right)=\frac{F\left(\mathbb{Q}\left[x_{1}, \ldots, x_{n}\right] / \check{I}_{h}, s\right)}{1-s^{2}}
$$

as desired.

The main result of this section now follows easily. 
Proposition 6.12. The Hilbert series of the graded $\mathbb{Q}$-algebras $H_{S}^{*}(\operatorname{Hess}(\mathrm{N}, h))$ and $\mathbb{Q}\left[x_{1}, \ldots, x_{n}, t\right] / I_{h}$ are equal, i.e. $F\left(\mathbb{Q}\left[x_{1}, \ldots, x_{n}, t\right] / I_{h}, s\right)=F\left(H_{S}^{*}(\operatorname{Hess}(\mathrm{N}, h)), s\right)$.

Proof. Since Hess $(\mathrm{N}, h)$ admits a paving by complex affines (cf. discussion before (2.13)), we have that $H_{S}^{*}(\operatorname{Hess}(\mathrm{N}, h)) \cong H_{S}^{*}(\mathrm{pt}) \otimes_{\mathbb{Q}} H^{*}(\operatorname{Hess}(\mathrm{N}, h))$ as $H_{S}^{*}(\mathrm{pt})$-modules and hence also as graded $\mathbb{Q}$-vector spaces. In particular,

$$
F\left(H_{S}^{*}(\operatorname{Hess}(\mathrm{N}, h)), s\right)=F\left(H_{S}^{*}(\mathrm{pt}), s\right) F\left(H^{*}(\operatorname{Hess}(\mathrm{N}, h)), s\right) .
$$

Also since $H_{S}^{*}(\mathrm{pt}) \cong \mathbb{Q}[t]$ is a polynomial ring in one variable we have $F\left(H_{S}^{*}(\mathrm{pt}), s\right)=\frac{1}{1-s^{2}}$ and we obtain

$$
F\left(H_{S}^{*}(\operatorname{Hess}(\mathrm{N}, h)), s\right)=\frac{F\left(H^{*}(\operatorname{Hess}(\mathrm{N}, h)), s\right)}{1-s^{2}}=\frac{F\left(\mathbb{Q}\left[x_{1}, \ldots, x_{n}\right] / \check{I}_{h}, s\right)}{1-s^{2}}=F\left(\mathbb{Q}\left[x_{1}, \ldots, x_{n}, t\right] / I_{h}, s\right)
$$

by Corollary 6.9 and Corollary 6.11 as desired.

\section{Second part of proof of Theorem 3.3 And proof of Theorem A}

The purpose of this section is to complete the proof of Theorem 3.3 and hence also of Theorem A. Specifically, we prove that the graded $\mathbb{Q}[t]$-algebra homomorphism

$$
\varphi_{h}: \mathbb{Q}\left[x_{1}, \ldots, x_{n}, t\right] / I_{h} \rightarrow H_{S}^{*}(\operatorname{Hess}(\mathrm{N}, h)) \quad ; \quad x_{i} \mapsto \bar{\tau}_{i}^{S}, \quad t \mapsto t
$$

(which was shown to be well-defined in Corollary 5.4) is in fact an isomorphism. Before launching into the proof we sketch the essential idea. As mentioned in the introductory remarks to Section 66, if two vector spaces are a priori known to have the same dimension, then a linear map between them is an isomorphism if and only if it is injective if and only if it is surjective. We will now use this elementary linear algebra fact to its full effect, given that we have shown that the dimensions of $H^{*}(\operatorname{Hess}(\mathrm{N}, h))$ and $\mathbb{Q}\left[x_{1}, \ldots, x_{n}\right] / \check{I}_{h}$ coincide (Corollary [6.9), and that the dimensions of (the graded pieces of) $H_{S}^{*}(\operatorname{Hess}(\mathrm{N}, h))$ and $\mathbb{Q}\left[x_{1}, \ldots, x_{n}, t\right] / I_{h}$ coincide (Proposition 6.12). The other essential trick we use is that of localization: instead of directly attacking the problem of showing that $\varphi_{h}$ is injective (which, as we said above, would suffice to show that $\varphi_{h}$ is an isomorphism), we show first that a certain localization $R^{-1} \varphi_{h}$ is an isomorphism by using the localization theorem in equivariant topology. We make this more precise below.

Let $R=\mathbb{Q}[t] \backslash\{0\}$ and consider the induced homomorphism $R^{-1} \varphi_{h}$ between the $R^{-1} \mathbb{Q}[t]$-algebras

$$
R^{-1} \varphi_{h}: R^{-1}\left(\mathbb{Q}\left[x_{1}, \ldots, x_{n}, t\right] / I_{h}\right) \rightarrow R^{-1} H_{S}^{*}(\operatorname{Hess}(\mathrm{N}, h)) .
$$

Recall from Section 2.1 that the $S$-equivariant cohomology of the full flag variety $\operatorname{Flag}\left(\mathbb{C}^{n}\right)$ is generated as an $H_{S}^{*}(\mathrm{pt})$-module by the $S$-equivariant first Chern classes of the tautological line bundles. In our setting, this means that for the special case $h=(n, n, \ldots, n)$, by the definition of $\varphi_{h}$ we already know that

$$
\mathbb{Q}\left[x_{1}, \ldots, x_{n}, t\right] \rightarrow H_{S}^{*}(\operatorname{Hess}(\mathrm{N}, h))=H_{S}^{*}\left(\operatorname{Flag}\left(\mathbb{C}^{n}\right)\right)
$$

is surjective. We harness this fact, together with the localization theorem in equivariant topology and our explicit description of the $S$-fixed point set of $\operatorname{Hess}(\mathrm{N}, h)$, to show that $R^{-1} \varphi_{h}$ is surjective for general $h$. The fact that $R^{-1} \varphi_{h}$ is an isomorphism then follows from a simple dimension-counting argument over the field $R^{-1} \mathbb{Q}[t]=\mathbb{Q}(t)$ of rational functions in one variable, as suggested in the introductory remarks above.

Lemma 7.1. The map $R^{-1} \varphi_{h}$ in (7.1) is an isomorphism.

Proof. For simplicity of notation in what follows, for the special case $h=(n, n, \ldots, n)$ with Hess $(\mathrm{N}, h)=$ $F \operatorname{lag}\left(\mathbb{C}^{n}\right)$, we denote the corresponding ideal by $I$ and the corresponding map by $\varphi$. Then, as discussed above, $\varphi$ is surjective. In particular, $R^{-1} \varphi$ is also surjective.

Next, recall from Lemma 4.1 that if we have $h \subset h^{\prime}$ for two Hessenberg functions $h$ and $h^{\prime}$ then $I_{h^{\prime}} \subset I_{h}$ and hence there exists a natural induced map $\mathbb{Q}\left[x_{1}, \ldots, x_{n}, t\right] / I_{h^{\prime}} \rightarrow \mathbb{Q}\left[x_{1}, \ldots, x_{n}, t\right] / I_{h}$. In our case, for any Hessenberg function $h$ it is always true that $h \subset h^{\prime}$ for the "largest" Hessenberg function $h^{\prime}:=(n, n, \ldots, n)$, so we conclude from Lemma 4.1 and a localization that there exists a natural map

$$
R^{-1}\left(\mathbb{Q}\left[x_{1}, \ldots, x_{n}, t\right] / I\right) \rightarrow R^{-1}\left(\mathbb{Q}\left[x_{1}, \ldots, x_{n}, t\right] / I_{h}\right) .
$$


In fact, we may enlarge this to the following commutative diagram

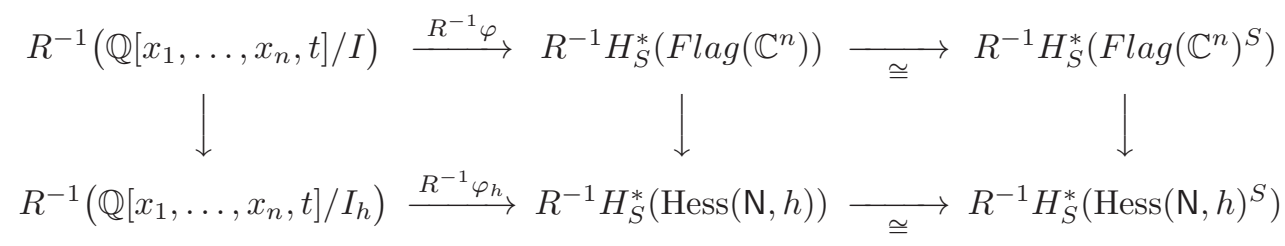

where the map in (7.2) is the leftmost vertical arrow, and all other unlabelled maps are induced from the geometric inclusion maps. The two horizontal maps in the square on the right are both isomorphisms by the localization theorem in equivariant topology [26, p.40]. Moreover, the right-most vertical map is a surjection since the $S$-fixed point set $\operatorname{Hess}(\mathrm{N}, h)^{S}$ is a subset of the $S$-fixed point set of Flag $\left(\mathbb{C}^{n}\right)$. Thus the middle vertical map must be surjective, and from there it also follows that $R^{-1} \varphi_{h}$ is surjective.

To show that $R^{-1} \varphi_{h}$ is in fact an isomorphism, we now compare the dimensions of $R^{-1}\left(\mathbb{Q}\left[x_{1}, \ldots, x_{n}, t\right] / I_{h}\right)$ and $R^{-1} H_{S}^{*}(\operatorname{Hess}(\mathrm{N}, h))$ as vector spaces over $R^{-1} \mathbb{Q}[t]=\mathbb{Q}(t)$ the field of rational functions in one variable $t$. Since we have $H_{S}^{*}(\operatorname{Hess}(N, h)) \cong H_{S}^{*}(\mathrm{pt}) \otimes_{\mathbb{Q}} H^{*}(\operatorname{Hess}(\mathrm{N}, h))$ by (2.13), we obtain

$$
R^{-1} H_{S}^{*}(\operatorname{Hess}(\mathrm{N}, h)) \cong R^{-1} H_{S}^{*}(\mathrm{pt}) \otimes_{\mathbb{Q}} H^{*}(\operatorname{Hess}(\mathrm{N}, h)) \cong \mathbb{Q}(t) \otimes_{\mathbb{Q}} H^{*}(\operatorname{Hess}(\mathrm{N}, h)) .
$$

In particular, the dimension of $R^{-1} H_{S}^{*}(\operatorname{Hess}(\mathrm{N}, h))$ as a $\mathbb{Q}(t)$-vector space is the dimension of $H^{*}(\operatorname{Hess}(\mathrm{N}, h))$ as a $\mathbb{Q}$-vector space. On the other hand, from Corollary 6.11 we also know that, as a $\mathbb{Q}[t]$-module, we have

$$
\mathbb{Q}\left[x_{1}, \ldots, x_{n}, t\right] / I_{h} \cong \mathbb{Q}[t] \otimes_{\mathbb{Q}}\left(\mathbb{Q}\left[x_{1}, \ldots, x_{n}\right] / \check{I}_{h}\right)
$$

which means that

$$
R^{-1}\left(\mathbb{Q}\left[x_{1}, \ldots, x_{n}, t\right] / I_{h}\right) \cong \mathbb{Q}(t) \otimes_{\mathbb{Q}}\left(\mathbb{Q}\left[x_{1}, \ldots, x_{n}\right] / \check{I}_{h}\right)
$$

and hence the dimension of $R^{-1}\left(\mathbb{Q}\left[x_{1}, \ldots, x_{n}, t\right] / I_{h}\right)$ as a $\mathbb{Q}(t)$-vector space is the dimension of $\mathbb{Q}\left[x_{1}, \ldots, x_{n}\right] / \check{I_{h}}$ as a $\mathbb{Q}$-vector space. But we have just seen in Corollary 6.9 that these two $\operatorname{rings} H^{*}(\operatorname{Hess}(\mathrm{N}, h))$ and $\mathbb{Q}\left[x_{1}, \ldots, x_{n}\right] / \check{I}_{h}$ have the same Hilbert series, and in particular are of the same dimension. Since $R^{-1} \varphi_{h}$ has been shown to be a surjective map between vector spaces of the same dimension, it must be an isomorphism, as desired.

We now wish to use the fact that $R^{-1} \varphi_{h}$ is an isomorphism to deduce that $\varphi_{h}$ must be an isomorphism.

Proof of Theorem 3.3 Consider the following commutative diagram:

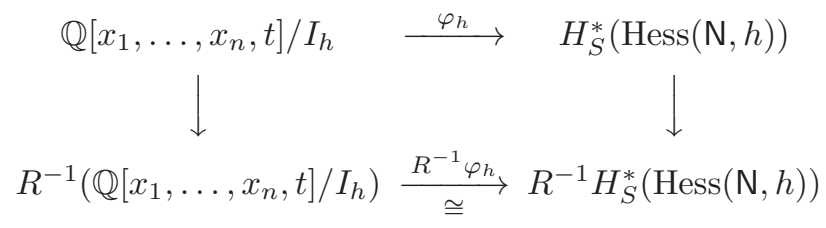

The left vertical map is injective since $\mathbb{Q}\left[x_{1}, \ldots, x_{n}, t\right] / I_{h}$ is a free $\mathbb{Q}[t]$-module by Corollary 6.11 We just saw that the bottom horizontal map is an isomorphism in Lemma 7.1. From the commutativity of the diagram we may conclude that $\varphi_{h}$ is an injection. But by Proposition 6.12 we know that the Hilbert series of the target and the domain agree, showing that their graded pieces have the same dimension. Thus, $\varphi_{h}$ is an isomorphism, as desired.

Finally, this implies that the $S$-equivariant cohomology ring $H_{S}^{*}(\operatorname{Hess}(\mathrm{N}, h))$ is generated by the first Chern class $\bar{\tau}_{i}^{S}$ of the $i$-th tautological line bundle (over Flag $\left(\mathbb{C}^{n}\right)$ ) restricted to Hess $(\mathrm{N}, h)$, and hence the restriction map $H_{T}^{*}\left(\operatorname{Flag}\left(\mathbb{C}^{n}\right)\right) \rightarrow H_{S}^{*}(\operatorname{Hess}(\mathrm{N}, h))$ is surjective.

We can now prove Theorem A. Indeed, since the odd degree cohomology groups of $\operatorname{Hess}(\mathrm{N}, h)$ vanish as discussed in Section 2, by setting $t=0$ we obtain the ordinary cohomology. Recall from Lemma 6.5 we have

$$
\check{f}_{i, j}=\left.f_{i, j}\left(x_{1}, \ldots, x_{n}, t\right)\right|_{t=0}=\sum_{k=1}^{j}\left(x_{k} \prod_{\ell=j+1}^{i}\left(x_{k}-x_{\ell}\right)\right) \quad(n \geq i \geq j \geq 1) .
$$


Let

$$
\bar{\tau}_{i} \in H^{2}(\operatorname{Hess}(\mathrm{N}, h))
$$

be the image of the Chern class $\tau_{i} \in H^{2}\left(F \operatorname{lag}\left(\mathbb{C}^{n}\right)\right)$ under the restriction map $H^{*}\left(F \operatorname{lag}\left(\mathbb{C}^{n}\right)\right) \rightarrow H^{*}(\operatorname{Hess}(\mathrm{N}, h))$. That is, $\bar{\tau}_{i}$ is the first Chern class of the tautological line bundle over Flag $\left(\mathbb{C}^{n}\right)$ restricted to Hess $(\mathrm{N}, h)$. (Its equivariant version $\bar{\tau}_{i}^{S} \in H_{S}^{2}(\operatorname{Hess}(\mathrm{N}, h))$ is defined in (3.2).)

Proof of Theorem A. Consider the forgetful map $H_{S}^{*}(\operatorname{Hess}(\mathrm{N}, h)) \rightarrow H^{*}(\operatorname{Hess}(\mathrm{N}, h))$ which sends the ideal of $H_{S}^{*}(\operatorname{Hess}(\mathrm{N}, h))$ generated by $t$ to zero. This map is surjective since Hess $(\mathrm{N}, h)$ admits a paving by complex affines [48 as mentioned in Section 2 and hence the Serre spectral sequence collapses at $E_{2}$-stage [35. Chapter III, Theorem 2.10]. Thus, from Theorem 3.3 together with (6.3), we obtain

$$
\mathbb{Q}\left[x_{1}, \ldots, x_{n}\right] / \check{I}_{h} \cong H_{S}^{*}(\operatorname{Hess}(\mathrm{N}, h)) /(t) \cong H^{*}(\operatorname{Hess}(\mathrm{N}, h))
$$

by sending each $x_{i}$ to $\bar{\tau}_{i}$ defined above where $\check{I}_{h}=\left(\check{f}_{h(j), j} \mid 1 \leq j \leq n\right)$. Since the (equivariant) restriction map $H_{T}^{*}\left(F \operatorname{lag}\left(\mathbb{C}^{n}\right)\right) \rightarrow H_{S}^{*}(\operatorname{Hess}(\mathrm{N}, h))$ is surjective from Theorem 3.3. so is the restriction map $H^{*}\left(\operatorname{Flag}\left(\mathbb{C}^{n}\right)\right) \rightarrow H^{*}(\operatorname{Hess}(\mathrm{N}, h))$.

\section{The Equivariant COHOMOLOGy RINGS OF REgular SEMisimple HessenberG VARIETIES}

Our second main result, Theorem $\mathrm{B}$, relates the ordinary cohomology ring $H^{*}(\operatorname{Hess}(\mathrm{N}, h))$ of the regular nilpotent Hessenberg variety and the $\mathfrak{S}_{n}$-invariant subring $H^{*}(\operatorname{Hess}(\mathrm{S}, h))^{\mathfrak{S}_{n}}$ of the ordinary cohomology of the regular semisimple Hessenberg variety. In this section, we recall the definition of the $\mathfrak{S}_{n}$-action on the $T$-equivariant cohomology $H_{T}^{*}(\operatorname{Hess}(\mathrm{S}, h))$ - which then induces an $\mathfrak{S}_{n}$-action on $H^{*}(\operatorname{Hess}(\mathrm{S}, h))$ - where $T$ is the usual maximal torus defined in (2.7). It is this $\mathfrak{S}_{n}$-action with respect to which we take the invariants in our Theorem B. We also record some preliminary results concerning this action.

Let $h \in H_{n}$ be a Hessenberg function and Hess $(\mathrm{S}, h)$ the regular semisimple Hessenberg variety associated to $h$ as defined in (1.5). Here and below, we use the notation

$$
d:=\operatorname{dim}_{\mathbb{C}} \operatorname{Hess}(\mathrm{S}, h)
$$

where the computation of this quantity in terms of the Hessenberg function $h$ is described in (2.6).

As we saw in Section 2 the maximal torus $T$ of $\operatorname{GL}(n, \mathbb{C})$ acts on $\operatorname{Flag}\left(\mathbb{C}^{n}\right)$ preserving Hess $(\mathrm{S}, h)$. It is also straightforward to see that

$$
\operatorname{Hess}(\mathrm{S}, h)^{T}=\operatorname{Flag}\left(\mathbb{C}^{n}\right)^{T} \cong \mathfrak{S}_{n} .
$$

Tymoczko described the equivariant cohomology ring $H_{T}^{*}(\operatorname{Hess}(\mathrm{S}, h))$ as an algebra over $H^{*}(B T)$ by using techniques introduced by Goresky, Kottwitz, and MacPherson [20, also called GKM theory. For details we refer to [49] and below we only state the facts relevant for our situation. Any Hessenberg variety (in Lie type A) admits a paving by complex affines (48, Theorem 7.1]), and hence the localization theorem of torus-equivariant topology implies that the inclusion map of the fixed point set induces an injection

$$
\iota_{3}: H_{T}^{*}(\operatorname{Hess}(\mathrm{S}, h)) \hookrightarrow H_{T}^{*}\left(\operatorname{Hess}(\mathrm{S}, h)^{T}\right) \cong \bigoplus_{w \in \mathfrak{S}_{n}} \mathbb{Q}\left[t_{1}, \ldots, t_{n}\right] .
$$

where we identify $\operatorname{Hess}(\mathrm{S}, h)^{T}=\operatorname{Flag}\left(\mathbb{C}^{n}\right)^{T} \cong \mathfrak{S}_{n}$ as above. For $\left.\alpha \in H_{T}^{*}(\operatorname{Hess}(\mathrm{S}, h))\right)$, since $\iota_{3}$ is injective, by abuse of notation we denote also by $\alpha$ its image in the RHS. In particular we denote by $\alpha(w) \in \mathbb{Q}\left[t_{1}, \ldots, t_{n}\right]$ the $w$-th component of $\alpha$ in the decomposition above.

In this setting, GKM theory yields the following concrete description of the image of $\iota_{3}$ [49]:

$$
H_{T}^{*}(\operatorname{Hess}(\mathrm{S}, h)) \cong\left\{\begin{array}{l|c}
\alpha \in \bigoplus_{w \in \mathfrak{S}_{n}} \mathbb{Q}\left[t_{1}, \ldots, t_{n}\right] & \begin{array}{c}
\alpha(w)-\alpha\left(w^{\prime}\right) \text { is divisible by } t_{w(i)}-t_{w(j)} \\
\text { if there exist } 1 \leq j<i \leq n \text { satisfying } \\
w^{\prime}=w(j i) \text { and } i \leq h(j)
\end{array}
\end{array}\right\}
$$

where $(j i) \in \mathfrak{S}_{n}$ denotes the element of $\mathfrak{S}_{n}$ which transposes $i$ and $j$. We call the condition described in the right hand side of (8.2) the GKM condition (for $\operatorname{Hess}(\mathrm{S}, h)$ ).

Note that if the Hessenberg function $h$ is chosen to be $h=(n, n, \ldots, n)$, then the corresponding Hessenberg variety $\operatorname{Hess}(\mathrm{S}, h)$ is equal to Flag $\left(\mathbb{C}^{n}\right)$. Since the condition $i \leq h(j)=n$ is always satisfied, the corresponding 
presentation of $H_{T}^{*}\left(F \operatorname{lag}\left(\mathbb{C}^{n}\right)\right)$ imposes more conditions on a collection $(\alpha(w))_{w \in \mathfrak{S}_{n}}$ than the RHS of (8.2). From this we can see that for an arbitrary Hessenberg function $h$, the restriction map

$$
H_{T}^{*}\left(\operatorname{Flag}\left(\mathbb{C}^{n}\right)\right) \rightarrow H_{T}^{*}(\operatorname{Hess}(\mathrm{S}, h))
$$

is an injection by considering the following commutative diagram

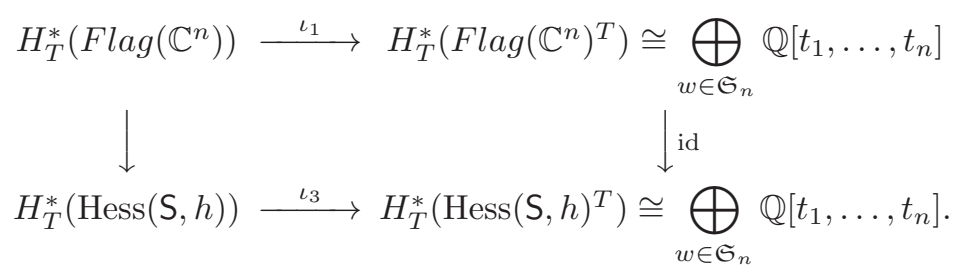

It turns out that the image of $t_{i} \in H_{T}^{*}(\mathrm{pt})=H^{*}(B T) \cong \mathbb{Q}\left[t_{1}, \ldots, t_{n}\right]$ under the composition

$$
H_{T}^{*}(\mathrm{pt}) \rightarrow H_{T}^{*}(\operatorname{Hess}(\mathrm{S}, h)) \stackrel{\iota 3}{\rightarrow} H_{T}^{*}\left(\operatorname{Hess}(\mathrm{S}, h)^{T}\right)
$$

is given simply by attaching the monomial $t_{i}$ to each vertex in the graph (see also (2.18)). Henceforth, by slight abuse of notation, we denote also by $t_{i}$ the equivariant cohomology class in $H_{T}^{*}(\operatorname{Hess}(\mathrm{S}, h))$ obtained in this way. In particular it is immediate, with this notation, that

$$
t_{i}(w)=t_{i} \text { for all } w \in \mathfrak{S}_{n} .
$$

This class $t_{i} \in H_{T}^{2}(\operatorname{Hess}(\mathrm{S}, h))$ is in fact the image of $t_{i} \in H^{2}(B T)$ (defined after (2.8)) under the canonical homomorphism $H^{*}(B T) \rightarrow H_{T}^{*}(\operatorname{Hess}(\mathrm{S}, h))$.

We now describe the $\mathfrak{S}_{n}$-action on $H_{T}^{*}(\operatorname{Hess}(\mathrm{S}, h))$ constructed explicitly by Tymoczko 49 . (This action also induces an $\mathfrak{S}_{n}$-action on the ordinary cohomology $H^{*}(\operatorname{Hess}(\mathrm{S}, h))$, as we explain below.) First, define an $\mathfrak{S}_{n}$-action on the polynomial ring $\mathbb{Q}\left[t_{1}, \ldots, t_{n}\right]$ in the standard way by permuting the indices of the variables, i.e. for $t_{i} \in \mathbb{Q}\left[t_{1}, \ldots, t_{n}\right]$ and $v \in \mathfrak{S}_{n}$ we define $v \cdot t_{i}:=t_{v(i)}$. This induces an $\mathfrak{S}_{n}$-action on $\mathbb{Q}\left[t_{1}, \ldots, t_{n}\right]$ by $\mathbb{Q}$-linear ring homomorphisms. Recall that by (8.2) the data of an element $\alpha \in H_{T}^{*}(\operatorname{Hess}(\mathrm{S}, h))$ is equivalent to a list $(\alpha(w))_{w \in \mathfrak{S}_{n}}$ of polynomials in $\mathbb{Q}\left[t_{1}, \ldots, t_{n}\right]$ satisfying the GKM conditions. With this understanding, Tymoczko defines, for $v \in \mathfrak{S}_{n}$ and $\alpha=(\alpha(w))_{w \in \mathfrak{S}_{n}}$, the element $v \cdot \alpha$ by the formula

$$
(v \cdot \alpha)(w):=v \cdot \alpha\left(v^{-1} w\right) \text { for all } w \in \mathfrak{S}_{n} .
$$

It is also straightforward to check that the class $v \cdot \alpha$ thus defined again satisfies the GKM conditions and hence this action is well-defined. It is straightforward to check that the "constant" class $t_{i} \in H_{T}^{*}(\operatorname{Hess}(\mathrm{S}, h))$ corresponding to $t_{i} \in H^{*}(B T)$ described in (8.4) satisfies $v \cdot t_{i}=t_{v(i)}$ for $v \in \mathfrak{S}_{n}$.

The above lemma implies that the ideal of $H_{T}^{*}(\operatorname{Hess}(\mathrm{S}, h))$ generated by the classes $t_{1}, \ldots, t_{n}$ is preserved by Tymoczko's $\mathfrak{S}_{n}$-action. Since the odd degree cohomology of $\operatorname{Hess}(\mathrm{S}, h)$ vanishes, the forgetful map $H_{T}^{*}(\operatorname{Hess}(\mathrm{S}, h)) \rightarrow H^{*}(\operatorname{Hess}(\mathrm{S}, h))$ is surjective [35, Ch III, Theorem 2.10 and Theorem 4.2], and the kernel is precisely the ideal generated by the $t_{i}$. Thus, we obtain an isomorphism

$$
H^{*}(\operatorname{Hess}(\mathrm{S}, h)) \cong H_{T}^{*}(\operatorname{Hess}(\mathrm{S}, h)) /\left(t_{1}, \ldots, t_{n}\right)
$$

and the fact that the ideal $\left(t_{1}, \ldots, t_{n}\right)$ is $\mathfrak{S}_{n}$-invariant implies that the RHS, and hence also the LHS, has a well-defined $\mathfrak{S}_{n}$-action. The following is then straightforward from the definitions.

Lemma 8.1. The diagram

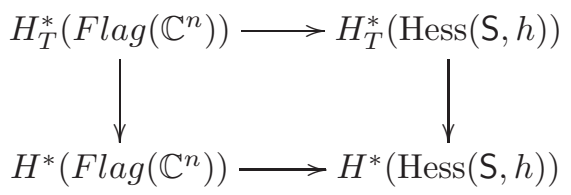

commutes, where the horizontal maps are induced from the inclusion map $\operatorname{Hess}(\mathrm{S}, h) \hookrightarrow$ Flag $\left(\mathbb{C}^{n}\right)$ and the vertical arrows are forgetful maps. Moreover, all maps in the diagram are $\mathfrak{S}_{n}$-equivariant.

Note that $\mathfrak{S}_{n}$ naturally acts on Flag $\left(\mathbb{C}^{n}\right)$ on the left by multiplication by permutation matrices, and it is well-known (see e.g. [30, [49]) that this induces Tymoczko's $\mathfrak{S}_{n}$-representation on $H_{T}^{*}\left(\right.$ Flag $\left.\left(\mathbb{C}^{n}\right)\right)$ and $H^{*}\left(F \operatorname{lag}\left(\mathbb{C}^{n}\right)\right)$. Note that this $\mathfrak{S}_{n}$-action is obtained by restricting the natural $\mathrm{GL}(n, \mathbb{C})$-action on Flag $\left(\mathbb{C}^{n}\right)$. 
The path-connectedness of $\mathrm{GL}(n, \mathbb{C})$ implies that the induced $\operatorname{GL}(n, \mathbb{C})$-representation on $H^{*}\left(\right.$ Flag $\left.\left(\mathbb{C}^{n}\right)\right)$ is trivial. Hence we obtain the following.

Lemma 8.2. ([49, Proposition 4.4]) The $\mathfrak{S}_{n}$-representation on $H^{*}\left(F l a g\left(\mathbb{C}^{n}\right)\right)$ is trivial.

\section{Properties of the $\mathfrak{S}_{n}$-ACtion on $H_{T}^{*}(\operatorname{Hess}(\mathrm{S}, h))$}

In this section, we prepare for the proof of Theorem B by analyzing in more detail the properties of the $\mathfrak{S}_{n}$-action on $H_{T}^{*}(\operatorname{Hess}(\mathrm{S}, h))$ defined in Section 8 . Our first result is Proposition 9.3 which explicitly identifies the $\mathfrak{S}_{n}$-invariant subring of $H_{T}^{*}\left(\operatorname{Hess}(\mathrm{S}, h)\right.$ ) (hence also of $H_{T}^{*}\left(F \operatorname{lag}\left(\mathbb{C}^{n}\right)\right)$ as a special case). Our second result is Proposition 9.5, which states that there exists an $\mathfrak{S}_{n}$-invariant non-degenerate pairing on the ordinary cohomology groups of complementary degree of $\operatorname{Hess}(\mathrm{S}, h)$.

We begin with Proposition 9.3 It will turn out that the $\mathfrak{S}_{n}$-invariant subring of $H_{T}^{*}(\operatorname{Hess}(\mathrm{S}, h)$ ) (and hence also $\left.H_{T}^{*}\left(F \operatorname{lag}\left(\mathbb{C}^{n}\right)\right)\right)$ is a copy of the polynomial $\operatorname{ring} H_{T}^{*}(\mathrm{pt}) \cong \mathbb{Q}\left[t_{1}, \ldots, t_{n}\right]$, but some care must be taken in defining the embedding of $H_{T}^{*}(\mathrm{pt})$ into $H_{T}^{*}\left(\operatorname{Hess}(\mathrm{S}, h)\right.$ ) (and $H_{T}^{*}\left(F \operatorname{lag}\left(\mathbb{C}^{n}\right)\right)$ ) that achieves this isomorphism. Specifically, the embedding does not take the element $t_{i} \in H_{T}^{*}(\mathrm{pt}) \cong \mathbb{Q}\left[t_{1}, \ldots, t_{n}\right]$ to the "constant class" in $H_{T}^{*}\left(F \operatorname{Flag}\left(\mathbb{C}^{n}\right)\right)$ (and in $\left.H_{T}^{*}(\operatorname{Hess}(\mathrm{S}, h))\right)$ described in (8.4) which takes the constant value $t_{i}(w)=t_{i}$ at all $w \in \mathfrak{S}_{n}$, as one might initially expect. Instead, the images are defined to be certain characteristic classes, as we now explain. Recall from (2.9) that $\tau_{i}^{T} \in H_{T}^{2}\left(F \operatorname{lag}\left(\mathbb{C}^{n}\right)\right)$ denotes the $T$-equivariant first Chern class of the tautological line bundle $E_{i} / E_{i-1}$ over $\operatorname{Flag}\left(\mathbb{C}^{n}\right)$. We denote by

$$
\hat{\tau}_{i}^{T} \in H_{T}^{2}(\operatorname{Hess}(\mathrm{S}, h))
$$

the image of $\tau_{i}^{T}$ under the restriction map $H_{T}^{*}\left(\operatorname{Flag}\left(\mathbb{C}^{n}\right)\right) \rightarrow H_{T}^{*}(\operatorname{Hess}(\mathrm{S}, h))$.

Lemma 9.1. Let $i \in[n]$. The classes $\tau_{i}^{T} \in H_{T}^{2}\left(\operatorname{Flag}\left(\mathbb{C}^{n}\right)\right)$ and $\hat{\tau}_{i}^{T} \in H_{T}^{2}(\operatorname{Hess}(\mathrm{S}, h))$ are $\mathfrak{S}_{n}$-invariant.

Proof. The following proof is independent of the choice of the Hessenberg function $h$, so since the choice $h=(n, n, \ldots, n)$ yields Hess $(\mathrm{S}, h)=F \operatorname{lag}\left(\mathbb{C}^{n}\right)$ as a special case, it suffices to show the claim for Hess $(\mathrm{S}, h)$. We have already seen from (2.19) that $\tau_{i}^{T}(w)=t_{w(i)}$. By the definition of $\hat{\tau}_{i}^{T}$ and the commutativity of (8.3), we also have

$$
\hat{\tau}_{i}^{T}(w)=t_{w(i)} .
$$

By (9.2) and the definition (8.5) of the $\mathfrak{S}_{n}$-action on $H_{T}^{*}(\operatorname{Hess}(\mathbf{S}, h))$, we can compute that for any $w \in \mathfrak{S}_{n}$ we have

$$
\left(v \cdot \hat{\tau}_{i}^{T}\right)(w)=v \cdot \hat{\tau}_{i}^{T}\left(v^{-1} w\right)=v \cdot t_{v^{-1} w(i)}=t_{v\left(v^{-1} w(i)\right)}=t_{w(i)}=\hat{\tau}_{i}^{T}(w)
$$

as desired.

We now define a graded $\mathbb{Q}$-algebra homomorphism $\Psi: H_{T}^{*}(\mathrm{pt}) \cong \mathbb{Q}\left[t_{1}, \ldots, t_{n}\right] \rightarrow H_{T}^{*}\left(\operatorname{Flag}\left(\mathbb{C}^{n}\right)\right)$ by sending the generator $t_{i}$ to the $i$-th equivariant Chern class $\tau_{i}^{T}$, and define $\widehat{\Psi}: H_{T}^{*}(\mathrm{pt}) \rightarrow H_{T}^{*}(\operatorname{Hess}(\mathrm{S}, h))$ by composing $\Psi$ with the natural restriction $H_{T}^{*}\left(F \operatorname{lag}\left(\mathbb{C}^{n}\right)\right) \rightarrow H_{T}^{*}(\operatorname{Hess}(\mathrm{S}, h))$. To discuss properties of $\Psi$ and $\widehat{\Psi}$, it is useful to observe that the invariant subspace $H_{T}^{*}(\operatorname{Hess}(\mathrm{S}, h))^{\mathfrak{S}_{n}}$ of $H_{T}^{*}(\operatorname{Hess}(\mathrm{S}, h)$ ) (respectively $H^{*}(\operatorname{Hess}(\mathrm{S}, h))^{\mathfrak{S}_{n}}$ of $\left.H^{*}(\operatorname{Hess}(\mathrm{S}, h))\right)$ in fact forms a subring.

Lemma 9.2. The symmetric group $\mathfrak{S}_{n}$ acts on $H_{T}^{*}(\operatorname{Hess}(\mathrm{S}, h))$ and $H^{*}(\operatorname{Hess}(\mathrm{S}, h))$ via ring automorphisms, i.e. for $v \in \mathfrak{S}_{n}$ and $\alpha, \beta \in H_{T}^{*}(\operatorname{Hess}(\mathrm{S}, h))$, we have $v \cdot(\alpha \beta)=(v \cdot \alpha)(v \cdot \beta)$, and similarly for $H^{*}(\operatorname{Hess}(\mathrm{S}, h))$. Moreover, the identity elements of the rings $H_{T}^{*}(\operatorname{Hess}(\mathrm{S}, h))$ and $H^{*}(\operatorname{Hess}(\mathrm{S}, h))$ are $\mathfrak{S}_{n}$-invariant.

Proof. Straightforward from the definition of the $\mathfrak{S}_{n}$-action on $H_{T}^{*}(\operatorname{Hess}(\mathrm{S}, h))$ and $H^{*}(\operatorname{Hess}(\mathrm{S}, h))$ given at (8.5) and (8.6), respectively.

By Lemma 9.1 the images of $\Psi$ and $\widehat{\Psi}$ are contained in the $\mathfrak{S}_{n}$-invariant subrings of $H_{T}^{*}\left(\right.$ Flag $\left.\left(\mathbb{C}^{n}\right)\right)$ and $H_{T}^{*}(\operatorname{Hess}(\mathrm{S}, h))$ respectively. In fact, we can say more.

Proposition 9.3. The $\mathbb{Q}$-algebra homomorphisms $\Psi$ and $\widehat{\Psi}$ induce isomorphisms from $H_{T}^{*}(\mathrm{pt}) \cong \mathbb{Q}\left[t_{1}, \ldots, t_{n}\right]$ to the subrings $H_{T}^{*}\left(F \operatorname{lag}\left(\mathbb{C}^{n}\right)\right)^{\mathfrak{S}_{n}}$ and $H_{T}^{*}(\operatorname{Hess}(\mathrm{S}, h))^{\mathfrak{S}_{n}}$ of $\mathfrak{S}_{n}$-invariants, respectively. In particular, the two subrings $H_{T}^{*}\left(\text { Flag }\left(\mathbb{C}^{n}\right)\right)^{\mathfrak{S}_{n}}$ and $H_{T}^{*}(\operatorname{Hess}(\mathrm{S}, h))^{\mathfrak{S}_{n}}$ are isomorphic. 
Proof. The proof we give below applies to $\operatorname{Hess}(\mathrm{S}, h)$ for any $h \in H_{n}$, thus includes $\operatorname{Flag}\left(\mathbb{C}^{n}\right)$ as a special case. In particular, showing that $\widehat{\Psi}: H_{T}^{*}(\mathrm{pt}) \rightarrow H_{T}^{*}(\operatorname{Hess}(\mathrm{S}, h))^{\mathfrak{S}_{n}}$ is an isomorphism for any $h \in H_{n}$ implies all the claims made in the proposition.

We first show injectivity of $\widehat{\Psi}$, for which it is useful to consider the projection $\pi_{e}: H_{T}^{*}\left(\operatorname{Hess}(\mathrm{S}, h)^{T}\right) \cong$ $\bigoplus_{w \in \mathfrak{S}_{n}} H_{T}^{*}(\mathrm{pt}) \rightarrow H_{T}^{*}(\mathrm{pt})$ of $\bigoplus_{w \in \mathfrak{S}_{n}} H_{T}^{*}(\mathrm{pt})$ to the component corresponding to the identity element $e \in$ $\mathfrak{S}_{n}$. Then, from the above computation $\hat{\tau}_{i}^{T}(w)=t_{w(i)}$ for $w \in \mathfrak{S}_{n}$, it follows that $\hat{\tau}_{i}^{T}(e)=t_{i}$ for all $i$ and hence the composition $H_{T}^{*}(\mathrm{pt}) \stackrel{\widehat{\Psi}}{\rightarrow} H_{T}^{*}(\operatorname{Hess}(\mathrm{S}, h))^{\mathfrak{S}_{n}} \stackrel{\pi_{e}}{\rightarrow} H_{T}^{*}(\mathrm{pt})$ is the identity map. In particular, $\widehat{\Psi}$ must be injective.

Next, observe that an $\left|\mathfrak{S}_{n}\right|$-tuple $\alpha=(\alpha(w))_{w \in \mathfrak{S}_{n}}$ is $\mathfrak{S}_{n}$-invariant if and only if $\alpha(w)=w \cdot \alpha(e)$ for all $w \in \mathfrak{S}_{n}$. Since the classes $\hat{\tau}_{i}^{T}$ satisfy both $\hat{\tau}_{i}^{T}(e)=t_{i}$ and $\hat{\tau}_{i}^{T}(w)=t_{w(i)}=w \cdot t_{i}=w \cdot \hat{\tau}_{i}^{T}(e)$ for all $w \in \mathfrak{S}_{n}$, it follows that any $\mathfrak{S}_{n}$-invariant $\left|\mathfrak{S}_{n}\right|$-tuple $\alpha=(\alpha(w))_{w \in \mathfrak{S}_{n}}$ can be written as a polynomial in the $\hat{\tau}_{i}^{T}$ : namely, if $\alpha(e)=F\left(t_{1}, \ldots, t_{n}\right) \in H_{T}^{*}(\mathrm{pt}) \cong \mathbb{Q}\left[t_{1}, \ldots, t_{n}\right]$, then $\alpha=F\left(\hat{\tau}_{1}^{T}, \ldots, \hat{\tau}_{n}^{T}\right)$. In particular, $\widehat{\Psi}$ is surjective, as desired.

Our second goal for this section is to show that there exists an $\mathfrak{S}_{n}$-invariant and non-degenerate pairing on the ordinary cohomology groups of $\operatorname{Hess}(\mathrm{S}, h)$ of complementary degree. This pairing is straightforward in the sense that it is essentially the usual Poincaré duality pairing, although care is needed since our variety Hess $(\mathrm{S}, h)$ need not be connected (it is, however, pure-dimensional [11); indeed, it is not hard to see that Hess $(\mathrm{S}, h)$ is disconnected if and only if $h(r)=r$ for some $r \in[n]$ (see [11, 47]). To see that the pairing is compatible with the $\mathfrak{S}_{n}$-action, we first work in $T$-equivariant cohomology and then deduce the desired results in ordinary cohomology.

We need some terminology. Recall from (8.1) that $d=\operatorname{dim}_{\mathbb{C}} \operatorname{Hess}(\mathrm{S}, h)$. Recall also that the collapsing map pr : Hess $(\mathrm{S}, h) \rightarrow$ pt induces a map

$$
\operatorname{pr}_{!}^{T}: H_{T}^{*}(\operatorname{Hess}(\mathrm{S}, h)) \rightarrow H_{T}^{*-2 d}(\mathrm{pt})=H^{*-2 d}(B T)
$$

often called the "equivariant integral" or "equivariant Gysin map". The equivariant integral is well-known to be an $H_{T}^{*}(\mathrm{pt})$-module homomorphism. Moreover, by the famous Atiyah-Bott-Berline-Vergne formula [3, 6] we may compute the equivariant integral by fixed point data as follows:

$$
\operatorname{pr}_{!}^{T}(\alpha)=\sum_{w \in \mathfrak{S}_{n}} \frac{\alpha(w)}{e_{w}}
$$

where $\alpha(w)$ denotes the restriction of $\alpha$ to the fixed point $w, e_{w}$ denotes the $T$-equivariant Euler class of the normal bundle to the fixed point $w$ in $\operatorname{Hess}(\mathrm{S}, h)$, and we have used our fixed identification $\operatorname{Hess}(\mathrm{S}, h)^{T}$ with $\mathfrak{S}_{n}$. Finally we recall that the equivariant and ordinary Gysin maps commute with forgetful maps;

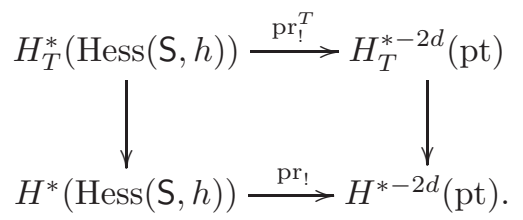

All the cohomology groups in the diagram (9.4) are equipped with $\mathfrak{S}_{n}$-actions, where the $\mathfrak{S}_{n}$-action on the ordinary cohomology $H^{*}(\mathrm{pt}) \cong \mathbb{Q}$ of a point is induced from that on $H_{T}^{*}(\mathrm{pt})$ by the isomorphism $H^{*}(\mathrm{pt}) \cong$ $H^{*}(B T) /\left(t_{1}, \ldots, t_{n}\right) \cong \mathbb{Q}\left[t_{1}, \ldots, t_{n}\right] /\left(t_{1}, \ldots, t_{n}\right) \cong \mathbb{Q}$. In particular, the forgetful map $H_{T}^{*}(\mathrm{pt}) \rightarrow H^{*}(\mathrm{pt})$ is $\mathfrak{S}_{n}$-equivariant by definition and the $\mathfrak{S}_{n}$-action on $H^{*}(\mathrm{pt})$ is trivial. We record the following.

Lemma 9.4. The ordinary Gysin map $\mathrm{pr}_{!}$in 9.4) is $\mathfrak{S}_{n}$-equivariant.

Proof. The definition given above of the $\mathfrak{S}_{n}$-action on $H^{*}(\mathrm{pt}) \cong \mathbb{Q}$ implies that the right vertical arrow in (9.4) is $\mathfrak{S}_{n}$-equivariant, and we saw in Lemma 8.1 that the left vertical arrow in (9.4) is $\mathfrak{S}_{n}$-equivariant. Recalling also that the left vertical arrow is surjective (see e.g. 8.6)), in order to prove the lemma, it therefore suffices to show that the top horizontal arrow $\operatorname{pr}_{!}^{T}$ is $\mathfrak{S}_{n}$-equivariant. Thus we wish to show

$$
\operatorname{pr}_{!}^{T}(v \cdot \alpha)=v \cdot \operatorname{pr}_{!}^{T}(\alpha)
$$


for $v \in \mathfrak{S}_{n}$ and $\alpha \in H_{T}^{*}(\operatorname{Hess}(\mathrm{S}, h))$. Before proceeding it is useful to observe that the $T$-equivariant Euler class $e_{w}$ of $\operatorname{Hess}(\mathrm{S}, h)$ at $w \in \mathfrak{S}_{n}$ is

$$
e_{w}=\prod_{j<i \leq h(j)}\left(t_{w(j)}-t_{w(i)}\right) \in H_{T}^{*}(\mathrm{pt})
$$

(e.g. [11]) where we have written $w=(w(1) w(2) \cdots w(n)) \in \mathfrak{S}_{n}$ in one-line notation. In particular, since $v w=(v w(1) v w(2) \cdots v w(n))$ in one-line notation, we conclude from the above that

$$
v \cdot e_{w}=v \cdot \prod_{j<i \leq h(j)}\left(t_{w(j)}-t_{w(i)}\right)=\prod_{j<i \leq h(j)}\left(t_{v w(j)}-t_{v w(i)}\right)=e_{v w}
$$

for any $v, w \in \mathfrak{S}_{n}$. Now, using the Atiyah-Bott-Berline-Vergne formula (9.3), we have

$$
\operatorname{pr}_{!}^{T}(v \cdot \alpha)=\sum_{w \in \mathfrak{S}_{n}} \frac{(v \cdot \alpha)(w)}{e_{w}}=\sum_{w \in \mathfrak{S}_{n}} \frac{v \cdot \alpha\left(v^{-1} w\right)}{e_{w}}=\sum_{u \in \mathfrak{S}_{n}} \frac{v \cdot \alpha(u)}{e_{v u}}=\sum_{u \in \mathfrak{S}_{n}} \frac{v \cdot \alpha(u)}{v \cdot e_{u}}=v \cdot \operatorname{pr}_{!}^{T}(\alpha)
$$

where the second equality follows from the definition (8.5) of the $\mathfrak{S}_{n}$-representation on $H_{T}^{*}(\operatorname{Hess}(\mathrm{S}, h))$ and the fourth equality follows from the above observation for $v \cdot e_{w}$. This proves the lemma.

We now define a pairing $\langle\cdot, \cdot\rangle$ on the ordinary cohomology $H^{*}(\operatorname{Hess}(\mathrm{S}, h))$ as follows: for $0 \leq k \leq d$, we define

$$
\langle\cdot, \cdot\rangle: H^{2 k}(\operatorname{Hess}(\mathrm{S}, h)) \times H^{2 d-2 k}(\operatorname{Hess}(\mathrm{S}, h)) \rightarrow \mathbb{Q} \cong H^{0}(\mathrm{pt}) \quad ; \quad(\alpha, \beta) \mapsto\langle\alpha, \beta\rangle:=\operatorname{pr}_{!}(\alpha \beta) .
$$

Proposition 9.5. The pairing $\langle\cdot, \cdot\rangle$ defined in (9.5) is non-degenerate and $\mathfrak{S}_{n}$-invariant.

Proof. We begin with $\mathfrak{S}_{n}$-invariance. Let $\alpha \in H^{2 k}(\operatorname{Hess}(\mathrm{S}, h))$ and $\beta \in H^{2 d-2 k}(\operatorname{Hess}(\mathrm{S}, h))$ and $v \in \mathfrak{S}_{n}$. Then

$$
\langle v \cdot \alpha, v \cdot \beta\rangle=\operatorname{pr}_{!}((v \cdot \alpha)(v \cdot \beta))=\operatorname{pr}_{!}(v \cdot(\alpha \beta))=v \cdot \operatorname{pr}_{!}(\alpha \beta)=\operatorname{pr}_{!}(\alpha \beta)=\langle\alpha, \beta\rangle
$$

where the second equality uses Lemma 9.2 , the third uses Lemma 9.4 and the fourth equality is because the $\mathfrak{S}_{n}$-action on $H^{*}(\mathrm{pt}) \cong \mathbb{Q}$ is trivial, as observed above.

Next we claim that the pairing $\langle\cdot, \cdot\rangle$ is non-degenerate. This is an elementary argument which is clearer when stated more generally. It is useful to recall that for a disconnected complex manifold $X=\sqcup_{a \in \mathcal{S}} X_{a}$ with connected components $X_{a}$ each of real dimension $2 d$, the cohomology ring $H^{*}(X)$ is a direct sum $\bigoplus_{a \in \mathcal{S}} H^{*}\left(X_{a}\right)$ (in particular, the cup product among different components vanishes) and the Gysin map is simply the sum of the individual Gysin maps associated to the projections $\operatorname{pr}_{a}: X_{a} \rightarrow$ pt, i.e. $\operatorname{pr}_{!}=$ $\sum_{a \in \mathcal{S}}\left(\operatorname{pr}_{a}\right)$ !. Thus it suffices to show that the given pairing is non-degenerate when restricted to the $a$-th component. But on each such component $X_{a}$, the Gysin map is given by capping with the fundamental homology class $\left[X_{a}\right] \in H_{2 d}\left(X_{a}\right)$ and the non-degeneracy becomes the usual statement of Poincare duality. Applying this argument to the case $X=\operatorname{Hess}(\mathrm{S}, h)$ yields the desired result.

Finally, we prove a fact which we use in the next section.

Lemma 9.6. $\operatorname{dim}_{\mathbb{Q}} H^{0}(\operatorname{Hess}(\mathrm{S}, h))^{\mathfrak{S}_{n}}=\operatorname{dim}_{\mathbb{Q}} H^{2 d}(\operatorname{Hess}(\mathrm{S}, h))^{\mathfrak{S}_{n}}=1$

Proof. We have seen in Proposition 9.5 that the pairing (9.5) is non-degenerate and $\mathfrak{S}_{n}$-invariant, so it follows that $H^{0}(\operatorname{Hess}(\mathrm{S}, h))$ and $H^{2 d}(\operatorname{Hess}(\mathrm{S}, h))$ are dual representations. This implies that $H^{0}(\operatorname{Hess}(\mathrm{S}, h))^{\mathfrak{S}_{n}} \cong$ $H^{2 d}(\operatorname{Hess}(\mathrm{S}, h))^{\mathfrak{S}_{n}}$. Now from the GKM description of $H_{T}^{*}(\operatorname{Hess}(\mathrm{S}, h))$ in (8.2) and the explicit formula for Tymoczko's $\mathfrak{S}_{n}$-action, it is not difficult to see directly that $H_{T}^{0}(\operatorname{Hess}(\mathrm{S}, h))^{\mathfrak{S}_{n}}$ is $\mathbb{Q}$-spanned by the identity element (whose component at each fixed point $w$ is 1 ); from this it also follows that $H^{0}(\operatorname{Hess}(\mathbf{S}, h))^{\mathfrak{S}_{n}}$ is $\mathbb{Q}$-spanned by the identity element, so $\operatorname{dim}_{\mathbb{Q}} H^{0}(\operatorname{Hess}(\mathrm{S}, h))^{\mathfrak{S}_{n}}=1$. By the above, this in turn implies $\operatorname{dim}_{\mathbb{Q}} H^{2 d}(\operatorname{Hess}(\mathrm{S}, h))^{\mathfrak{S}_{n}}=1$, as desired. 


\section{Proof of Theorem B}

In this section we prove Theorem B. As a first step, we prove the following.

Proposition 10.1. There exists a well-defined homomorphism of graded $\mathbb{Q}$-algebras $\mathcal{A}: H^{*}(\mathrm{Hess}(\mathrm{N}, h)) \rightarrow$ $H^{*}(\operatorname{Hess}(\mathrm{S}, h))$ making the diagram

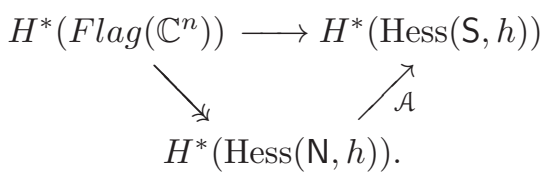

commute, where the other two maps are the induced homomorphisms. Moreover, the image of $\mathcal{A}$ lies in $H^{*}(\operatorname{Hess}(\mathrm{S}, h))^{\mathfrak{S}_{n}}$, and when the target is restricted to $H^{*}(\operatorname{Hess}(\mathrm{S}, h))^{\mathfrak{S}_{n}}$, then

$$
\mathcal{A}: H^{*}(\operatorname{Hess}(\mathrm{N}, h)) \rightarrow H^{*}(\operatorname{Hess}(\mathrm{S}, h))^{\mathfrak{S}_{n}}
$$

is surjective.

To prove Proposition 10.1 we (once again) first work with the equivariant cohomology $\operatorname{ring} H_{T}^{*}(\operatorname{Hess}(\mathrm{S}, h))$; we also capitalize on our explicit presentation of $H^{*}(\operatorname{Hess}(\mathrm{N}, h))$ obtained in Theorem A. More precisely, recall that the ordinary cohomology $\operatorname{ring} H^{*}\left(F \operatorname{lag}\left(\mathbb{C}^{n}\right)\right)$ is generated by the first Chern classes $\tau_{i}$ of the tautological line bundles $E_{i} / E_{i-1}$ as described in (2.11), and by Theorem A, the map $H^{*}\left(F \operatorname{lag}\left(\mathbb{C}^{n}\right)\right) \rightarrow H^{*}(\operatorname{Hess}(\mathrm{N}, h))$, induced by the inclusion $\operatorname{Hess}(\mathrm{N}, h) \hookrightarrow \operatorname{Flag}\left(\mathbb{C}^{n}\right)$, is surjective. Recall from (7.3) that

$$
\bar{\tau}_{i} \in H^{2}(\operatorname{Hess}(\mathrm{N}, h))
$$

is the image of $\tau_{i}\left(\operatorname{see}(2.10)\right.$ ). Then Theorem A shows that $H^{*}(\operatorname{Hess}(\mathbf{N}, h))$ is generated by the $\bar{\tau}_{i}$, and that the map sending the polynomial variable $x_{i}$ to $\bar{\tau}_{i}$ gives an isomorphism

$$
H^{*}(\operatorname{Hess}(\mathrm{N}, h)) \cong \mathbb{Q}\left[x_{1}, \ldots, x_{n}\right] /\left(\check{f}_{h(j), j}\left(x_{1}, \ldots, x_{n}\right) \mid 1 \leq j \leq n\right) .
$$

Now denote by

$$
\hat{\tau}_{i} \in H^{2}(\operatorname{Hess}(\mathrm{S}, h))
$$

the image of $\tau_{i}$ under the restriction map $H^{*}\left(F \operatorname{Fag}\left(\mathbb{C}^{n}\right)\right) \rightarrow H^{*}(\operatorname{Hess}(\mathrm{S}, h)$ ) (whereas the corresponding $T$ equivariant Chern class $\hat{\tau}_{i}^{T} \in H_{T}^{2}(\operatorname{Hess}(\mathrm{S}, h))$ was defined in (9.1)). That is, $\hat{\tau}_{i}$ is the first Chern class of the tautological line bundle over Flag $\left(\mathbb{C}^{n}\right)$ restricted to $\operatorname{Hess}(\mathrm{S}, h)$. In order to show that there exists a ring homomorphism $\mathcal{A}: H^{*}(\operatorname{Hess}(\mathrm{N}, h)) \rightarrow H^{*}(\operatorname{Hess}(\mathrm{S}, h))$ making (10.1) commute, from the above discussion it follows that it suffices to show that the images $\hat{\tau}_{i}$ of the $\tau_{i}$ in $H^{*}(\operatorname{Hess}(\mathrm{S}, h))$ also satisfy the relations specified by the $\left\{\check{f}_{h(j), j}\right\}_{1 \leq j \leq n}$, i.e. that

$$
\check{f}_{h(j), j}\left(\hat{\tau}_{1}, \ldots, \hat{\tau}_{n}\right)=0 \in H^{*}(\operatorname{Hess}(\mathrm{S}, h)) \text { for all } 1 \leq j \leq n .
$$

In order to prove (10.2), we will first work in the equivariant cohomology ring $H_{T}^{*}(\operatorname{Hess}(\mathrm{S}, h))$. Specifically, recall from (2.9) that $\tau_{i}^{T}$ is the $T$-equivariant first Chern class of the tautological line bundle $E_{i} / E_{i-1}$ in $H_{T}^{*}\left(F \operatorname{lag}\left(\mathbb{C}^{n}\right)\right)$, so that $\tau_{i}^{T}$ maps to $\tau_{i}$ under the forgetful map $H_{T}^{*}\left(F \operatorname{lag}\left(\mathbb{C}^{n}\right)\right) \rightarrow H^{*}\left(F \operatorname{lag}\left(\mathbb{C}^{n}\right)\right)$. Similarly $\hat{\tau}_{i}^{T}$ is the image of $\tau_{i}^{T}$ in $H_{T}^{*}(\operatorname{Hess}(\mathrm{S}, h))$ as defined in (9.1). Recall also that the kernel of the forgetful $\operatorname{map} H_{T}^{*}(\operatorname{Hess}(\mathrm{S}, h)) \rightarrow H^{*}(\operatorname{Hess}(\mathrm{S}, h))$ is the ideal $\left(t_{1}, \ldots, t_{n}\right) \subset H_{T}^{*}(\operatorname{Hess}(\mathrm{S}, h))$ generated by the classes $t_{i} \in H_{T}^{*}(\operatorname{Hess}(\mathrm{S}, h))$. Thus, in order to show the vanishing relations (10.2) it suffices to show that

$$
\check{f}_{h(j), j}\left(\hat{\tau}_{1}^{T}, \ldots, \hat{\tau}_{n}^{T}\right) \in\left(t_{1}, \ldots, t_{n}\right) \subset H_{T}^{*}(\operatorname{Hess}(\mathrm{S}, h)) \text { for all } 1 \leq j \leq n .
$$

This is precisely the goal of the next two lemmas.

We first define some classes in $H_{T}^{*}(\operatorname{Hess}(\mathrm{S}, h))$. Fix $j, k$ with $j, k \in[n]$. For each $w \in \mathfrak{S}_{n}$, we define a polynomial $g_{j, k}(w) \in \mathbb{Q}\left[t_{1}, \ldots, t_{n}\right]$ by

$$
g_{j, k}(w):= \begin{cases}\prod_{\ell=j+1}^{h(j)}\left(t_{k}-t_{w(\ell)}\right) & \text { if } k \in\{w(1), \ldots, w(j)\} \\ 0 & \text { otherwise, }\end{cases}
$$

where we take the convention $\prod_{\ell=j+1}^{j}\left(t_{k}-t_{w(\ell)}\right)=1$. Thus, for fixed $j$ and $k$, the collection $\left\{g_{j, k}(w)\right\}_{w \in \mathfrak{S}_{n}}$ specifies an element of $H_{T}^{*}\left(\operatorname{Hess}(\mathrm{S}, h)^{T}\right) \cong \bigoplus_{w \in \mathfrak{S}_{n}} \mathbb{Q}\left[t_{1}, \ldots, t_{n}\right]$. 
Lemma 10.2. The polynomials $\left\{g_{j, k}(w)\right\}_{w \in \mathfrak{S}_{n}}$ in (10.3) satisfy the GKM conditions (8.2) for Hess $(\mathrm{S}, h)$, and hence $g_{j, k}:=\left\{g_{j, k}(w)\right\}_{w \in \mathfrak{S}_{n}}$ is (the image under $\iota_{3}$ of) an equivariant cohomology class in $H_{T}^{*}(\mathrm{Hess}(\mathrm{S}, h)$ ).

Proof. Fix $j, k \in[n]$. For each $r \in[n]$, let us denote

$$
\mathfrak{S}_{n}^{r}:=\left\{w \in \mathfrak{S}_{n} \mid w(r)=k\right\}
$$

which is the set of permutations having $k$ at the $r$-th position in the one-line notation. Then we have a decomposition $\mathfrak{S}_{n}=\bigcup_{r=1}^{n} \mathfrak{S}_{n}^{r}$, and the condition $k \in\{w(1), \ldots, w(j)\}$ is equivalent to $w \in \bigcup_{r \leq j} \mathfrak{S}_{n}^{r}$. Recalling that the equivariant Chern class $\hat{\tau}_{i}^{T}$ satisfies $\hat{\tau}_{i}^{T}(w)=t_{w(i)}$ for $w \in \mathfrak{S}_{n}$ by (9.2), we can rewrite $g_{j, k}$ as

$$
g_{j, k}(w)= \begin{cases}\prod_{\ell=j+1}^{h(j)}\left(t_{k}-\hat{\tau}_{\ell}^{T}\right)(w) & \text { if } w \in \bigcup_{r \leq j} \mathfrak{S}_{n}^{r}, \\ 0 & \text { otherwise. }\end{cases}
$$

We now check that the collection $\left\{g_{j, k}(w)\right\}_{w \in \mathfrak{S}_{n}}$ satisfies the GKM condition (8.2) for Hess(S, $h$ ) by using (10.4). Let $w, w^{\prime} \in \mathfrak{S}_{n}$ with $w^{\prime}=w(a b)$ for some $a, b \in[n]$, and suppose that $w$ and $w^{\prime}$ are connected by an edge of the GKM graph of $\operatorname{Hess}(\mathrm{S}, h)$. We show that the difference $g_{j, k}(w)-g_{j, k}\left(w^{\prime}\right)$ is divisible by $t_{w(a)}-t_{w(b)}$ by taking cases.

Case 1. Suppose $w, w^{\prime} \in \bigcup_{r \leq j} \mathfrak{S}_{n}^{r}$. Note that the collection $\left\{\prod_{\ell=j+1}^{h(j)}\left(t_{k}-\hat{\tau}_{\ell}^{T}\right)(w)\right\}_{w \in \mathfrak{S}_{n}}$ satisfies the GKM condition for $\operatorname{Hess}(\mathrm{S}, h)$ since $\prod_{\ell=j+1}^{h(j)}\left(t_{k}-\hat{\tau}_{\ell}^{T}\right)$ is an element of $H_{T}^{*}(\operatorname{Hess}(\mathrm{S}, h))$ and we have the isomorphism (8.2). Thus the claim holds in this case by (10.4).

Case 2. Suppose $w, w^{\prime} \in \bigcup_{r>j} \mathfrak{S}_{n}^{r}$. In this case, the claim is immediate since $g_{j, k}(w)=g_{j, k}\left(w^{\prime}\right)=0$ by (10.4).

Case 3. Suppose $w \in \bigcup_{r \leq j} \mathfrak{S}_{n}^{r}$ and $w^{\prime} \in \bigcup_{r>j} \mathfrak{S}_{n}^{r}$. In this case, the condition $w^{\prime}=w(a b)$ implies that we have $w(a)=k$ or $w(b)=k$. Without loss of generality, we may assume that $w(a)=k$. This means $a \leq j$ because $w \in \bigcup_{r \leq j} \mathfrak{S}_{n}^{r}$. Similarly since we have $w^{\prime}(b)=k$ and $w^{\prime} \in \bigcup_{r>j} \mathfrak{S}_{n}^{r}$, it follows that $b>j$. Combining this with $a \leq j$, we obtain $a<b$. Hence the assumption that $w$ and $w^{\prime}$ are connected by an edge of the GKM graph of Hess $(\mathrm{S}, h)$ implies that $b \leq h(a)$. In particular, we obtain $j+1 \leq b \leq h(j)$ since $a \leq j$ implies $h(a) \leq h(j)$. Now from (10.4) we have

$$
g_{j, k}(w)-g_{j, k}\left(w^{\prime}\right)=\prod_{\ell=j+1}^{h(j)}\left(t_{k}-\hat{\tau}_{\ell}^{T}\right)(w)-0=\prod_{\ell=j+1}^{h(j)}\left(t_{k}-t_{w(\ell)}\right) .
$$

Since we have $w(a)=k$ and $j+1 \leq b \leq h(j)$ as discussed above, the above product contains $t_{k}-t_{w(b)}=$ $t_{w(a)}-t_{w(b)}$, and hence $g_{j, k}(w)-g_{j, k}\left(w^{\prime}\right)$ is divisible by $t_{w(a)}-t_{w(b)}$, as desired.

Next, we explicitly show (using the classes $g_{j, k}$ introduced above) that the classes $\check{f}_{h(j), j}\left(\hat{\tau}_{1}^{T}, \ldots, \hat{\tau}_{n}^{T}\right)$ are contained in the ideal of $H_{T}^{*}(\operatorname{Hess}(\mathrm{S}, h))$ generated by the $t_{i}$.

Lemma 10.3. Let $j \in[n]$. Then

$$
\check{f}_{h(j), j}\left(\hat{\tau}_{1}^{T}, \ldots, \hat{\tau}_{n}^{T}\right)=\sum_{k=1}^{n} t_{k} g_{j, k} \quad \text { in } H_{T}^{*}(\operatorname{Hess}(\mathrm{S}, h)) .
$$

In particular, $\check{f}_{h(j), j}\left(\hat{\tau}_{1}^{T}, \ldots, \hat{\tau}_{n}^{T}\right)$ lies in the ideal $\left(t_{1}, \ldots, t_{n}\right) \subset H_{T}^{*}(\operatorname{Hess}(\mathrm{S}, h))$ for all $j \in[n]$.

Proof. Since the restriction map $H_{T}^{*}(\operatorname{Hess}(\mathrm{S}, h)) \stackrel{\iota 3}{\rightarrow} H_{T}^{*}\left(\operatorname{Hess}(\mathrm{S}, h)^{T}\right)$ is injective, in order to prove the lemma it suffices to prove that for all $w \in \mathfrak{S}_{n}$ we have

$$
\check{f}_{h(j), j}\left(\hat{\tau}_{1}^{T}, \ldots, \hat{\tau}_{n}^{T}\right)(w)=\sum_{k=1}^{n} t_{k} g_{j, k}(w) \in \mathbb{Q}\left[t_{1}, \ldots, t_{n}\right] .
$$

Now recall that by definition, if $k \notin\{w(1), \ldots, w(j)\}$ then $g_{j, k}(w)=0$. Hence

$$
t_{k} g_{j, k}(w)= \begin{cases}t_{k} \prod_{\ell=j+1}^{h(j)}\left(t_{k}-t_{w(\ell)}\right) & \text { if } k \in\{w(1), \ldots, w(j)\} \\ 0 & \text { otherwise. }\end{cases}
$$


Thus if we take the sum of the $t_{k} g_{j, k}(w)$ over $k=1, \cdots, n$, it in fact suffices to take the sum only for $k=w(1), w(2), \ldots, w(j)$. Hence, we obtain by (6.3) that

$$
\sum_{k=1}^{n} t_{k} g_{j, k}(w)=\sum_{k=1}^{j}\left(t_{w(k)} \prod_{\ell=j+1}^{h(j)}\left(t_{w(k)}-t_{w(\ell)}\right)\right)=\check{f}_{h(j), j}(w)
$$

as desired.

From the above discussion and by Lemma 10.2 and Lemma 10.3. it is now clear that there exists a unique ring homomorphism

$$
\mathcal{A}: H^{*}(\operatorname{Hess}(\mathrm{N}, h)) \rightarrow H^{*}(\operatorname{Hess}(\mathrm{S}, h))
$$

which makes the diagram (1.6) in Theorem B commute. We note that $\mathcal{A}$ maps $\bar{\tau}_{i}$ to $\hat{\tau}_{i}$ for $i=1, \ldots, n$. We are now ready to prove Proposition 10.1

Proof of Proposition 10.1. First we claim that the image of $\mathcal{A}$ lies in the $\mathfrak{S}_{n}$-invariants. From the definition of $\mathcal{A}$, it is clear that its image coincides with the image of the restriction map $H^{*}\left(\operatorname{Flag}\left(\mathbb{C}^{n}\right)\right) \rightarrow H^{*}(\operatorname{Hess}(\mathrm{S}, h))$. Hence the claim follows from the facts that the $\mathfrak{S}_{n}$-representation on $H^{*}\left(F \operatorname{lag}\left(\mathbb{C}^{n}\right)\right)$ is trivial (Lemma 8.2) and that the bottom map in (8.1) is a homomorphism of $\mathfrak{S}_{n}$-representations. Now consider the map with restricted target

$$
\mathcal{A}: H^{*}(\operatorname{Hess}(\mathrm{N}, h)) \rightarrow H^{*}(\operatorname{Hess}(\mathrm{S}, h))^{\mathfrak{S}_{n}} .
$$

We wish to we show that this is surjective. Recalling the commutative diagram (10.1) and Lemma 8.2, it suffices to show that the map

$$
H^{*}\left(\operatorname{Flag}\left(\mathbb{C}^{n}\right)\right)^{\mathfrak{S}_{n}} \rightarrow H^{*}(\operatorname{Hess}(\mathrm{S}, h))^{\mathfrak{S}_{n}}
$$

is surjective. For this, we know from Proposition 9.3 that the map $H_{T}^{*}\left(\operatorname{Flag}\left(\mathbb{C}^{n}\right)\right)^{\mathfrak{S}_{n}} \rightarrow H_{T}^{*}(\operatorname{Hess}(\mathrm{S}, h))^{\mathfrak{S}_{n}}$ on equivariant cohomology is an isomorphism. We also know that the forgetful map $H_{T}^{*}(\operatorname{Hess}(\mathrm{S}, h)) \rightarrow$ $H^{*}(\operatorname{Hess}(\mathrm{S}, h))$ is surjective, and hence so is its restriction $H_{T}^{*}(\operatorname{Hess}(\mathrm{S}, h))^{\mathfrak{S}_{n}} \rightarrow H^{*}(\operatorname{Hess}(\mathrm{S}, h))^{\mathfrak{S}_{n}}$. Indeed, for any $\mathfrak{S}_{n}$-invariant element $x \in H^{*}(\operatorname{Hess}(\mathrm{S}, h))^{\mathfrak{S}_{n}}$ we can take a lift $\widetilde{x} \in H_{T}^{*}(\operatorname{Hess}(\mathrm{S}, h))$; averaging $\widetilde{x}$ over $\mathfrak{S}_{n}$ yields an $\mathfrak{S}_{n}$-invariant element which maps to $x$. Now, we see that (10.5) is surjective by taking $\mathfrak{S}_{n}$-invariant subrings in the commutative diagram in Lemma 8.1 completing the proof.

It remains to show that $\mathcal{A}: H^{*}(\operatorname{Hess}(\mathrm{N}, h)) \rightarrow H^{*}(\operatorname{Hess}(\mathrm{S}, h))^{\mathfrak{S}_{n}}$ is also injective (and hence an isomorphism). We achieve this by employing some basic commutative algebra facts concerning Poincaré duality algebras. The basic idea, encapsulated in Lemma 10.5 below, is the simple fact that if $\varphi: R \rightarrow S$ is a surjective graded algebra homomorphism from a Poincaré duality algebra and $\varphi$ induces an isomorphism between $R^{\max }$ and $S^{\max }$ (where $R^{\max }$ and $S^{\max }$ denote the highest-degree component of $R$ and $S$ respectively), then $\varphi$ must be an isomorphism. Since we have already shown above that $\mathcal{A}: H^{*}(\operatorname{Hess}(\mathrm{N}, h)) \rightarrow H^{*}(\operatorname{Hess}(\mathrm{S}, h))^{\mathfrak{S}_{n}}$ is surjective, Lemma 10.5 essentially reduces the question to showing that the domain is a Poincaré duality algebra and that $\mathcal{A}$ induces an isomorphism on the top degree.

There exist different definitions of Poincaré duality algebras in the literature, but we use the following.

Definition 10.4. Suppose that $R=\bigoplus_{i=0}^{d} R_{i}$ is a graded algebra over some fixed field $\mathfrak{k}$, finite-dimensional over $\mathfrak{k}$. Suppose $R_{0} \cong R_{d} \cong \mathfrak{k}$. We say $R$ is a Poincaré duality algebra (PDA) if the bilinear pairing $R_{i} \times R_{d-i} \rightarrow R_{d}$ defined by the multiplication in $R$ is non-degenerate for all $i=0, \ldots, d$.

The following straightforward lemma is the essence of our argument.

Lemma 10.5. Let $R=\bigoplus_{i=0}^{d} R_{i}$ and $R^{\prime}=\bigoplus_{i=0}^{d} R_{i}^{\prime}$ be graded algebras such that $R_{d} \neq\{0\}$ and $R_{d}^{\prime} \neq\{0\}$. Let $\varphi: R \rightarrow R^{\prime}$ be a graded ring homomorphism. Suppose that $R$ is a Poincaré duality algebra. If $\varphi$ is surjective and it restricts to an isomorphism between $R_{d}$ to $R_{d}^{\prime}$, then $\varphi$ is an isomorphism.

It remains to show that our rings $H^{*}(\operatorname{Hess}(\mathrm{N}, h))$ and $H^{*}(\operatorname{Hess}(\mathrm{S}, h))^{\mathfrak{S}_{n}}$ and the map $\mathcal{A}$ satisfies the conditions of Lemma 10.5. In particular, we wish to show that the $\operatorname{ring} H^{*}(\operatorname{Hess}(\mathrm{N}, h)) \cong \mathbb{Q}\left[x_{1}, \ldots, x_{n}\right] /\left(\check{f}_{h(j), j} \mid\right.$ $j \in[n])$ is a PDA. Recall that we showed in Lemma 6.8 that our generators $\breve{f}_{h(1), 1}, \check{f}_{h(2), 2}, \cdots, \breve{f}_{h(n), n}$ form a regular sequence of length equal to the number of variables in the polynomial ring. As is well-known in commutative algebra, these facts together imply that the cohomology ring $H^{*}(\operatorname{Hess}(\mathrm{N}, h))$ is a complete 
intersection, and in particular, is a PDA (see e.g. [32, Theorem 21.2.], 32, Theorem 21.3], and [24, Theorem 2.79]). We record this in the following proposition.

Proposition 10.6. Let $h \in H_{n}$ be a Hessenberg function and let $\operatorname{Hess}(\mathrm{N}, h)$ denote the associated regular nilpotent Hessenberg variety. Then, with respect to the usual grading and multiplication in cohomology, the ordinary cohomology ring $H^{*}(\operatorname{Hess}(\mathrm{N}, h))$ is a Poincaré duality algebra.

Now we can prove Theorem B.

Proof of Theorem B. We apply Lemma 10.5 to our $\mathbb{Q}$-algebra homomorphism $\mathcal{A}$ in (10.1). We already know that the map is surjective by Proposition 10.1 and that the domain of this map is Poincaré duality algebra from Proposition 10.6. Also, since we know that

$$
\operatorname{dim}_{\mathbb{Q}} H^{2 d}(\operatorname{Hess}(\mathrm{N}, h))=\operatorname{dim}_{\mathbb{Q}} H^{2 d}(\operatorname{Hess}(\mathrm{S}, h))^{\mathfrak{S}_{n}}=1
$$

from the computation of the Hilbert polynomial of $H^{*}(\operatorname{Hess}(\mathrm{N}, h))$ and Lemma 9.6, the surjectivity of $\mathcal{A}$ shows that the map $\mathcal{A}$ restricted on degree $2 d$ is an isomorphism. Hence, by Lemma 10.5 the $\mathbb{Q}$-algebra homomorphism $\mathcal{A}: H^{*}(\operatorname{Hess}(\mathrm{N}, h)) \rightarrow H^{*}(\operatorname{Hess}(\mathrm{S}, h))^{\mathfrak{S}_{n}}$ is an isomorphism, as desired.

\section{Connection to the Shareshian-Wachs conjecture}

As mentioned in the Introduction, our work on Hessenberg varieties turns out to be related to combinatorics through the Shareshian-Wachs conjecture. Although this conjecture has recently been proved by Brosnan and Chow, the approach taken in this paper offers a different perspective on the problem and, as we noted in the Introduction, our Theorem B proves (at least, for the coefficient of the Schur function $s_{n}(x)$ corresponding to the trivial representation) a statement which is strictly stronger than the corresponding statement in [8]. For this reason, in this section we briefly review the context, give the precise statement of the Shareshian-Wachs conjecture, and explain the relationship between the conjecture and our Theorem B.

In [40, 41, the Shareshian-Wachs conjecture is formulated in terms of natural unit interval orders and incomparability graphs, but for the purposes of this paper it is convenient to rephrase it more directly in terms of Hessenberg functions. Fix a Hessenberg function $h:[n] \rightarrow[n]$. Let $P(h)$ denote the partially ordered set whose underlying set is $[n]$ and with partial order defined by $j<_{P} i$ if and only if $h(j)<i$ [41, Section 4]. The following characterizes natural unit interval orders in terms of such posets.

Proposition 11.1. (41, Proposition 4.1]) Let $P$ be a poset on $[n]$. Then $P$ is a natural unit interval order if and only if $P=P(h)$ for some Hessenberg function $h$.

Furthermore, the incomparability graph of a poset $P$ as defined in [41, Section 1] has as its vertices the elements of $P$, and an edge between two elements precisely when the two elements are incomparable with respect to the given partial order. From the definition of $P(h)$ above, it is then immediate that the incomparability graph $G$ of $P(h)$ is the graph with vertex set $[n]$ and with edges $E$ given by

$$
E:=\{\{i, j\} \mid i, j \in[n], j<i \leq h(j)\}
$$

i.e. there is an edge between $i$ and $j$ (where without loss of generality $i>j$ ) exactly when $i \leq h(j)$. For example, if $h=(1,2, \ldots, n)$, then evidently $E$ is empty, and the corresponding incomparability graph $G$ has $n$ vertices and no edges. At the other extreme, if $h=(n, n, \ldots, n)$, then its comparability graph $G$ is the complete graph on $n$ vertices.

Next, let $x_{1}, x_{2}, x_{3}, \ldots$ be a countably infinite set of variables. Denoting by $\mathbb{P}$ the set of positive integers, we call a map $\kappa: V=[n] \rightarrow \mathbb{P}$ a coloring of $G$ if $\kappa$ satisfies $\kappa(i) \neq \kappa(j)$ for any $\{i, j\} \in E$, i.e. if $\kappa(i)$ is the "color" of the vertex $i$, then we require that adjacent vertices must be colored differently. Let $C(G)$ denote the set of all colorings of $G$, and let $\mathbf{x}_{\kappa}$ denote the monomial $\prod_{i \in[n]} x_{\kappa(i)}$ for any coloring $\kappa$. We also define

$$
\operatorname{asc}(\kappa):=\mid\{\{i, j\} \in E \mid j<i \text { and } \kappa(j)<\kappa(i)\} \mid
$$

Then the chromatic quasisymmetric function of $G$ is defined to be

$$
X_{G}(\mathbf{x}, t):=\sum_{\substack{\kappa \in C(G) \\ 36}} t^{\operatorname{asc}(\kappa)} \mathbf{x}_{\kappa} .
$$


In our situation, where $G=\operatorname{inc}(P(h))$ is the incomparability graph of a natural unit interval order $P(h)$, it is known that when we consider $X_{G}(\mathbf{x}, t)$ as a polynomial in $t$, each coefficient is an element of the algebra $\Lambda_{\mathbb{Z}}$ of symmetric functions in the variables $\mathbf{x}$ [41, Theorem 4.5]. That is, we have $X_{G}(\mathbf{x}, t) \in \Lambda_{\mathbb{Z}}[t]$. In the following example, for $i$ a positive integer, we denote by $e_{i}(\mathbf{x})$ the $i$-th elementary symmetric function in the variables $\mathbf{x}$.

Finally, following standard notation in the theory of symmetric functions, we denote by $\omega$ the involution of $\Lambda_{\mathbb{Z}}$, the algebra of symmetric functions, which exchanges the elementary basis $\left\{e_{\lambda}\right\}$ with the complete homogeneous basis $\left\{h_{\lambda}\right\}$ (as $\lambda$ ranges over partitions) [17, Section 6]. For our purposes it is useful to note that, for $\omega$ defined as above, we have $\omega\left(s_{\lambda}\right)=s_{\lambda^{*}}$, where $s_{\lambda}$ denotes the Schur function associated to a partition $\lambda$ [17, Section 6] and $\lambda^{*}$ denotes the partition conjugate to $\lambda$. Based on the above discussion, the reader may easily check that the formulation of the Shareshian-Wachs conjecture recorded below is equivalent to that given in [41, Conjecture 1.4].

Conjecture 11.2. Let $h:[n] \rightarrow[n]$ be a Hessenberg function, $P(h)$ its associated poset and $G$ the incomparability graph of $P(h)$. Let $X_{G}(\mathbf{x}, t)$ denote the chromatic quasisymmetric function of $G$, and let $\operatorname{Hess}(\mathrm{S}, h)$ be the regular semisimple Hessenberg variety associated to $h$. Then

$$
\omega X_{G}(\mathbf{x}, t)=\sum_{j=0}^{|E(G)|} \operatorname{ch} H^{2 j}(\operatorname{Hess}(\mathrm{S}, h)) t^{j}
$$

where ch denotes the Frobenius characteristic of Tymoczko's $\mathfrak{S}_{n}$-representation on $H^{2 j}(\operatorname{Hess}(\mathrm{S}, h))$.

Since (11.1) takes place within the ring of symmetric functions, expanding both sides in terms of (the basis of) Schur functions $s_{\lambda}(\mathbf{x})$, we may interpret (11.1) as the statement that the coefficient of $s_{\lambda}(\mathbf{x})$ on both sides must be equal for each partition $\lambda$. In [41, Theorem 6.9] Shareshian and Wachs also obtain a closed formula for the coefficient of $s_{n}(\mathbf{x})$, i.e. the coefficient corresponding to the trivial representation.

Theorem 11.3. ([41, Theorem 6.9]) In the Schur basis expansion of $X_{G}(\mathbf{x}, t)$, the coefficient of $s_{1^{n}}(\mathbf{x})$ is $\prod_{j=1}^{n}[h(j)-j+1]_{t}$ where $[i]_{t}=\frac{1-t^{i}}{1-t}$.

Finally, since $\omega s_{1^{n}}(\mathbf{x})=s_{n}(\mathbf{x})$ is the Frobenius characteristic of the trivial representation and the polynomial $\prod_{j=1}^{n}[h(j)-j+1]_{t}$ is exactly the Hilbert series $F\left(H^{*}(\operatorname{Hess}(\mathrm{N}, h)), s\right)$ by Lemma 6.4 (after replacing $s^{2}$ by $t$ ), it follows from Theorem B that Shareshian-Wachs conjecture holds for the component of the trivial representation. We record the following.

Corollary of Theorem B. The coefficients of $s_{n}(\mathbf{x})$ are the same on the both sides of (11.1).

\section{Appendix: Proof of Lemma 4.3}

The purpose of this section is to give a proof of Lemma 4.3 which we postponed to do that in Section 4 We divide the lemma into two claims, Lemma A.4 and Lemma A.5 below.

Lemma A.4. For any pair $k, j \in[n]$ with $k \geq j$, the polynomial $b_{k, j}$ is symmetric in the variables $u_{1}, \ldots, u_{j}$. Proof. We argue by induction on the indices $(k, j) \in[n]^{2}$ for $k \geq j$, with respect to the partial order defined by: $\left(k^{\prime}, j^{\prime}\right)<(k, j)$ if and only if $j^{\prime}<j$, or $j^{\prime}=j$ and $k^{\prime}<k$. Note also that the claim of the lemma clearly holds for any $b_{j, j}$ with $j \in[n]$, by its definition (4.5). Now we wish to show that the claim holds for $b_{k+1, j}$ for $k \geq j$ where we may assume by induction that the claim holds for $b_{k^{\prime}, j^{\prime}}$ with $\left(k^{\prime}, j^{\prime}\right)<(k+1, j)$. From the definition of $b_{k+1, j}$ in (4.6) it then follows that $b_{k+1, j}$ is symmetric in the first $j-1$ variables $u_{1}, \ldots, u_{j-1}$. Therefore, it now suffices to show that $b_{k+1, j}$ is also symmetric in $u_{j-1}$ and $u_{j}$.

For $k=j$ for any $j$, we may explicitly compute from (4.6) and (4.5) as follows:

$$
\begin{aligned}
b_{j+1, j}= & b_{j, j-1}-t b_{j-1, j-1}+u_{j}\left(b_{j, j}-b_{j-1, j-1}\right) \\
= & b_{j, j-1}-t b_{j-1, j-1}+u_{j}\left(u_{j}-(j-1) t\right) \\
= & \left(b_{j-1, j-2}-t b_{j-2, j-2}+u_{j-1}\left(u_{j-1}-(j-2) t\right)\right) \\
& \quad \quad-t\left(b_{j-2, j-2}+\left(u_{j-1}-(j-2) t\right)\right)+u_{j}\left(u_{j}-(j-1) t\right) \\
= & b_{j-1, j-2}-2 t b_{j-2, j-2}+\left(u_{j-1}^{2}+u_{j}^{2}-(j-1)\left(u_{j-1}+u_{j}\right) t+(j-2) t^{2}\right) .
\end{aligned}
$$


Since $b_{j-1, j-2}$ and $b_{j-2, j-2}$ are functions in the variables $u_{1}, \ldots, u_{j-2}$, it follows from the explicit expression above that $b_{j+1, j}=b_{j+1, j}\left(u_{1}, \ldots, u_{j}, t\right)$ is symmetric in $u_{j-1}$ and $u_{j}$.

We now claim $b_{k+1, j}$ is symmetric in $u_{j-1}$ and $u_{j}$ for $j \geq 2$ and $k>j$. Generalizing the argument for the case $k=j$ above, we may derive the following by repeated use of the inductive definition of the $b_{k, j}$ :

$$
\begin{aligned}
b_{k+1, j} & =b_{k, j-1}-t b_{k-1, j-1}+u_{j}\left(b_{k, j}-b_{k-1, j-1}\right) \\
& =b_{k, j-1}-t b_{k-1, j-1}-u_{j} t b_{k-2, j-1}+u_{j}^{2}\left(b_{k-1, j}-b_{k-2, j-1}\right) \\
& =\ldots \\
& =b_{k, j-1}-\sum_{q=j-1}^{k-1} u_{j}^{k-1-q} t b_{q, j-1}+u_{j}^{k+1-j}\left(b_{j, j}-b_{j-1, j-1}\right) \\
& =b_{k, j-1}-\sum_{q=j-1}^{k-1} u_{j}^{k-1-q} t b_{q, j-1}+u_{j}^{k+1-j}\left(u_{j}-(j-1) t\right) .
\end{aligned}
$$

Using this expression several times, we may express $b_{k+1, j}$ explicitly in terms of functions $b_{\ell, j-2}$ and the variables $u_{j-1}$ and $u_{j}$ :

$$
\begin{aligned}
b_{k+1, j}= & b_{k-1, j-2}-\sum_{r=j-2}^{k-2} u_{j-1}^{k-2-r} t b_{r, j-2}+u_{j-1}^{k+1-j}\left(u_{j-1}-(j-2) t\right) \\
& -u_{j}^{k-j} t b_{j-1, j-1} \\
& \quad-\sum_{q=j}^{k-1} u_{j}^{k-1-q} t\left(b_{q-1, j-2}-\sum_{r=j-2}^{q-2} u_{j-1}^{q-2-r} t b_{r, j-2}+u_{j-1}^{q+1-j}\left(u_{j-1}-(j-2) t\right)\right) \\
& +u_{j}^{k+1-j}\left(u_{j}-(j-1) t\right) \\
= & b_{k-1, j-2}-\sum_{r=j-2}^{k-2} u_{j-1}^{k-2-r} t b_{r, j-2}+u_{j-1}^{k+1-j}\left(u_{j-1}-(j-2) t\right) \\
& \quad-u_{j}^{k-j} t\left(b_{j-2, j-2}+\left(u_{j-1}-(j-2) t\right)\right) \\
& \quad-\sum_{q=j}^{k-1} u_{j}^{k-1-q} t\left(b_{q-1, j-2}-\sum_{r=j-2}^{q-2} u_{j-1}^{q-2-r} t b_{r, j-2}+u_{j-1}^{q+1-j}\left(u_{j-1}-(j-2) t\right)\right) \\
& +u_{j}^{k+1-j}\left(u_{j}-(j-1) t\right) .
\end{aligned}
$$

By exchanging the order of the sums with respect to $q$ and $r$, this is further equal to

$$
\begin{aligned}
& b_{k-1, j-2}-\sum_{r=j-2}^{k-2}\left(u_{j-1}^{k-2-r}+u_{j}^{k-2-r}\right) t b_{r, j-2}+\sum_{r=j-2}^{k-3}\left(\sum_{q=r+2}^{k-1} u_{j-1}^{q-(2+r)} u_{j}^{k-1-q}\right) t^{2} b_{r, j-2} \\
& \quad+\left(u_{j-1}^{k+2-j}+u_{j}^{k+2-j}\right)-(j-1)\left(u_{j-1}^{k+1-j}+u_{j}^{k+1-j}\right) t \\
& \quad+u_{j-1}^{k+1-j} t-\sum_{q=j-1}^{k-1}\left(u_{j-1}-(j-2) t\right) u_{j-1}^{q+1-j} u_{j}^{k-1-q} t .
\end{aligned}
$$

By separating the last summand, we obtain the equality

$$
\begin{aligned}
b_{k+1, j}=b_{k-1, j-2} & -\sum_{r=j-2}^{k-2}\left(u_{j-1}^{k-2-r}+u_{j}^{k-2-r}\right) t b_{r, j-2}+\sum_{r=j-2}^{k-3}\left(\sum_{q=r+2}^{k-1} u_{j-1}^{q-(2+r)} u_{j}^{(k-1)-q}\right) t^{2} b_{r, j-2} \\
+ & \left(u_{j-1}^{k+2-j}+u_{j}^{k+2-j}\right)-(j-1)\left(u_{j-1}^{k+1-j}+u_{j}^{k+1-j}\right) t \\
& +\sum_{q=j-1}^{k-1}(j-2) u_{j-1}^{q-(j-1)} u_{j}^{(k-1)-q} t^{2}-\sum_{q=j-1}^{k-2} u_{j-1}^{q-(j-1)+1} u_{j}^{(k-2)-q+1} t .
\end{aligned}
$$


Since $b_{k-1, j-2}$ and $b_{r, j-2}$ are functions in the variables $u_{1}, \ldots, u_{j-2}$, it can be seen from the final explicit expression above that $b_{k+1, j}=b_{k+1, j}\left(u_{1}, \ldots, u_{j}, t\right)$ is symmetric in the $u_{j-1}$ and $u_{j}$, as desired.

We now prove the second claim of Lemma 4.3. For this purpose, we make the substitution

$$
u_{r}=(w(r)-1) t \quad \text { for } r \in[n] .
$$

Lemma A.5. Let $k \geq j, k, j \in[n]$. Let $b_{k, j}=b_{k, j}((w(1)-1) t, \ldots) \in \mathbb{Q}[t]$ denote the polynomial $b_{k, j}$ defined in (4.5) and (4.6) evaluated at $u_{r}=(w(r)-1) t$ as in (A.2). Then for any pair $i, j \in[n]$ with $i \geq j$ we have

$$
f_{i, j}(w)=\sum_{k=j}^{i}(-1)^{i-k} e_{i-k}(w(j+1), \ldots, w(i)) t^{i-k} b_{k, j} \quad \text { in } \mathbb{Q}[t] .
$$

Proof. We prove the claim by induction on pairs $(i, j)$ with respect to the same partial order considered in the proof of Lemma A.4. First suppose $j=1$. In this case $b_{1,1}=u_{1}$ by definition and since $b_{*, 0}=0$ the equation (4.6) reduces to $b_{k+1,1}=u_{1} b_{k, 1}$, we obtain $b_{k, 1}=u_{1}^{k}$. Thus, for $j=1$ and any $i \geq j$, the claim (A.3) is precisely the assertion (4.4) obtained in Section 4 .

Now by induction suppose the equality A.3 holds for $j-1$ and for any $i \geq j-1$. Moreover, for $i=j$ we have $f_{j, j}(w)=b_{j, j}$ by definition of the $b_{j, j}$ so the assertion also holds in this case. Also for the case $i=j+1$ we can compute explicitly from (4.3) that the LHS of (A.3) is

$$
\begin{aligned}
f_{j+1, j}(w) & =f_{j, j-1}(w)+(w(j)-w(j+1)-1) t f_{j, j}(w) \\
& =\sum_{k=j-1}^{j}(-1)^{j-k} e_{j-k}(w(j)) t^{j-k} b_{k, j-1}+\left(u_{j}-w(j+1) t\right) b_{j, j} \\
& =-w(j) t b_{j-1, j-1}+b_{j, j-1}+\left(u_{j}-w(j+1) t\right) b_{j, j} \\
& =-\left(u_{j}+t\right) b_{j-1, j-1}+b_{j, j-1}+\left(u_{j}-w(j+1) t\right) b_{j, j}
\end{aligned}
$$

where we have used the inductive hypothesis, (A.2), and $f_{j, j}(w)=b_{j, j}$. The RHS of (A.3) can similarly be computed to be

$$
\begin{aligned}
\sum_{k=j}^{j+1}(-1)^{j+1-k} e_{j+1-k}(w(j+1)) t^{j+1-k} b_{k, j} & =-w(j+1) t b_{j, j}+b_{j+1, j} \\
& =-w(j+1) t b_{j, j}+\left(b_{j, j-1}+u_{j} b_{j, j}-\left(u_{j}+t\right) b_{j-1, j-1}\right) \\
& =-\left(u_{j}+t\right) b_{j-1, j-1}+b_{j, j-1}+\left(u_{j}-w(j+1) t\right) b_{j, j}
\end{aligned}
$$

where we have used (4.6). Comparing with the above, we may conclude that A.3 holds for $i=j+1$.

We now wish to show that A.3 holds for a pair $(i, j)$ with $i>j+1$, where we may also assume $j>1$. We will use the following facts and conventions concerning the elementary symmetric polynomials $e_{k}: e_{-1}=0$ and $e_{0}=1$ for any number of variables, and $e_{\ell}\left(y_{1}, \ldots, y_{s}\right)=0$ if $\ell>s$, i.e. if the expected degree is greater than the number of variables. With these conventions and from the definition of the elementary symmetric polynomials we may derive the identity

$$
e_{i-k-1}(w(j), \ldots, w(i-1))=e_{i-k-1}(w(j+1), \ldots, w(i-1))+w(j) e_{i-k-2}(w(j+1), \ldots, w(i-1))
$$

for any $k$ with $j-1 \leq k \leq i-1$. Now by the recursive description (4.3) of $f_{i, j}(w)$, the inductive hypotheses, (A.4), and (A.2), we can compute $f_{i, j}(w)$ to be

$$
\begin{aligned}
f_{i, j}(w)= & f_{i-1, j-1}(w)+\left(u_{j}-w(i) t\right) \cdot f_{i-1, j}(w) \text { by } \\
= & \sum_{k=j-1}^{i-1}(-1)^{i-k-1} e_{i-k-1}(w(j), \ldots, w(i-1)) t^{i-k-1} b_{k, j-1} \\
& \quad+\left(u_{j}-w(i) t\right) \cdot\left(\sum_{k=j}^{i-1}(-1)^{i-k-1} e_{i-k-1}(w(j+1), \ldots, w(i-1)) t^{i-k-1} b_{k, j}\right)
\end{aligned}
$$

by the inductive hypothesis 


$$
\begin{aligned}
=\sum_{k=j-1}^{i-1}( & -1)^{i-k-1}\left(e_{i-k-1}(w(j+1), \ldots, w(i-1))+w(j) e_{i-k-2}(w(j+1), \ldots, w(i-1))\right) t^{i-k-1} b_{k, j-1} \\
& \left.+\left(u_{j}-w(i) t\right) \cdot\left(\sum_{k=j}^{i-1}(-1)^{i-k-1} e_{i-k-1}(w(j+1), \ldots, w(i-1)) t^{i-k-1} b_{k, j}\right) \quad \text { by A.4 }\right) \\
=\sum_{k=j-1}^{i-1}(-1)^{i-k-1} e_{i-k-1}(w(j+1), \ldots, w(i-1)) t^{i-k-1} b_{k, j-1} & \\
& +\sum_{k=j-1}^{i-1}(-1)^{i-k-1} e_{i-k-2}(w(j+1), \ldots, w(i-1))\left(u_{j}+t\right) t^{i-k-2} b_{k, j-1} \\
& +\sum_{k=j}^{i-1}(-1)^{i-k-1} e_{i-k-1}(w(j+1), \ldots, w(i-1)) u_{j} t^{i-k-1} b_{k, j} \\
& \quad-\sum_{k=j}^{i-1}(-1)^{i-k-1} e_{i-k-1}(w(j+1), \ldots, w(i-1)) w(i) t^{i-k} b_{k, j} \quad \text { by (1.2.2). }
\end{aligned}
$$

By the convention that $e_{-1}=0, e_{\ell}\left(y_{1}, \ldots, y_{s}\right)=0$ if $\ell>s$ and by a re-indexing in order to gather terms, it follows that this is further equal to

$$
\begin{gathered}
\sum_{k=j-1}^{i-1}(-1)^{i-k-1} e_{i-k-1}(w(j+1), \ldots, w(i-1)) t^{i-k-1}\left(b_{k, j-1}+u_{j} b_{k, j}-\left(u_{j}+t\right) b_{k-1, j-1}\right) \\
-\sum_{k=j}^{i}(-1)^{i-k-1} e_{i-k-1}(w(j+1), \ldots, w(i-1)) w(i) t^{i-k} b_{k, j} .
\end{gathered}
$$

By the recursive definition (4.6) of $b_{k+1, j}$ for $k \geq j$, and because the term $e_{i-k-1}(w(j+1), \ldots, w(i-$ 1)) vanishes for $k=j-1$, we can replace the expressions $b_{k, j-1}+u_{j} b_{k, j}-\left(u_{j}+t\right) b_{k-1, j-1}$ in the first summand above with $b_{k+1, j}$. Then by re-indexing the first summand and using (a re-indexed version of) the equality (A.4), we obtain the following equality

$$
f_{i, j}(w)=\sum_{k=j}^{i}(-1)^{i-k} e_{i-k}(w(j+1), \ldots, w(i)) t^{i-k} b_{k, j} .
$$

as, desired. This proves the claim.

\section{REFERENCES}

[1] H. Abe, M. Harada, T. Horiguchi, and M. Masuda, The equivariant cohomology rings of regular nilpotent Hessenberg varieties in Lie type A: Research Announcement', Morfismos 18 (2014), No. 2, pp. 51-65.

[2] H. Abe, T. Horiguchi, The torus equivariant cohomology rings of Springer varieties, Topology Appl. 208 (2016), $143-159$.

[3] M. F. Atiyah and R. Bott, The moment map and equivariant cohomology, Topology 23 (1984), no. 1, 1-28.

[4] D. Anderson and J. Tymoczko, Schubert polynomials and classes of Hessenberg varieties, J. Algebra 323 (2010), no. 10, 2605-2623.

[5] D. Bayegan and M. Harada, Poset pinball, the dimension pair algorithm, and type A regular nilpotent Hessenberg varieties, ISRN Geometry, Article ID: 254235, 2012, doi:10.5402/2012/254235.

[6] N. Berline and M. Vergne, Classes caractéristiques équivariantes. Formule de localisation en cohomologie équivariante, C. R. Acad. Sci. Paris Sér. I Math. 295 (1982), no. 9, 539-541.

[7] M. Brion and J. Carrell, The equivariant cohomology ring of regular varieties, Michigan Math. J. 52 (2004), no. 1, $189-203$.

[8] P. Brosnan and T. Chow, Unit interval orders and the dot action on the cohomology of regular semisimple Hessenberg varieties, arXiv:1511.00773

[9] C. De Concini and C. Procesi, Symmetric functions, conjugacy classes and the flag variety, Invent. Math. 64 (1981), no. 2, 203-219.

[10] F. De Mari, On the topology of Hessenberg varieties of a matrix, Ph.D. thesis, Washington University, St. Louis, Missouri, 1987.

[11] F. De Mari, C. Procesi and M. A. Shayman, Hessenberg varieties, Trans. Amer. Math. Soc. 332 (1992), no. 2, 529-534. 
[12] F. De Mari and M. Shayman, Generalized Eulerian numbers and the topology of the Hessenberg variety of a matrix, Acta Appl. Math. 12 (1988), no. 3, 213-235.

[13] B. Dewitt and M. Harada, Poset pinball, highest forms, and ( $n-2,2)$ Springer varieties, Electron. J. Combin. 19 (2012), no. 1, Paper 56, 35 pp.

[14] E. Drellich, Combinatorics of equivariant cohomology: Flags and regular nilpotent Hessenberg varieties, PhD thesis, University of Massachusetts, 2015.

[15] Y. Fukukawa, M. Harada and M. Masuda, The equivariant cohomology rings of Peterson varieties, J. Math. Soc. Japan 67 (2015), no. 3, 1147-1159.

[16] J. Fulman, Descent identities, Hessenberg varieties, and the Weil Conjectures, J. Combin. Theory Ser. A 87 (1999), no. 2, 390-397.

[17] W. Fulton. Young Tableaux, London Mathematical Society Student Texts, 35. Cambridge University Press, Cambridge.

[18] F. Y. C. Fung. On the topology of components of some Springer fibers and their relation to Kazhdan-Lusztig theory, Adv. Math. 178 (2003), no. 2, 244-276.

[19] A. M. Garsia and C. Procesi, On certain graded $S_{n}$-modules and the q-Kostka polynomials, Adv. Math. 94 (1992), no. 1, $82-138$.

[20] M. Goresky, R. Kottwitz, and R. MacPherson, Equivariant cohomology, Koszul duality, and the localization theorem, Invent. Math. 131 (1998), no. 1, 25-83.

[21] M. Harada, T. Horiguchi and M. Masuda, The equivariant cohomology rings of Peterson varieties in all Lie types, Canad. Math. Bull. 58 (2015), no. 1, 80-90.

[22] M. Harada and J. Tymoczko, A positive Monk formula in the S1-equivariant cohomology of type A Peterson varieties, Proc. Lond. Math. Soc. (3) 103 (2011), no. 1, 40-72.

[23] M. Harada and J. Tymoczko, Poset pinball, GKM-compatible subspaces, and Hessenberg varieties, to appear in J. Math. Soc. Japan; arXiv:1007.2750

[24] T. Harima, T. Maeno, H. Morita, Y. Numata, A. Wachi and J. Watanabe, The Lefschetz properties, Lecture Notes in Mathematics, 2080. Springer, Heidelberg, 2013.

[25] T. Horiguchi, The $S^{1}$-equivariant cohomology rings of $(n-k, k)$ Springer varieties, Osaka J. Math, 52 (2015), no. 4, 1051-1063.

[26] W. Hsiang, Cohomology Theory of Topological Transformation Groups, Ergebnisse der Mathematik und ihrer Grenzgebiete, Band 85. Springer-Verlag, New York-Heidelberg, 1975.

[27] E. Insko and J. Tymoczko, Affine pavings of regular nilpotent Hessenberg varieties and intersection theory of the Peterson variety, arXiv:1309.0484

[28] E. Insko and A. Yong, Patch ideals and Peterson varieties, Transform. Groups 17 (2012), no. 4, 1011-1036.

[29] A. Klyachko, Orbits of a maximal torus on a flag space, Functional Anal. Appl. 19 (1985), no. 2, 65-66.

[30] A. Knutson, A Schubert calculus recurrence from the noncomplex $W$-action on $G / B$, arXiv:math/0306304.

[31] B. Kostant, Flag Manifold Quantum Cohomology, the Toda Lattice, and the Representation with Highest Weight $\rho$, Selecta Math. (N.S.) 2 (1996), no. 1, 43-91.

[32] H. Matsumura, Commutative ring theory, second edition, Cambridge Studies in Advanced Mathematics, 8, Cambridge University Press, Cambridge, 1989.

[33] A. Mbirika, A Hessenberg generalization of the Garsia-Procesi basis for the cohomology ring of Springer varieties, Electron. J. Combin. 17 (2010), no. 1, Research Paper 153, 29 pp.

[34] A. Mbirika and J. Tymoczko, Generalizing Tanisaki's ideal via ideals of truncated symmetric functions, J. Algebraic Combin. 37 (2013), no. 1, 167-199.

[35] M. Mimura and H. Toda, Topology of Lie Groups. I, II. Translated from the 1978 Japanese edition by the authors. Translations of Mathematical Monographs, 91. American Mathematical Society, Providence, RI, 1991.

[36] M. Precup, Affine pavings of Hessenberg varieties for semisimple groups, Sel. Math. New Series 19 (2013), 903-922.

[37] M. Precup, The connectedness of Hessenberg varieties, J. Algebra 437 (2015), 34-43.

[38] C. Procesi, The toric variety associated to Weyl chambers, Mots, 153-161, Lang. Raison. Calc., Hermés, Paris, 1990.

[39] K. Rietsch, Totally positive Toeplitz matrices and quantum cohomology of partial flag varieties, J. Amer. Math. Soc. 16 (2003), no. 2, 363-392 (electronic).

[40] J. Shareshian and M. L. Wachs, Chromatic quasisymmetric functions and Hessenberg varieties, Configuration spaces, 433-460, CRM Series, 14, Ed. Norm., Pisa, 2012.

[41] J. Shareshian and M. L. Wachs, Chromatic quasisymmetric functions, Adv. Math. 295 (2016), 497-551.

[42] E. Sommers and J. Tymoczko, Exponents for B-stable ideals, Trans. Amer. Math. Soc. 358 (2006), no. 8, 3493-3509.

[43] T. A. Springer, Trigonometric sums, Green functions of finite groups and representations of Weyl groups, Invent. Math. 36 (1976), 173-207.

[44] R.P. Stanley, Log-concave and unimodal sequences in algebra, combinatorics, and geometry, Graph theory and its applications: East and West (Jinan, 1986), 500-535, Ann. New York Acad. Sci., 576, New York Acad. Sci., New York, 1989.

[45] R. P. Stanley, Combinatorics and Commutative Algebra, Second Edition 1996, Birkhäuser, Boston.

[46] T. Tanisaki, Defining ideals of the closures of the conjugacy classes and representations of the Weyl groups, Tôhoku Math. J. (2) 34 (1982), no. 4, 575-585.

[47] N. Teff, Representations on Hessenberg varieties and Young's rule, 23rd International Conference on Formal Power Series and Algebraic Combinatorics (FPSAC 2011), 903-914, Discrete Math. Theor. Comput. Sci. Proc., AO, Assoc. Discrete Math. Theor. Comput. Sci., Nancy, 2011. 
[48] J. Tymoczko, Linear conditions imposed on flag varieties, Amer. J. Math. 128 (2006), no. 6, 1587-1604.

[49] J. Tymoczko, Permutation actions on equivariant cohomology of flag varieties, Toric topology, 365-384, Contemp. Math., 460, Amer. Math. Soc., Providence, RI, 2008.

Osaka City University Advanced Mathematical Institute, 3-3-138 Sugimoto, Sumiyoshi-ku, Osaka 558-8585, Japan / Department of Mathematics, University of Toronto, 40 St. George Street, Toronto, Ontario, Canada, M5S $2 \mathrm{E} 4$

E-mail address: hirakuabe@globe.ocn.ne.jp

Department of Mathematics and Statistics, McMaster University, 1280 Main Street West, Hamilton, Ontario L8S4K1, CANADA

E-mail address: Megumi.Harada@math.mcmaster.ca

$U R L:$ http://www .math.mcmaster.ca/ haradam

Osaka City University Advanced Mathematical Institute, 3-3-138 Sugimoto, Sumiyoshi-ku, Osaka 558-8585, Japan

E-mail address: tatsuya.horiguchi0103@gmail.com

Department of Mathematics, Osaka City University, 3-3-138 Sugimoto, Sumiyoshi-ku, Osaka 558-8585, Japan

E-mail address: masuda@sci.osaka-cu.ac.jp 